Supplementary Information for:

\title{
Late-Stage Modification of Electronic Properties of Antiaromatic and Diradicaloid Indeno[1,2-b]fluorene Analogues via Sulfur Oxidation
}

Justin J. Dressler, ${ }^{\dagger}$ Joshua E. Barker, ${ }^{\dagger}$ Hannah E. Hashimoto, ${ }^{\dagger}$ Lucas J. Karas, ${ }^{\dagger}$ Ryohei Kishi,,${ }^{\S}$ Lev N. Zakharov," Samantha N. MacMillan, ${ }^{\rrbracket}$ Carlos J. Gomez-Garcia, ${ }^{\perp}$ Masayoshi Nakano, ${ }^{\S}$ Judy I. Wu ${ }^{\ddagger}$ and Michael M. Haley*,†

$\dagger$ Department of Chemistry \& Biochemistry and the Materials Science Institute, University of Oregon, Eugene, Oregon 97403-1253, United States

$\$$ Department of Chemistry, University of Houston, Houston, Texas 77204, United States

$\S$ Department of Materials Engineering Science, Graduate School of Engineering Science, Osaka University, Toyonaka, Osaka 560-8531, Japan

"CAMCOR, University of Oregon, Eugene, Oregon 97403-1433, United States

${ }^{\pi}$ Department of Chemistry \& Chemical Biology, Cornell University, Ithaca, New York 14853, United States

${ }^{\perp}$ Department of Inorganic Chemistry and Instituto de Ciencia Molecular, Universidad de Valencia, 46980 Paterna, Spain

*email:haley@uoregon.edu

$\begin{array}{ll}\text { 1. Copies of NMR Spectra } & \text { S2 }\end{array}$

2. Additional Variable Temperature NMR Experiments $\quad$ S9

3. TD-DFT Calculations for UV-Vis Spectra $\quad$ S11

4. Additional Electronic Absorption Measurements $\quad$ S13

5. Cyclic Voltammetry $\quad$ S14

6. X-ray Diffraction $\quad$ S16

$\begin{array}{ll}\text { 7. Computational Details } & \text { S22 }\end{array}$

$\begin{array}{ll}\text { 8. SQUID Details and TGA Analysis } & \text { S50 }\end{array}$

9. References $\quad$ S51 


\section{Copies of NMR Spectra}

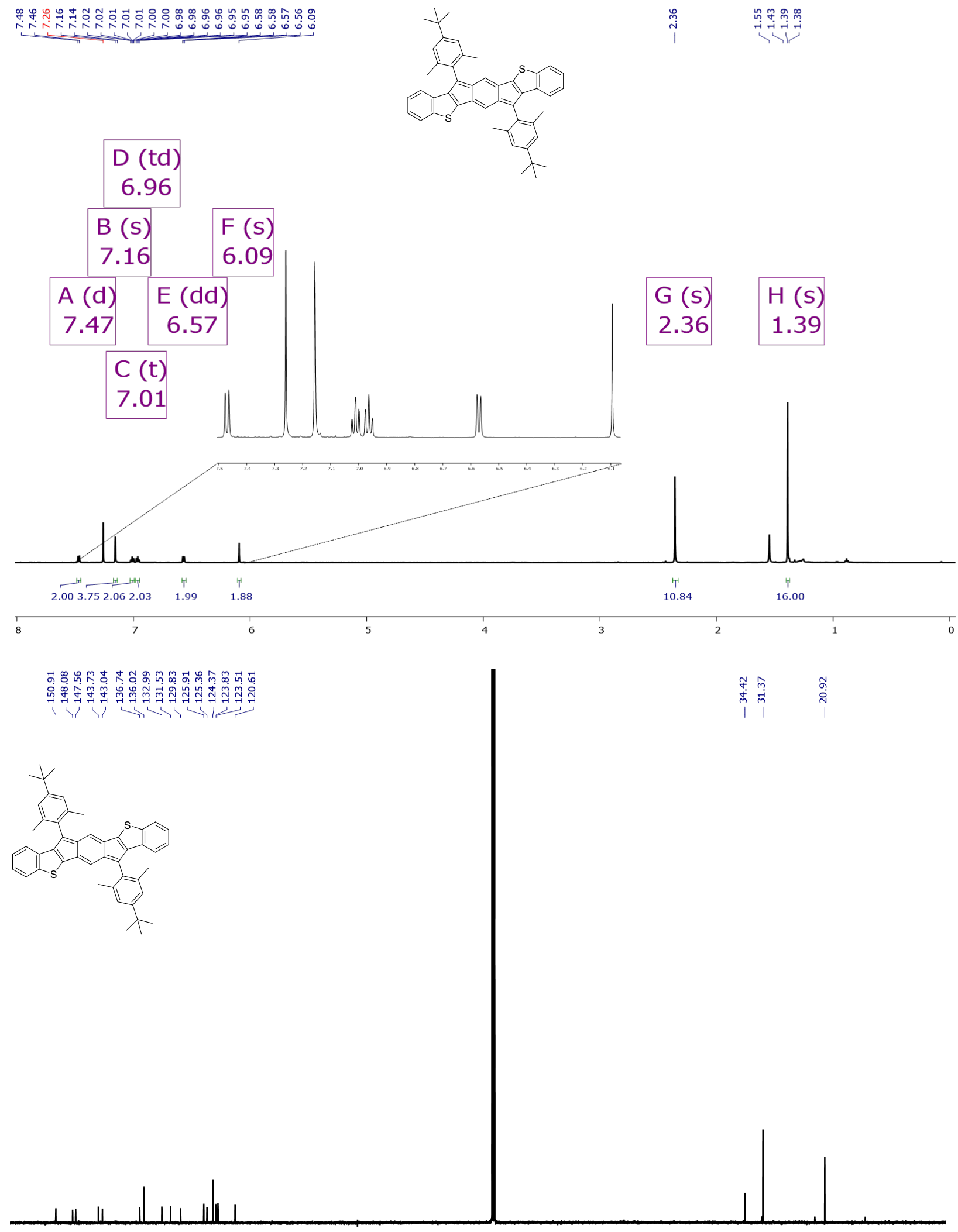

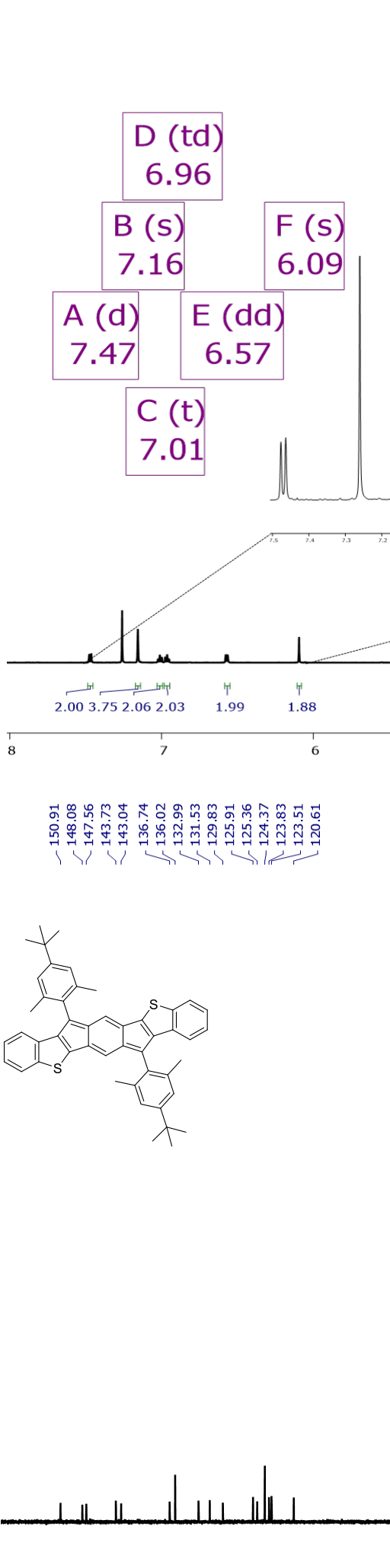




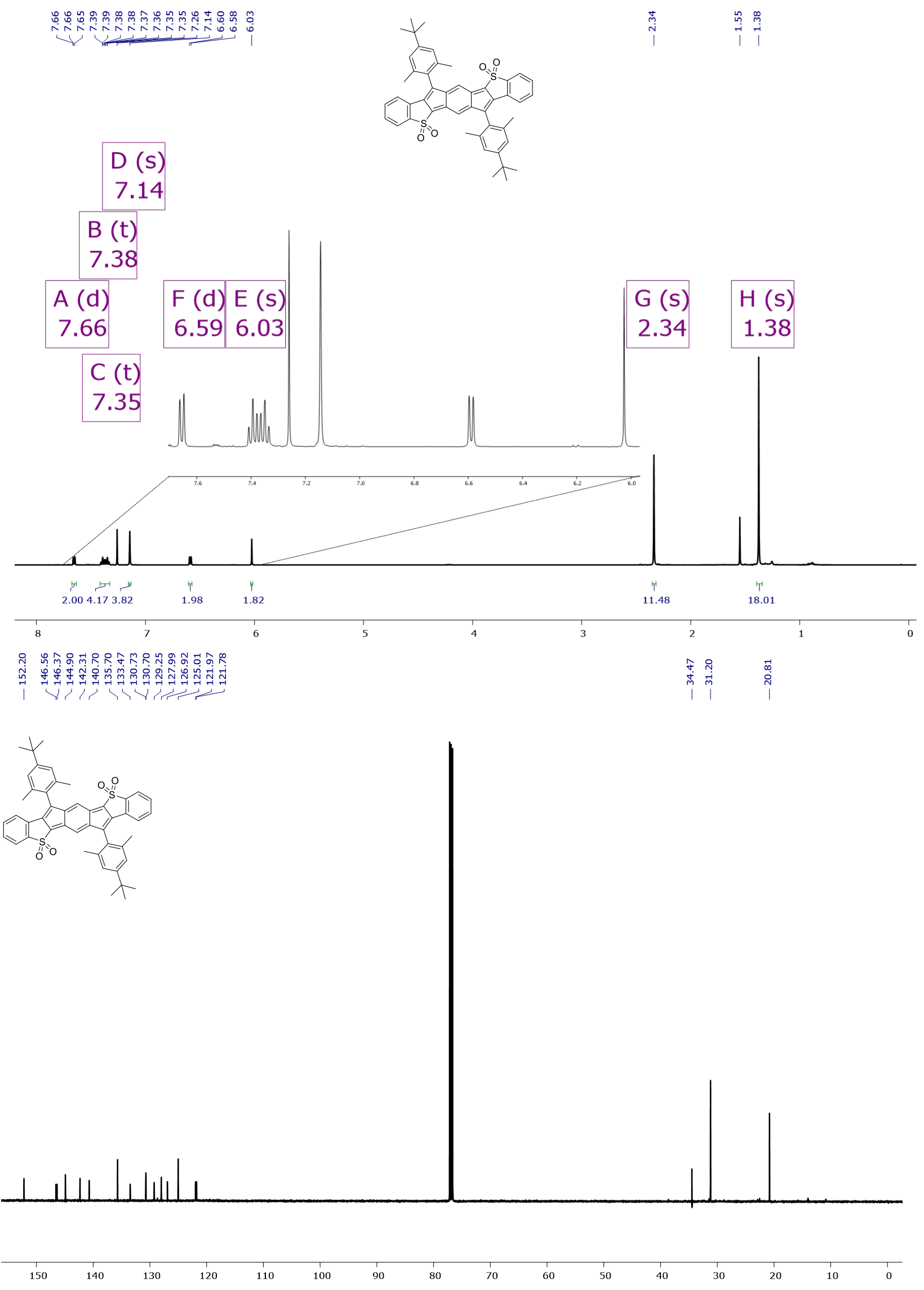



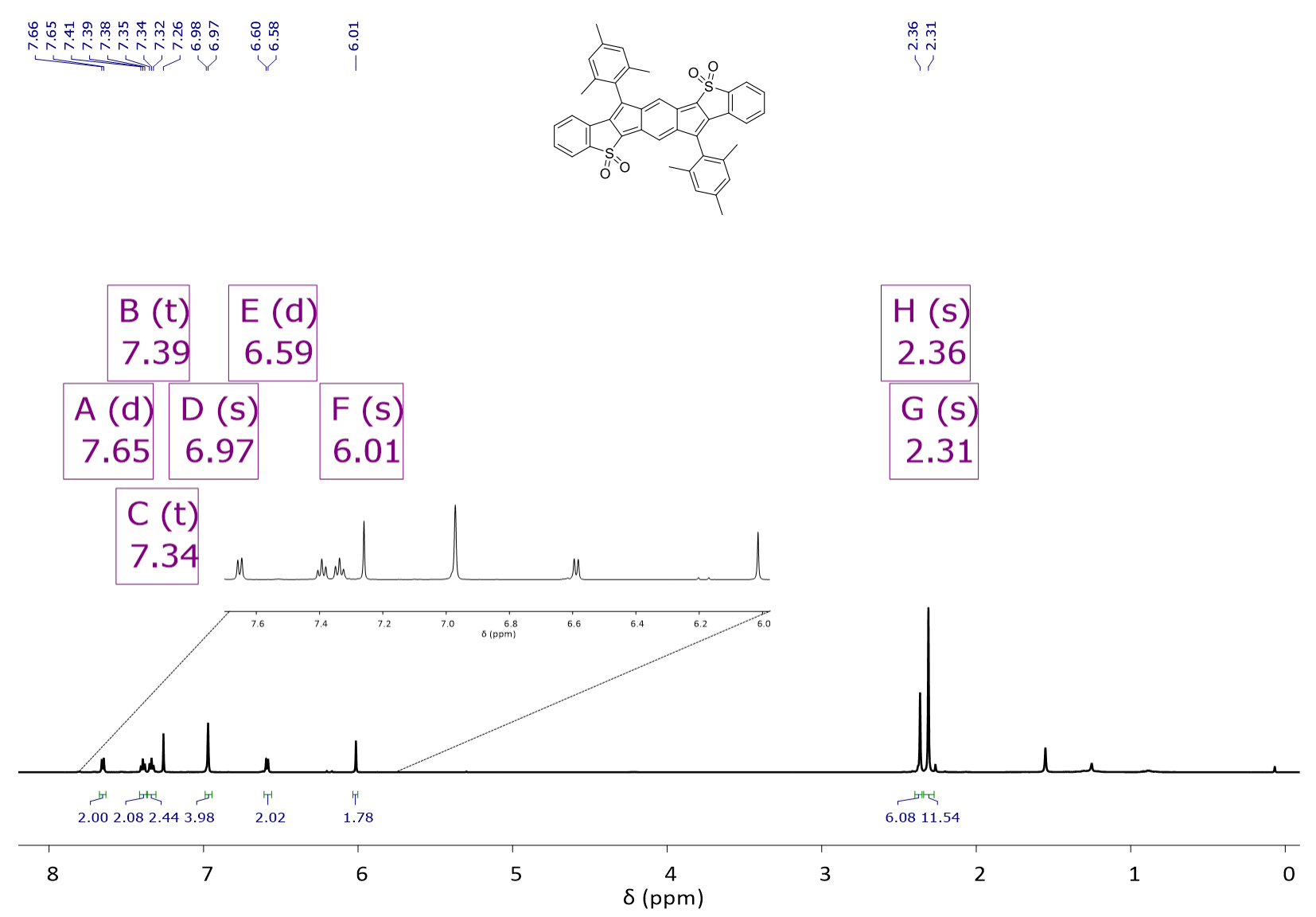

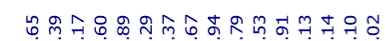

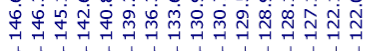
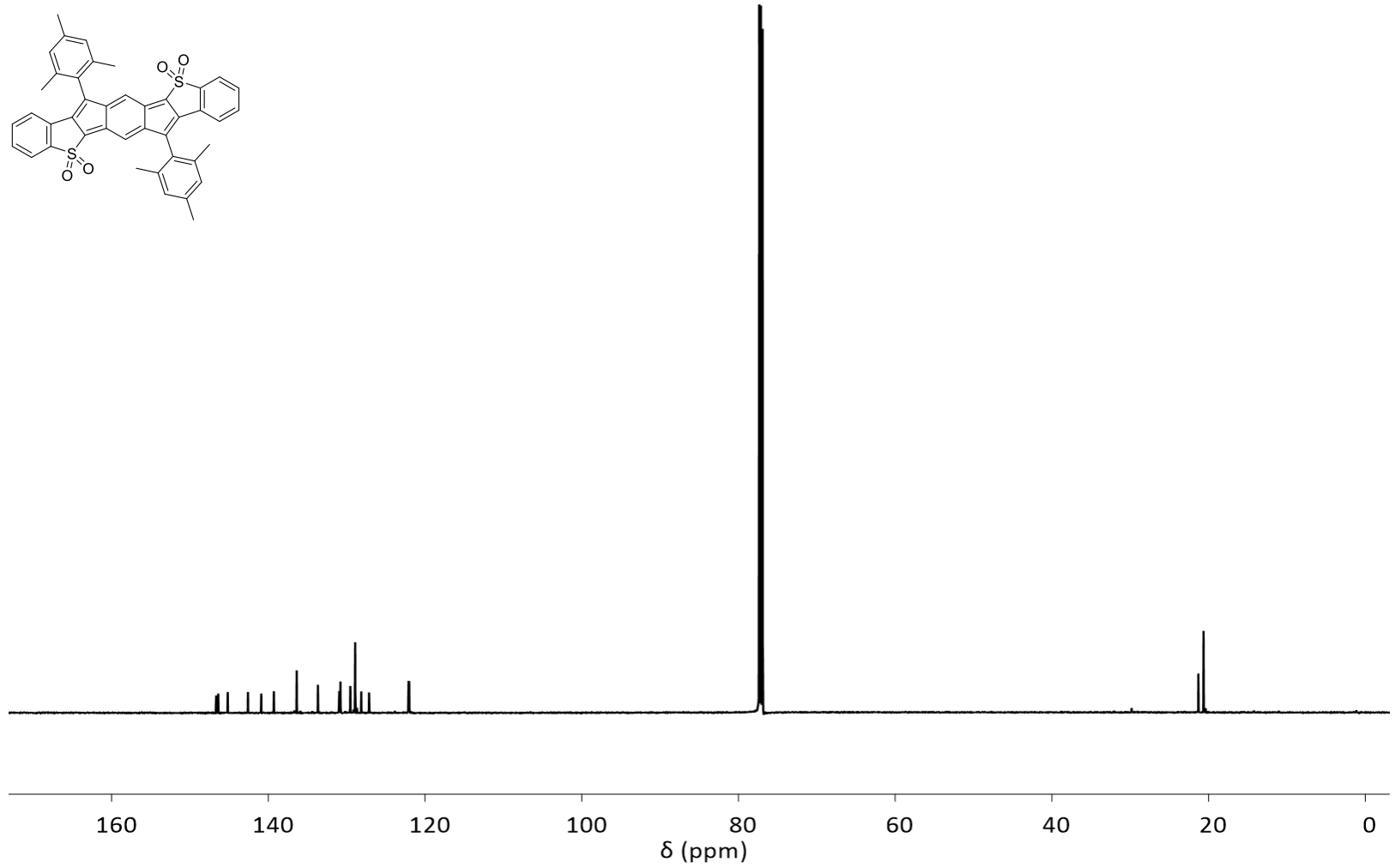


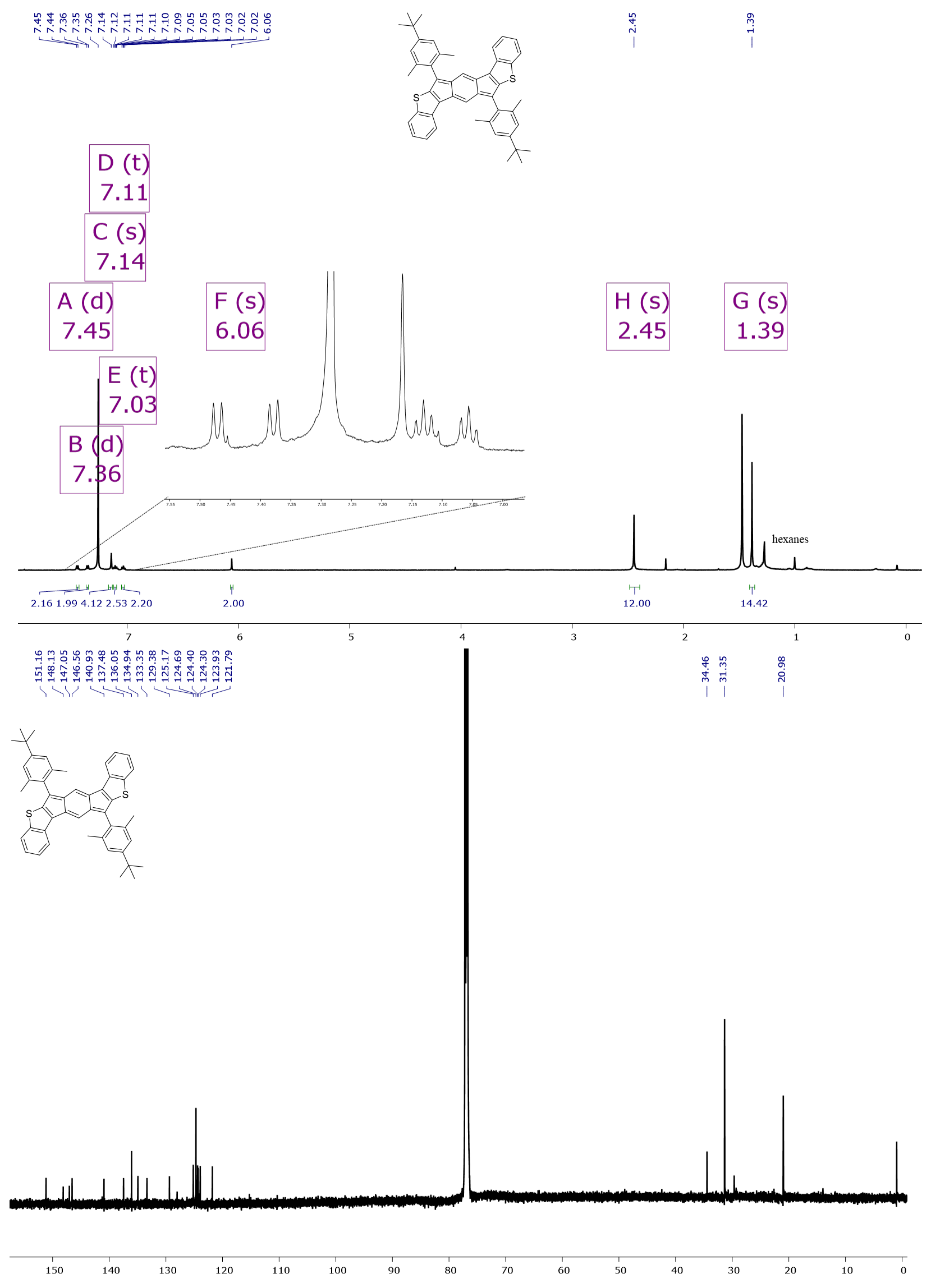


管

D (s)

7.17

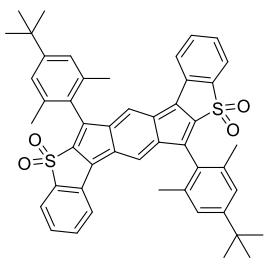

$$
\text { 운 }
$$

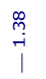

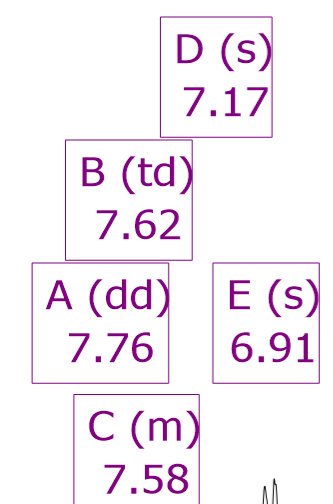

wholh
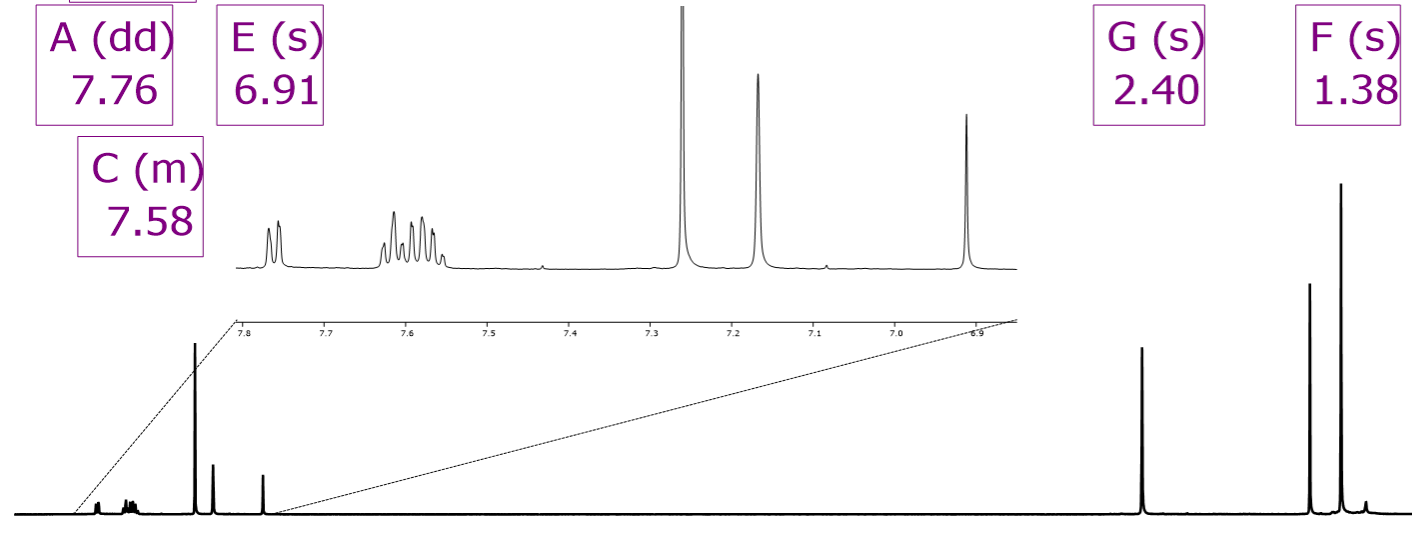

\begin{tabular}{l|l}
$2.40 \quad 1.38$
\end{tabular}

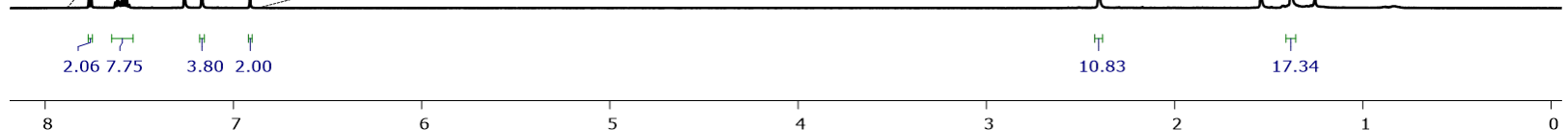

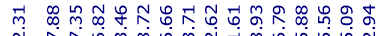

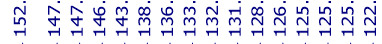
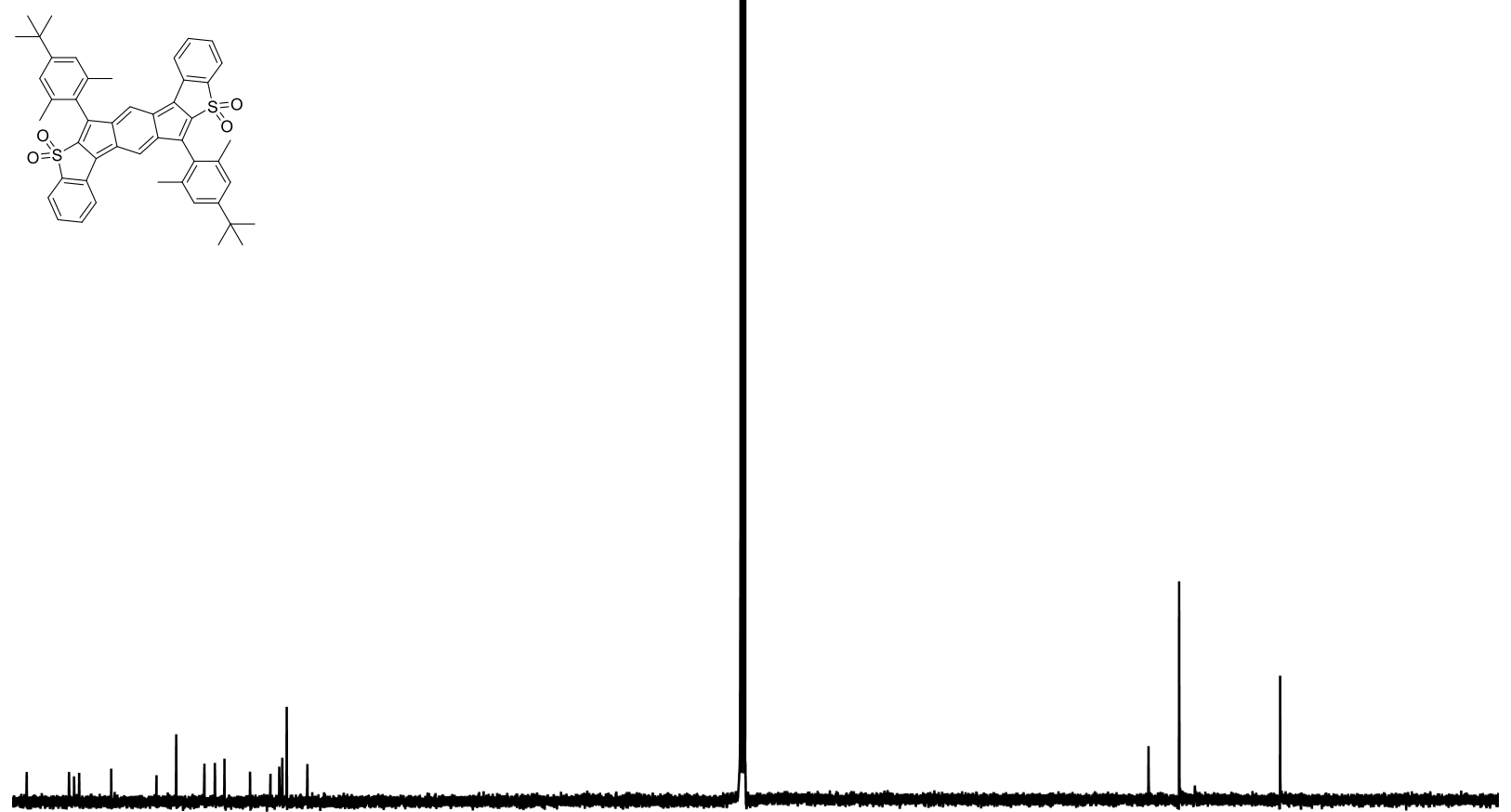

\begin{tabular}{|c|c|c|c|c|c|c|c|c|c|c|c|c|c|c|}
\hline 150 & 140 & 130 & 120 & 110 & 100 & 90 & 80 & 70 & 60 & 50 & 40 & 30 & 20 & 10 \\
\hline
\end{tabular}



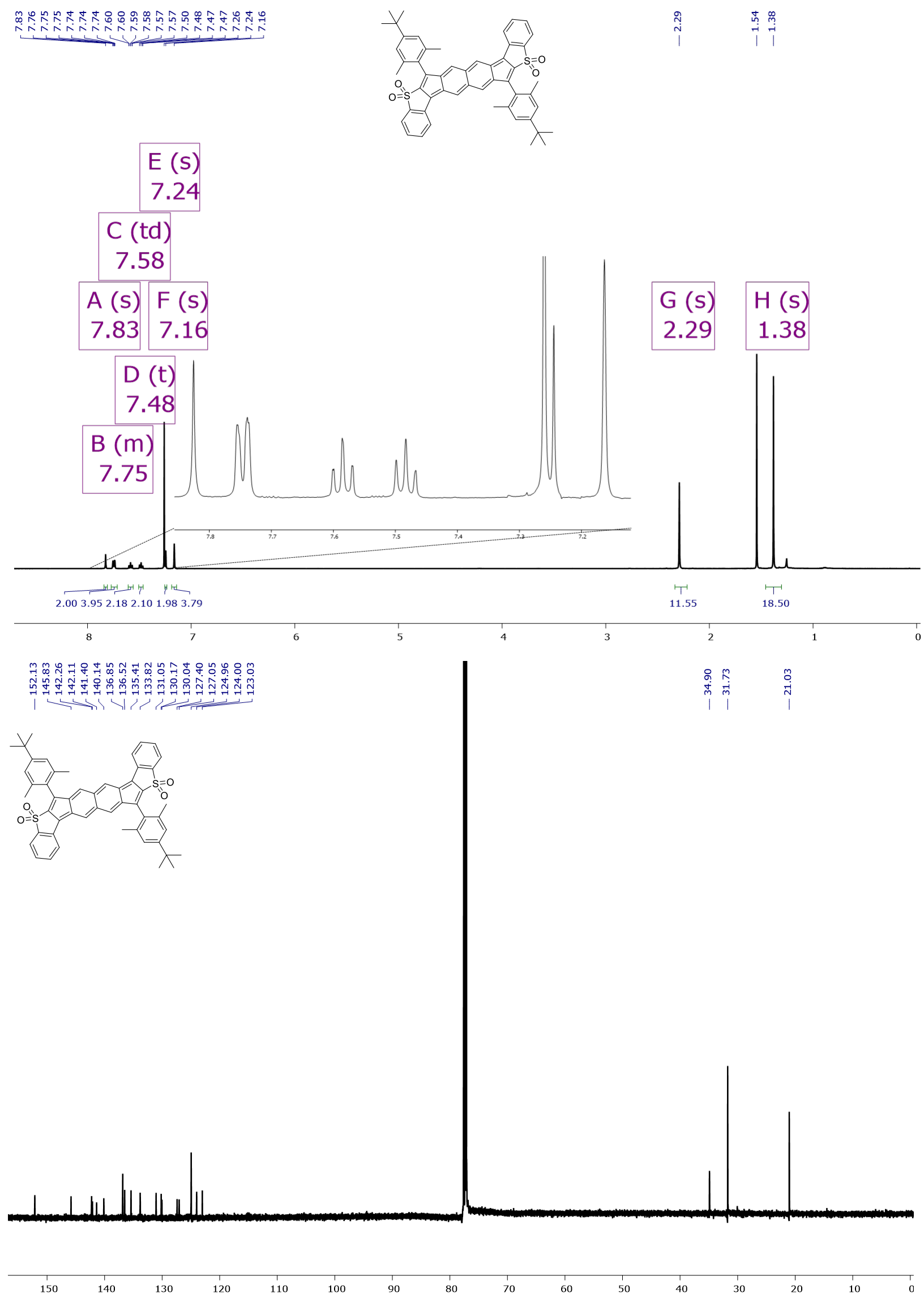


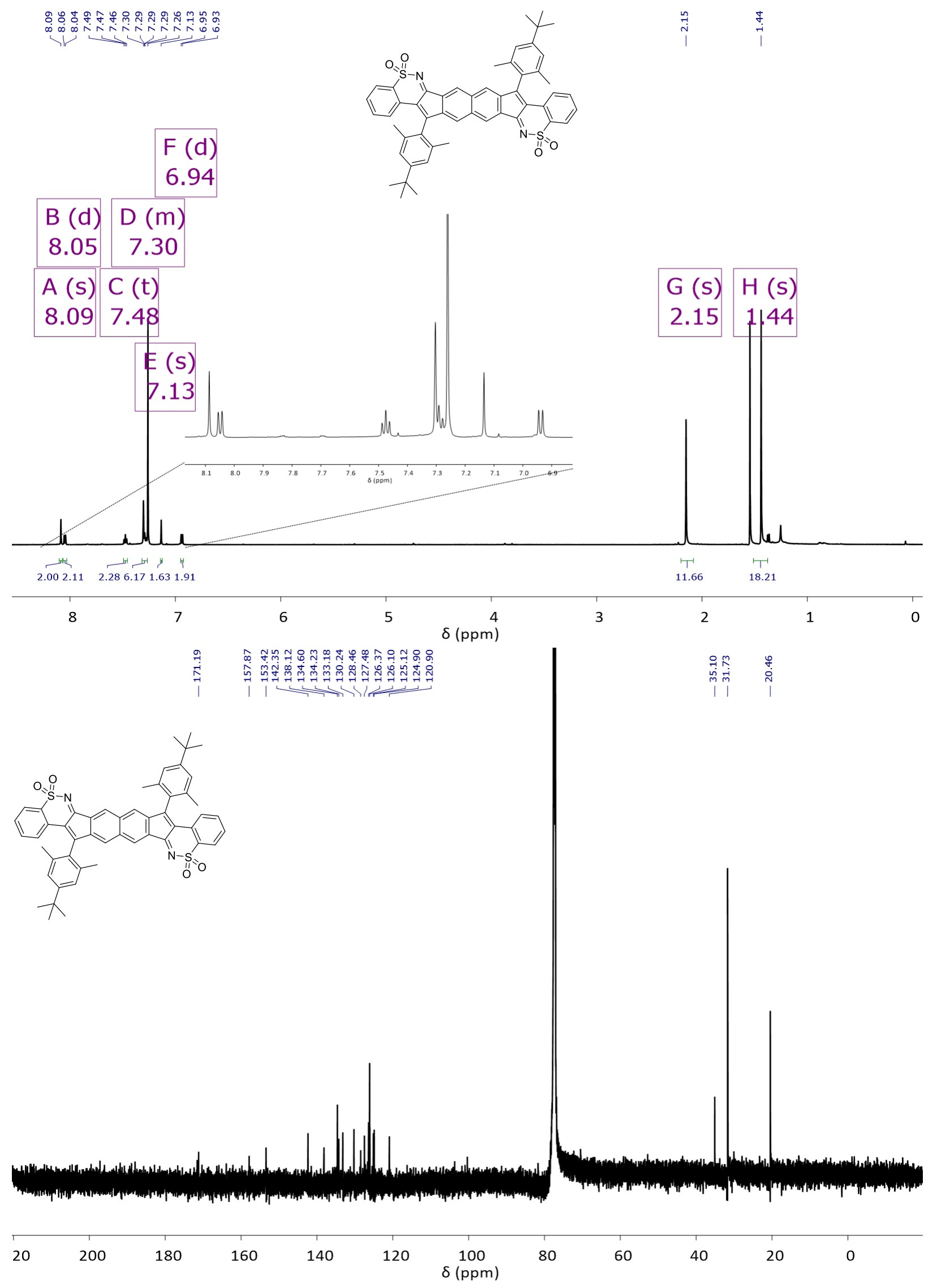




\section{Additional Variable Temperature NMR Experiments}

Approximately $5 \mathrm{mg}$ of $s y n$-IIDBT-sulfone (8) was placed in an NMR tube with different NMR solvents and the spectra were collected at different temperatures. The spectra were acquired on a Bruker $500 \mathrm{MHz}$ spectrometer that was heated to the maximum temperature range of each NMR solvent.

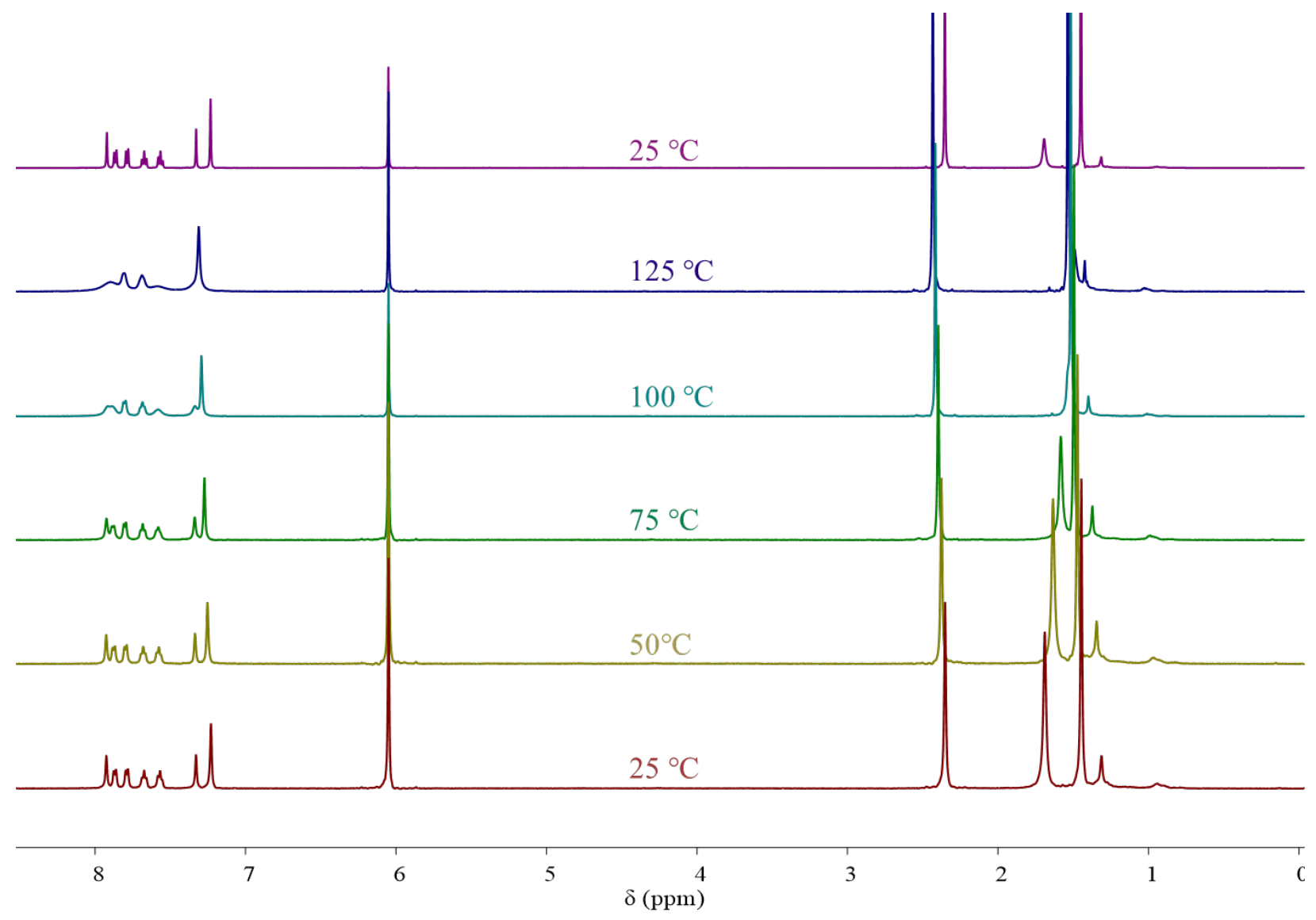

Figure S1. VT ${ }^{1} \mathrm{H}$ NMR full spectra of $\mathbf{8}$ in 1,1,2,2-tetrachloroethane- $d_{2}$ showing thermal population of the paramagnetic triplet state at elevated temperatures. 


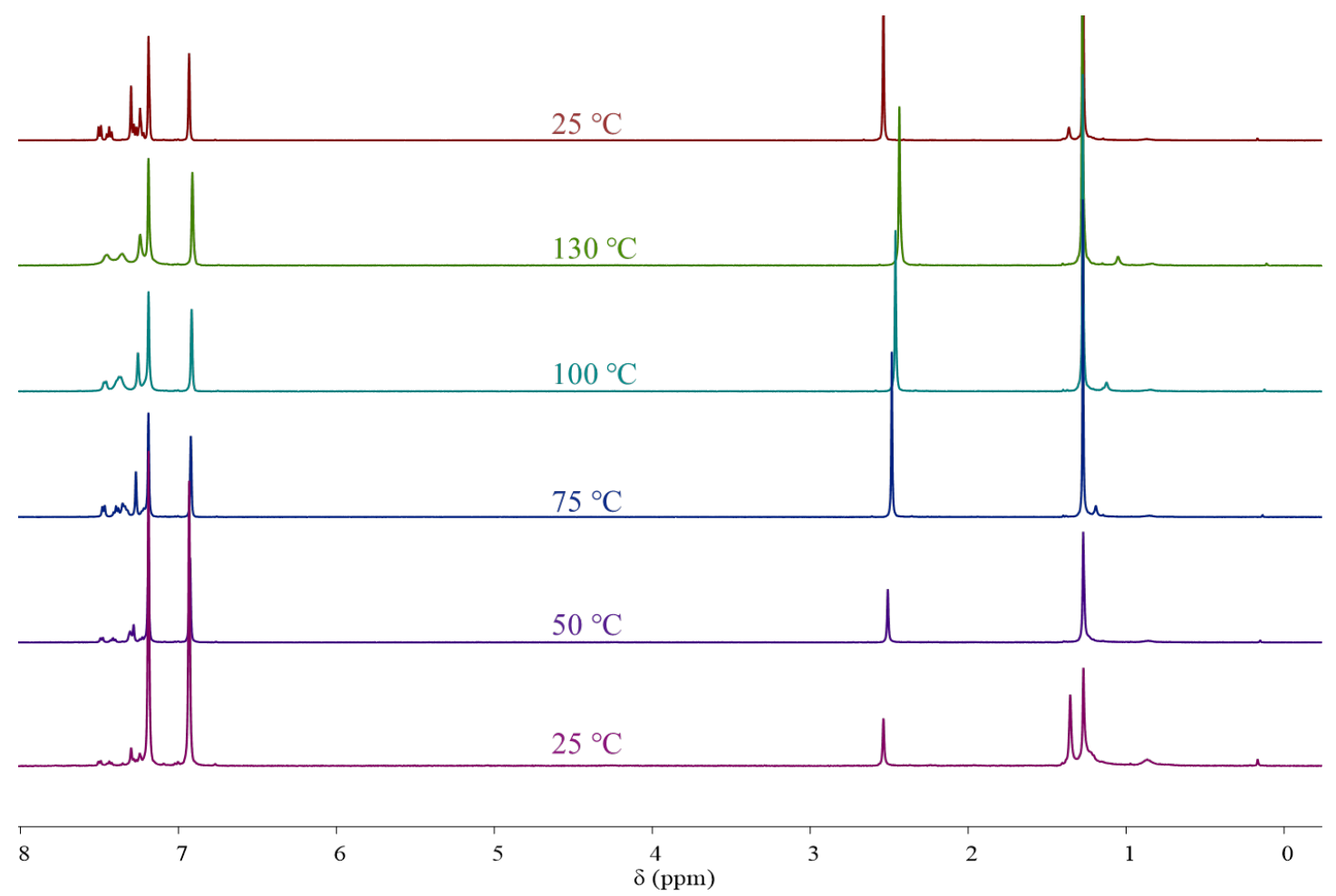

Figure S2. VT ${ }^{1} \mathrm{H}$ NMR full spectra of 8 in 1,2-dichlorobenzene- $d_{4}$ showing thermal population of the paramagnetic triplet state at elevated temperatures.

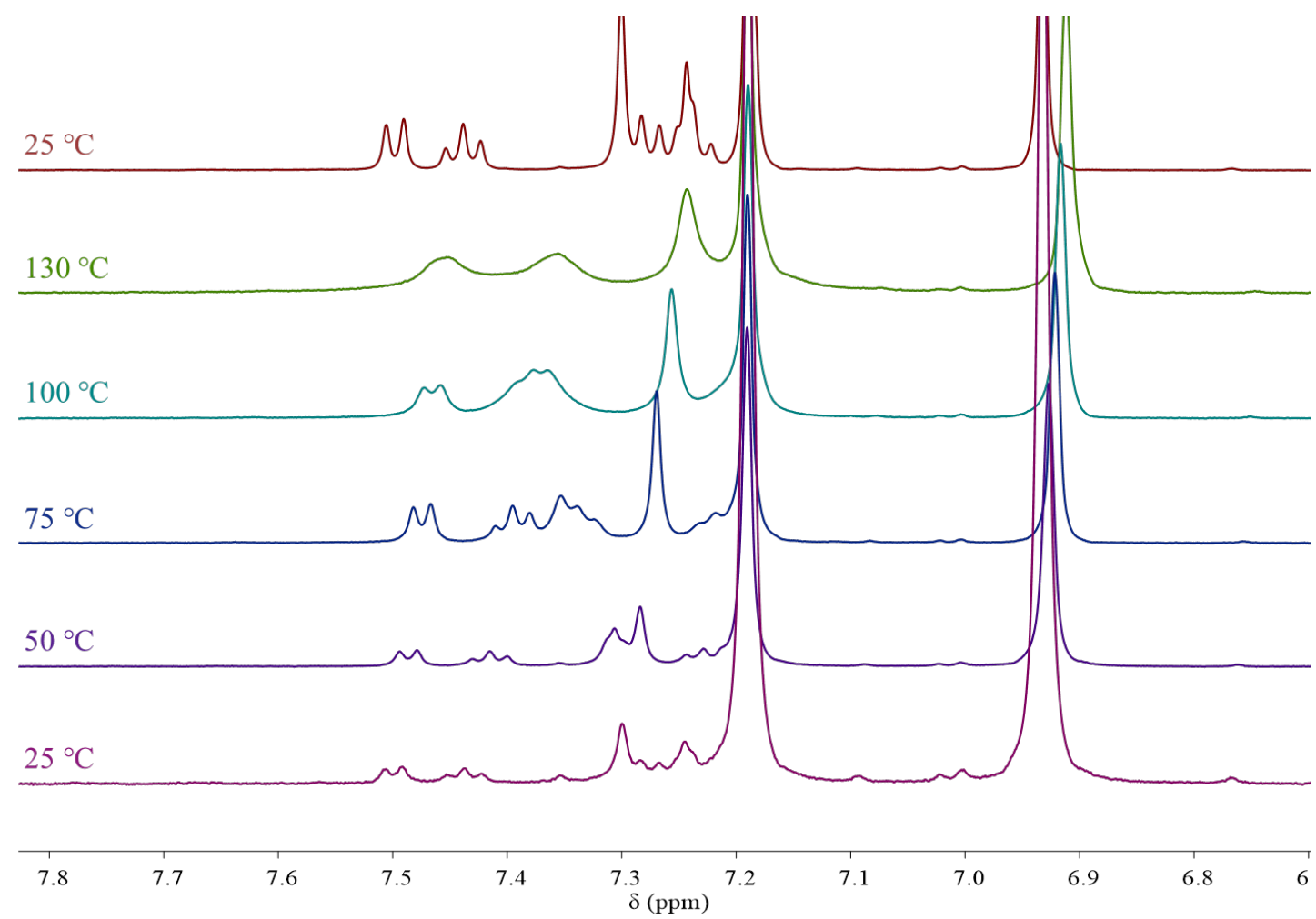

Figure S3. VT ${ }^{1} \mathrm{H}$ NMR of the aromatic region of $\mathbf{8}$ in 1,2-dichlorobenzene- $d_{4}$ showing thermal population of the paramagnetic triplet state at elevated temperatures. 


\section{TD-DFT Calculations for UV-Vis Spectra}
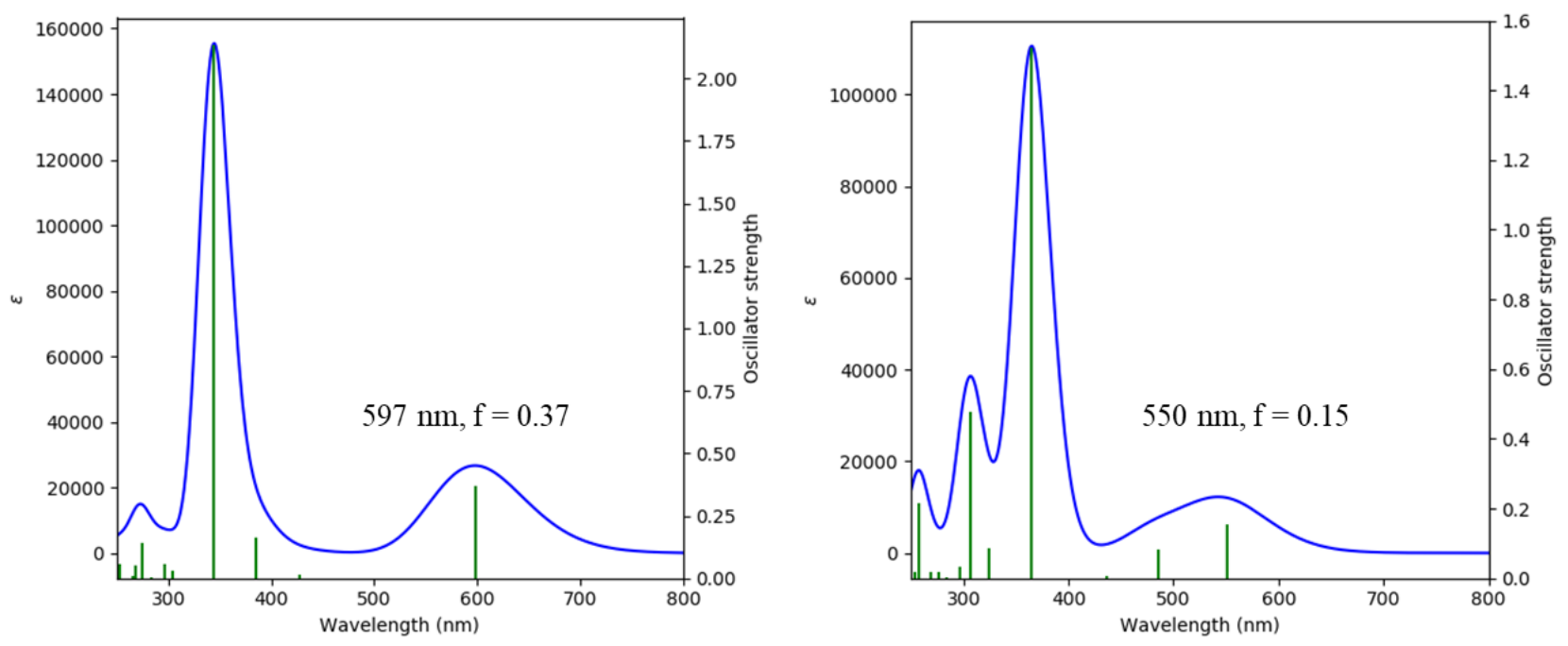

Figure S4. TD-DFT predicted UV-Vis spectra for (left) anti-IDBT (1) and for (right) anti-IDBTsulfone (5) calculated at the TD-B3LYP/6-311++G** level of theory.
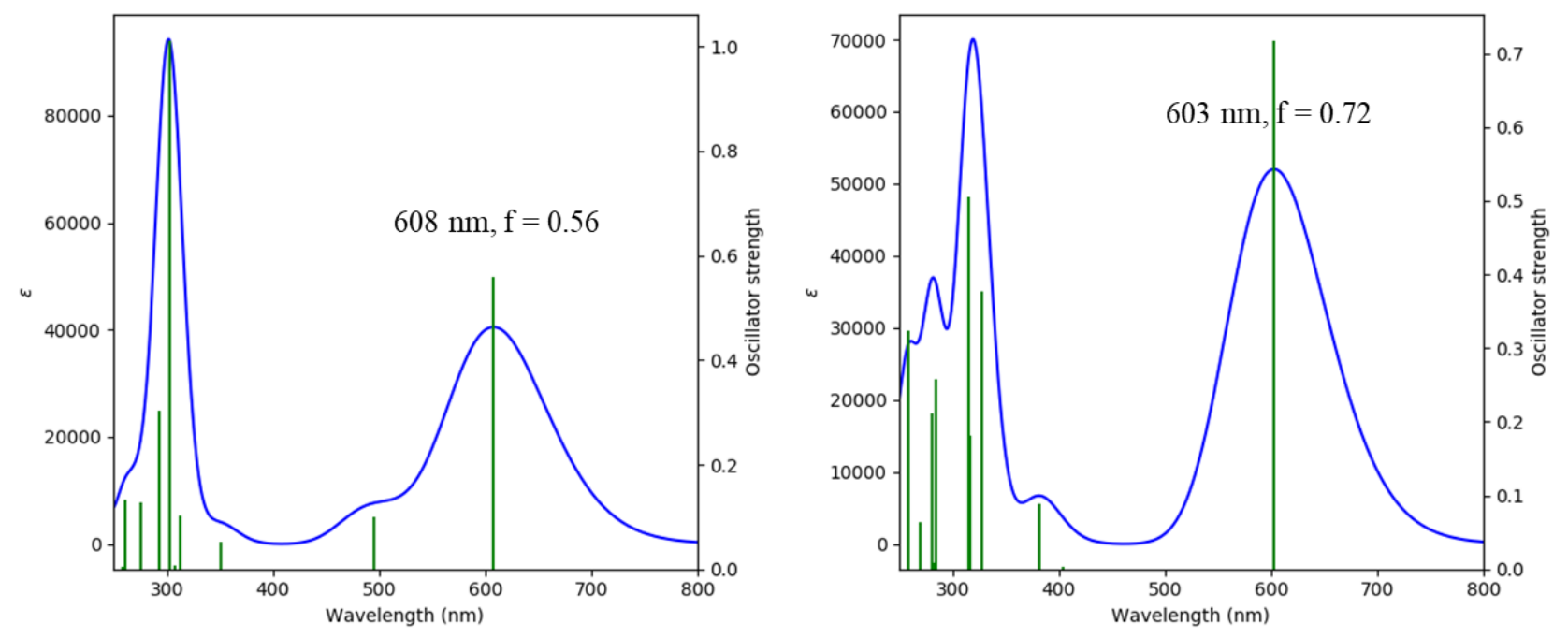

Figure S5. TD-DFT predicted UV-Vis spectra for (left) $s y n$-IDBT (2) and for (right) $s y n$-IDBTsulfone (6) calculated at the TD-B3LYP/6-311++G** level of theory. 

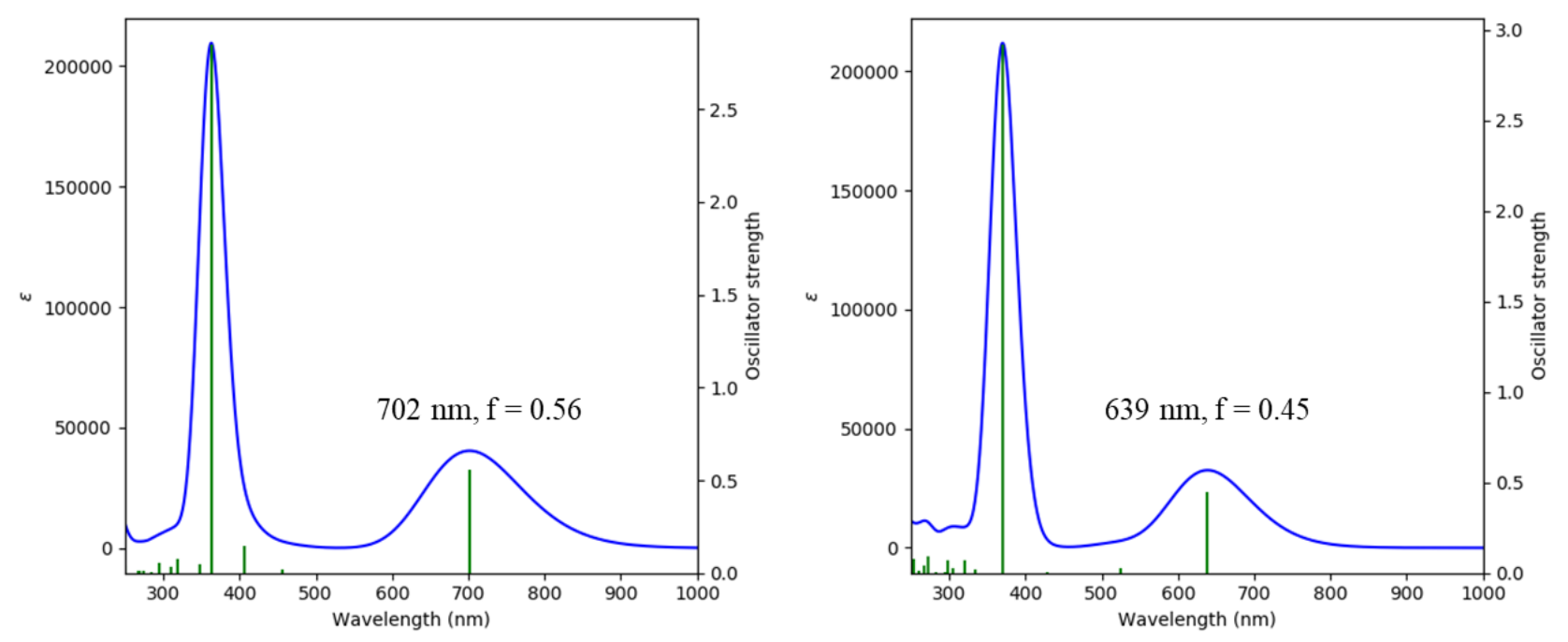

Figure S6. TD-DFT predicted UV-Vis spectra for (left) anti-IIDBT (3) and for (right) antiIIDBT-sulfone (7) calculated at the TD-B3LYP/6-311+G* level of theory.
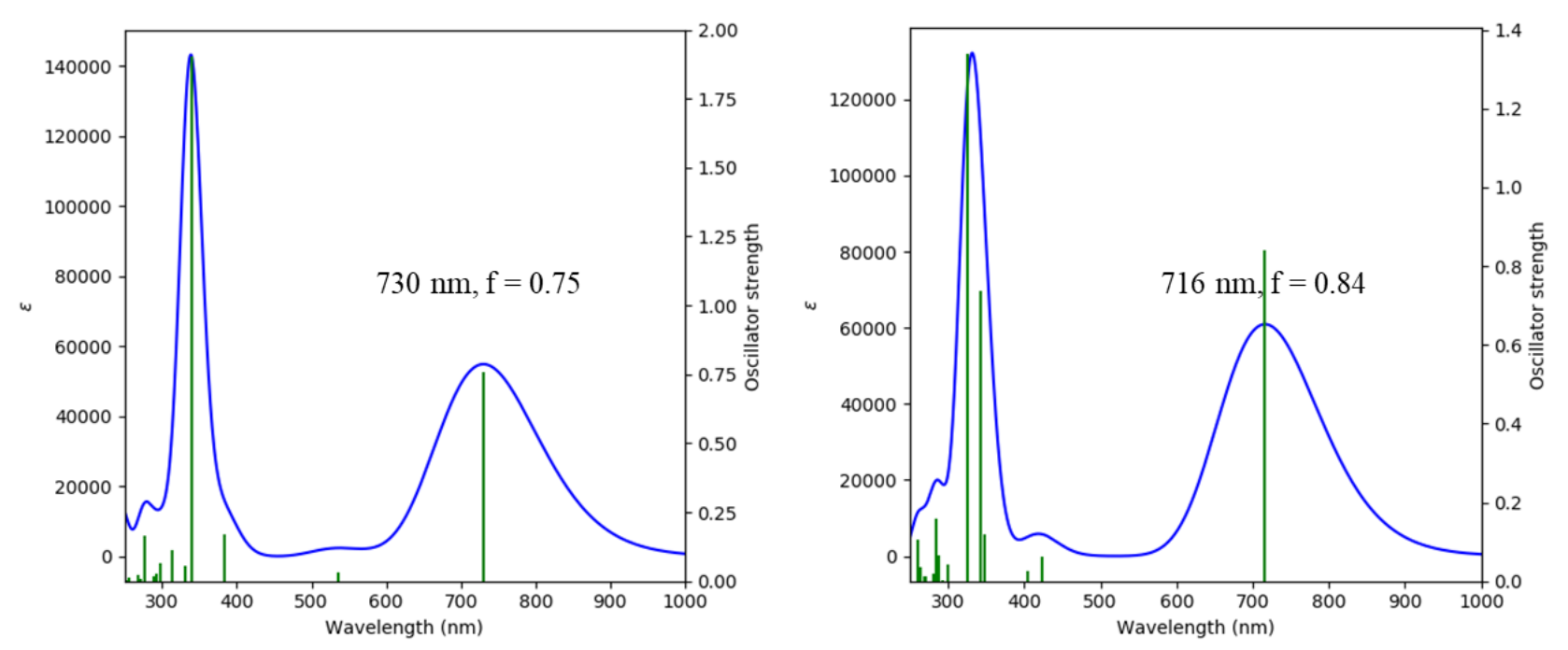

Figure S7. TD-DFT predicted UV-Vis spectra for (left) syn-IIDBT (4) and for (right) synIIDBT-sulfone (8) calculated at the TD-B3LYP/6-311+G* level of theory. 


\section{Additional Electronic Absorption Measurements}

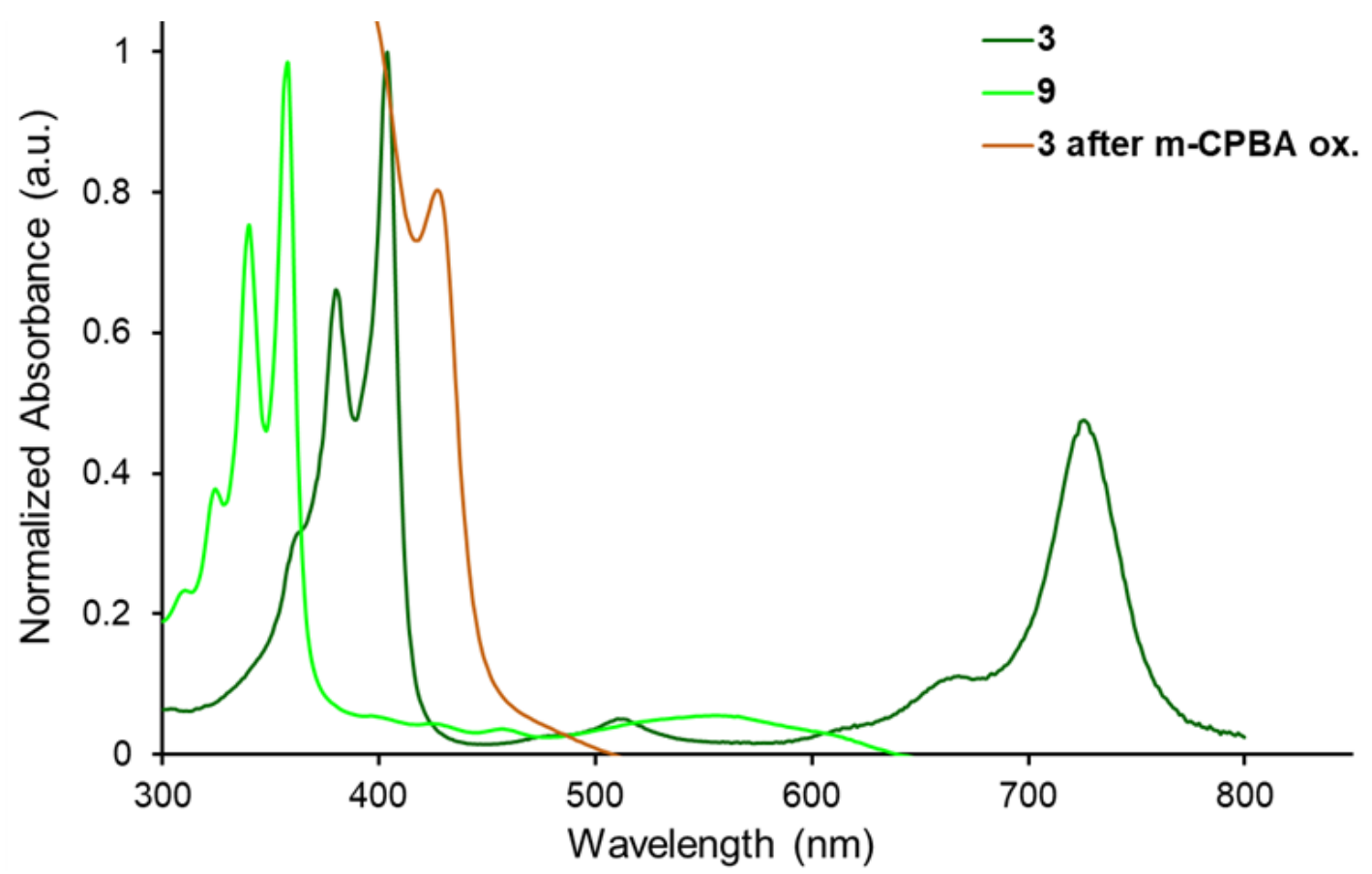

Figure S8. Electronic absorption spectra for anti-IIDBT (3), the sulfonamide side product 9 from the reaction of 3 with UHP, and the orange colored decomposition product that was the result of the unsuccessful oxidation of $\mathbf{3}$ with mCPBA.

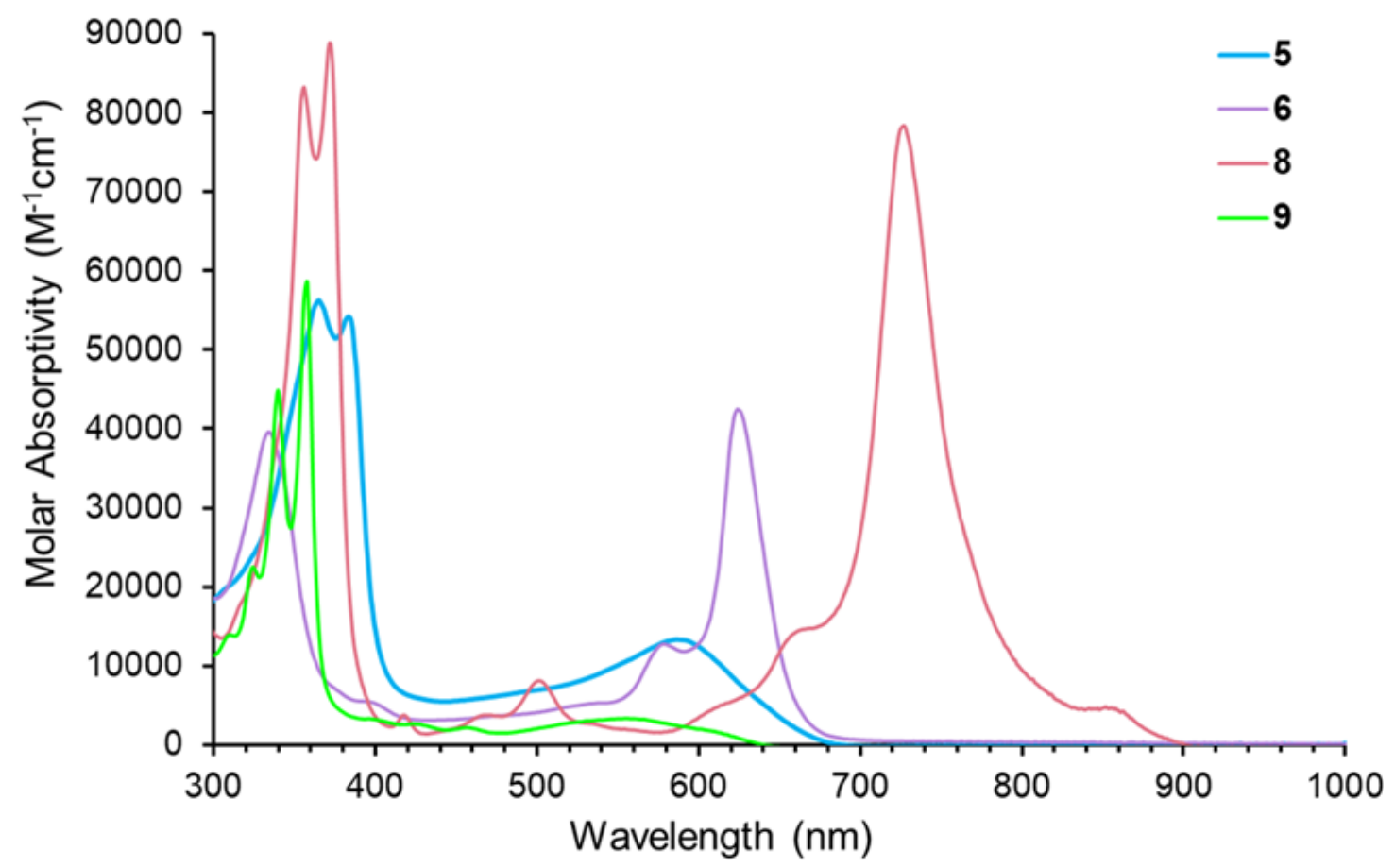

Figure S9. Plot of wavelength vs. molar absorptivity for the three successfully synthesized sulfone compounds $(\mathbf{5}, \mathbf{6}, \mathbf{8})$, and the sulfonamide side product 9 . 


\section{Cyclic Voltammetry}

General. All electrochemical experiments were conducted with traditional 3-electrode geometry using a Biologic SP-50 potentiostat. Electrolyte solutions $(0.1 \mathrm{M})$ were prepared from anhydrous, degassed HPLC grade $\mathrm{CH}_{2} \mathrm{Cl}_{2}$ and anhydrous $\mathrm{Bu}_{4} \mathrm{NPF}_{6}$. The working electrode was a glassy carbon electrode (3-mm diameter), with a Pt-coil counter electrode and a Ag wire pseudo reference. The ferrocene/ferrocenium $(\mathrm{Fc} / \mathrm{Fc}+)$ couple was used as an internal standard following each experiment. Potential values were re-referenced to SCE using a value of 0.46 (V vs. SCE) for the $\mathrm{Fc} / \mathrm{Fc}+$ couple in $\mathrm{CH}_{2} \mathrm{Cl}_{2}$. LUMO and $\mathrm{HOMO}$ levels were approximated using $\mathrm{SCE}=-4.68 \mathrm{eV}$ vs. vacuum. CV experiments were conducted in a three-neck flask that had been evacuated and backfilled with nitrogen for three cycles using standard Schlenk-line technique. Voltammograms were recorded at a sweep rates of $50 \mathrm{mV} \mathrm{s}^{-1}$. E1/2 values were calculated assuming $\mathrm{E}_{1 / 2} \approx$ $E_{o}{ }^{\prime}=\left(E_{\text {anodic }}+E_{\text {cathodic }}\right) / 2$ based on these observations for reversible couples; for irreversible couples the Eo' value is estimated as the potential at peak current. Analyte concentrations were ca. 1-5 mM.
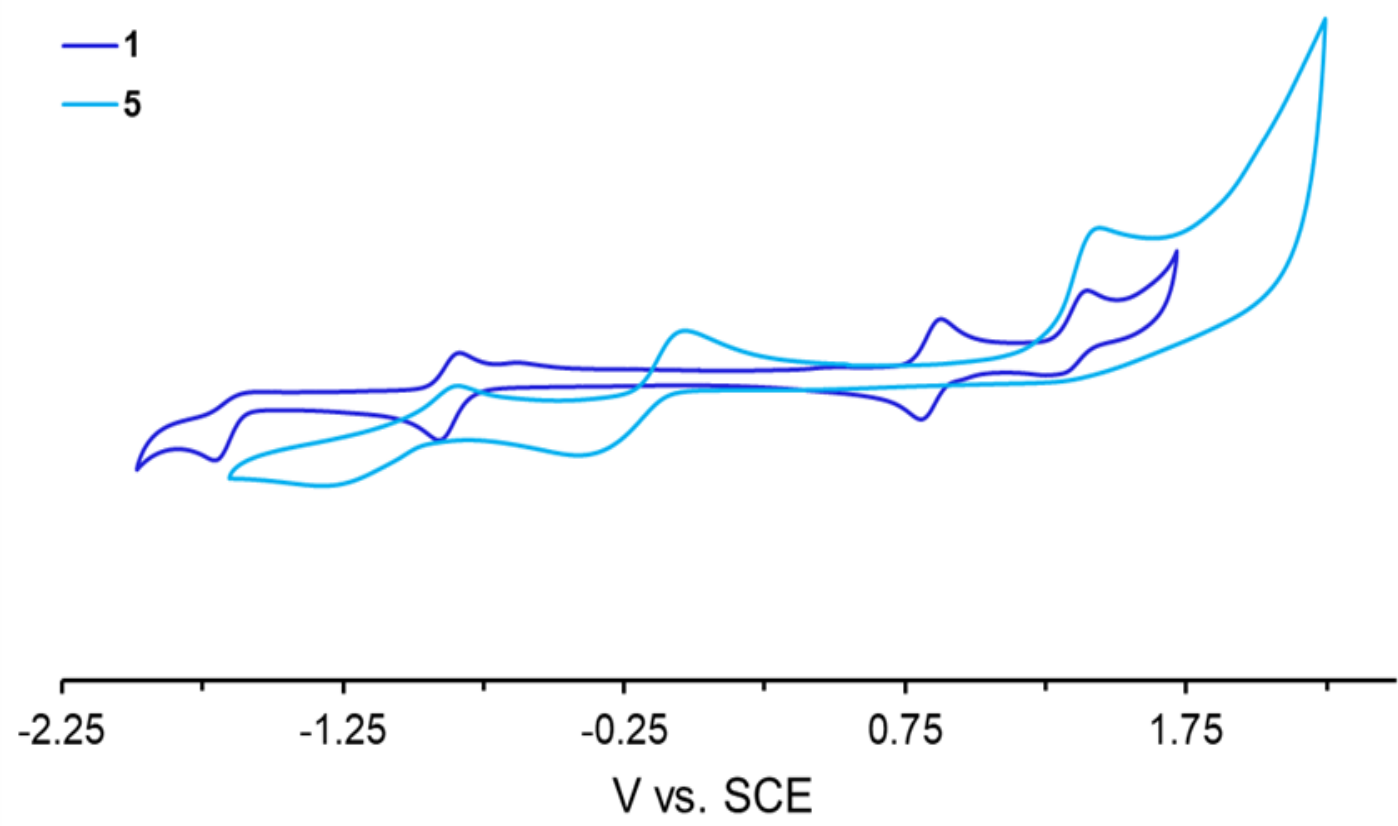

Figure S10.Cyclic voltammograms of 1 and 5. 


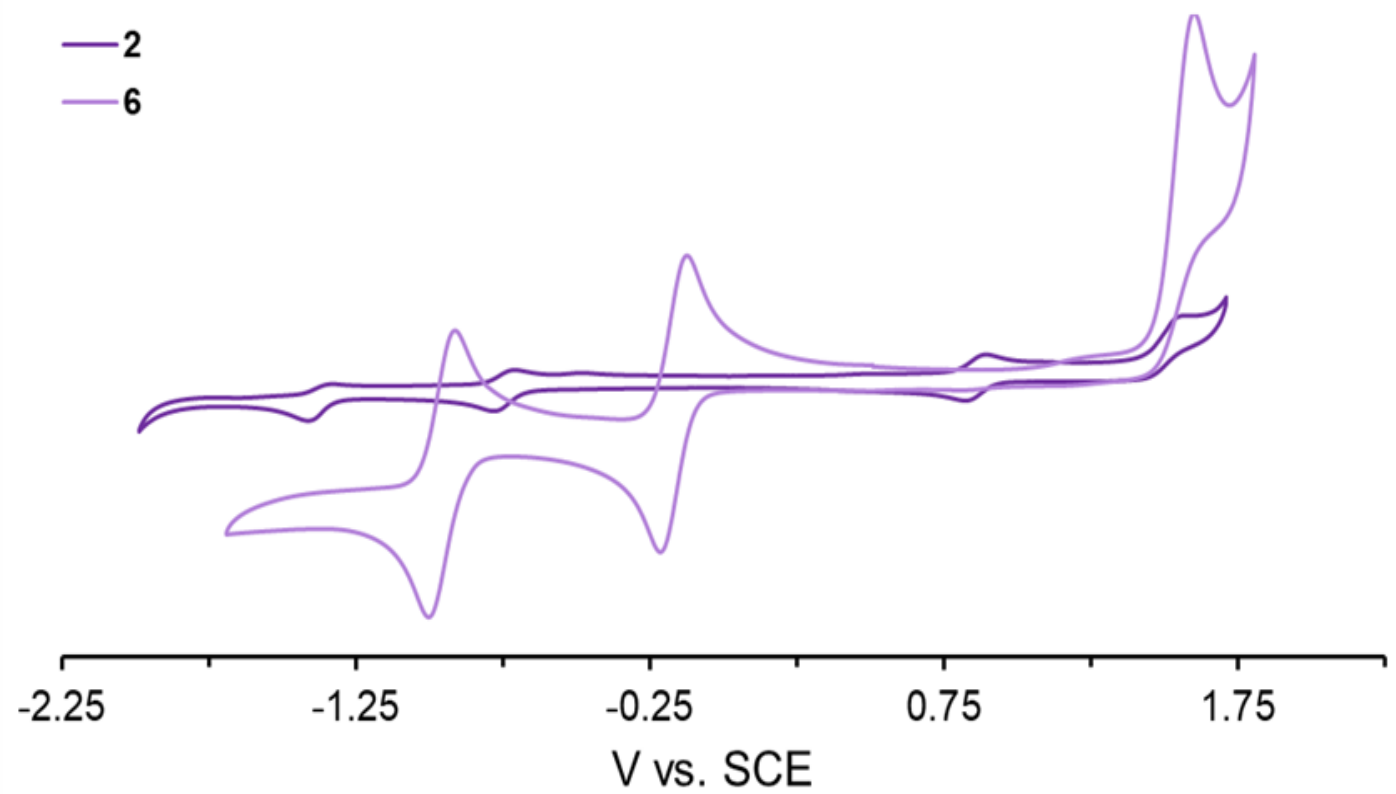

Figure S11.Cyclic voltammograms of 2 and $\mathbf{6 .}$

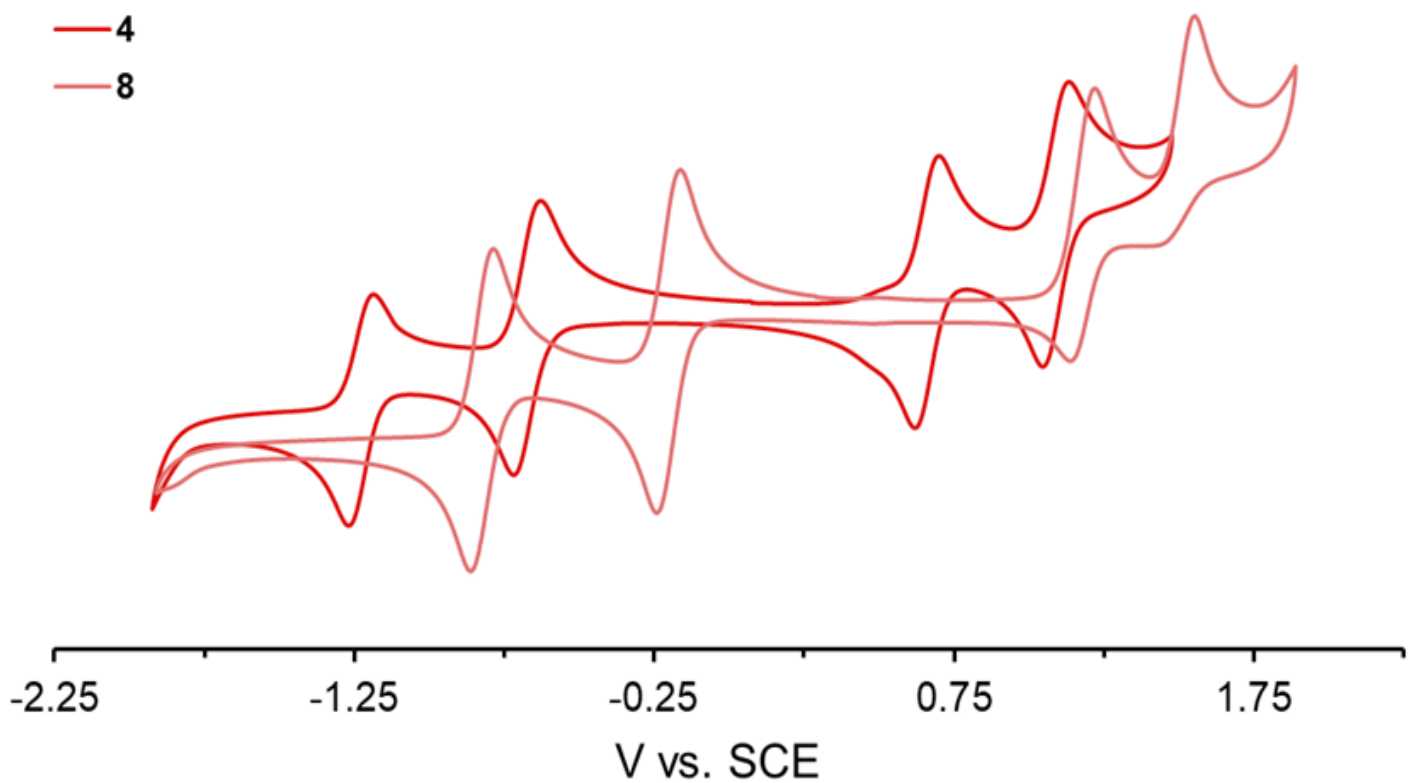

Figure S12.Cyclic voltammograms of $\mathbf{4}$ and 8. 


\section{X-ray Diffraction}

General. Diffraction intensities were collected at $173 \mathrm{~K}$ on a Bruker Apex2 CCD diffractometer using $\operatorname{CuK} \alpha(\lambda=1.54178 \AA$, 5-Mes and 9) or $\operatorname{MoK} \alpha(\lambda=0.71073 \AA, 6)$ radiation. Space groups were determined based on systematic absences (9) and intensity statistics (5-Mes and 6). Absorption corrections were applied by SADABS. ${ }^{1}$ Structures were solved by direct methods and Fourier techniques and refined on $F^{2}$ using full matrix least-squares procedures. All non-H atoms were refined with anisotropic thermal parameters. $\mathrm{H}$ atoms in all structures were refined in calculated positions in a rigid group model. One of two solvent molecules $\mathrm{CHCl}_{3}$ in 9 and a mixture of solvent molecules $\mathrm{CH}_{2} \mathrm{Cl}_{2} / \mathrm{CHCl}_{3} / \mathrm{CH}_{3} \mathrm{CN}$ in $\mathbf{6}$ are highly disordered in a general position and around an inversion center, respectively. These disordered solvent molecules were treated by SQUEEZE. ${ }^{2}$ The corrections of the X-ray data by SQUEEZE are 232 and 208 electron/cell; the required values are 232 electron/cell for four $\mathrm{CHCl}_{3}$ in 9 . The disordered solvent molecules in $\mathbf{6}$ were not resolved and they have not been included into the final formula of the compound given in the CIF file. Thermal parameters for terminal $t$-Bu groups in $\mathbf{6}$ and 9 are significantly elongated. The crystal structure of 9 was treated as a racemic twin; the Flack parameter is $0.28(3)$. X-ray diffraction for crystals of 9 at high angles is very weak due to strong disorder in the structure. As a result the structure of 9 is not very precisely determined. All calculations were performed by the Bruker SHELXL-2014 package. $^{3}$

Low-temperature X-ray diffraction data for $\mathbf{8}$ were collected on a Rigaku XtaLAB Synergy diffractometer coupled to a Rigaku Hypix detector with $\mathrm{Cu} K \alpha$ radiation $(\lambda=1.54184 \AA$ ) from a PhotonJet micro-focus X-ray source at $100 \mathrm{~K}$. The diffraction images were processed and scaled using the CrysAlisPro software. ${ }^{4}$ The structures were solved through intrinsic phasing using SHELXT $^{3}$ and refined against $\mathrm{F}^{2}$ on all data by full-matrix least squares with SHELXL ${ }^{5}$ following established refinement strategies. ${ }^{6}$ All non-hydrogen atoms were refined anisotropically. All hydrogen atoms bound to carbon were included in the model at geometrically calculated positions and refined using a riding model. The isotropic displacement parameters of all hydrogen atoms were fixed to 1.2 times the $\mathrm{U}_{\mathrm{eq}}$ value of the atoms they are linked to (1.5 times for methyl groups).

Crystallographic Data for 5-Mes: $\mathrm{C}_{43} \mathrm{H}_{33} \mathrm{Cl}_{3} \mathrm{O}_{4} \mathrm{~S}_{2}, \mathrm{M}=784.16,0.06 \times 0.04 \times 0.02 \mathrm{~mm}, \mathrm{~T}=$ 173(2) K, Triclinic, space group $P-1, a=8.1459(3) \AA, b=9.0608(4) \AA, c=13.562(5) \AA, \alpha=$ $75.860(2)^{\circ}, \beta=73.582(2)^{\circ}, \gamma=84.176(2)^{\circ}, V=930.52(6) \AA^{3}, Z=1, Z^{\prime}=0.5, D_{c}=1.399 \mathrm{Mg} / \mathrm{m}^{3}$, $\mu(\mathrm{Cu})=3.628 \mathrm{~mm}^{-1}, F(000)=406,2 \theta_{\max }=133.05^{\circ}, 8785$ reflections, 3229 independent reflections 
$\left[\mathrm{R}_{\text {int }}=0.0463\right], \mathrm{R} 1=0.0530, \mathrm{wR} 2=0.1387$ and $\mathrm{GOF}=1.077$ for 3229 reflections ( 255 parameters $)$ with $\mathrm{I}>2 \sigma(\mathrm{I}), \mathrm{R} 1=0.0566, \mathrm{wR} 2=0.1411$ and $\mathrm{GOF}=1.077$ for all reflections, $\max / \mathrm{min}$ residual electron density $+0.662 /-0.451 \mathrm{e}^{-3}$. CCDC 1995028.

Crystallographic Data for 6: $\mathrm{C}_{48} \mathrm{H}_{44} \mathrm{O}_{4} \mathrm{~S}_{2}, \mathrm{M}=748.95,0.06 \times 0.03 \times 0.01 \mathrm{~mm}, \mathrm{~T}=173(2) \mathrm{K}$, Triclinic, space group $P-1, a=13.0977(14) \AA, b=15.0775(16) \AA, c=15.4634(15) \AA, \alpha=$ 115.901(3) $)^{\circ}, \beta=94.740(3)^{\circ}, \gamma=112.307(3)^{\circ}, V=2425.5(4) \AA^{3}, Z=2, Z^{\prime}=1, D_{\mathrm{c}}=1.025 \mathrm{Mg} / \mathrm{m}^{3}$, $\mu(\mathrm{Mo})=0.146 \mathrm{~mm}^{-1}, F(000)=792,2 \theta_{\max }=56.32^{\circ}, 17634$ reflections, 8491 independent reflections $\left[\mathrm{R}_{\text {int }}=0.0485\right], \mathrm{R} 1=0.0848, \mathrm{wR} 2=0.2035$ and $\mathrm{GOF}=1.002$ for 8491 reflections $(487$ parameters) with $\mathrm{I}>2 \sigma(\mathrm{I}), \mathrm{R} 1=0.1468, \mathrm{wR} 2=0.2237$ and $\mathrm{GOF}=1.002$ for all reflections, $\max /$ min residual electron density $+0.465 /-0.389 \mathrm{e}^{-3}$. CCDC 1995030.

Crystallographic Data for 8: $\mathrm{C}_{56} \mathrm{H}_{52} \mathrm{~N}_{2} \mathrm{O}_{4} \mathrm{~S}_{2}, \mathrm{C}_{52} \mathrm{H}_{46} \mathrm{O}_{4} \mathrm{~S}_{2} \cdot 2\left(\mathrm{CH}_{3} \mathrm{CN}\right), \mathrm{M}=881.11,0.136 \mathrm{x}$ $0.058 \times 0.047 \mathrm{~mm}^{3}, \mathrm{~T}=100.00(2) \mathrm{K}$, Triclinic, space group P-1, $a=7.62440(10) \AA, b=10.3907(2)$ $\AA, c=15.0797(3) \AA, \alpha=92.525(2)^{\circ}, \beta=97.786(2)^{\circ}, \gamma=98.462(2)^{\circ}, V=1168.26(4) \AA^{3}, Z=1, D_{c}$ $=1.252 \mathrm{Mg} / \mathrm{m}^{3}, \mu(\mathrm{Cu})=1.418 \mathrm{~mm}^{-1}, F(000)=466,2 \theta_{\max }=74.503^{\circ}, 24859$ reflections, 4765 independent reflections $[\mathrm{R}(\mathrm{int})=0.0318], \mathrm{R} 1=0.0470, \mathrm{wR} 2=0.1063$ and $\mathrm{GOF}=1.020$ for all reflections, max/min residual electron density +0.397/-0.464 e $\AA^{-3}$. CCDC 1994473.

Crystallographic Data for 9: $\mathrm{C}_{54} \mathrm{H}_{48} \mathrm{Cl}_{6} \mathrm{~N}_{2} \mathrm{O}_{4} \mathrm{~S}_{2}, \mathrm{M}=1065.76,0.14 \times 0.06 \times 0.02 \mathrm{~mm}, \mathrm{~T}=$ 173(2) K, Monoclinic, space group $C c, a=11.949(3) \AA, b=25.877(6) \AA, c=16.528(5) \AA, \beta=$ $98.21(2)^{\circ}, V=5058(2) \AA^{3}, Z=4, Z^{\prime}=0.5, D_{\mathrm{c}}=1.400 \mathrm{Mg} / \mathrm{m}^{3}, \mu(\mathrm{Cu})=4.257 \mathrm{~mm}^{-1}, F(000)=2208$, $2 \theta_{\max }=135.86^{\circ}, 17136$ reflections, 6906 independent reflections $\left[\mathrm{R}_{\text {int }}=0.0827\right], \mathrm{R} 1=0.0719$, $\mathrm{wR} 2=0.1746$ and $\mathrm{GOF}=1.052$ for 6906 reflections ( 578 parameters) with $\mathrm{I}>2 \sigma(\mathrm{I}), \mathrm{R} 1=0.0972$, $\mathrm{wR} 2=0.2030$ and $\mathrm{GOF}=1.052$ for all reflections, Flack $=0.28(3), \mathrm{max} / \mathrm{min}$ residual electron density $+0.277 /-0.348 \mathrm{e}^{-3}$. CCDC 1995029 . 


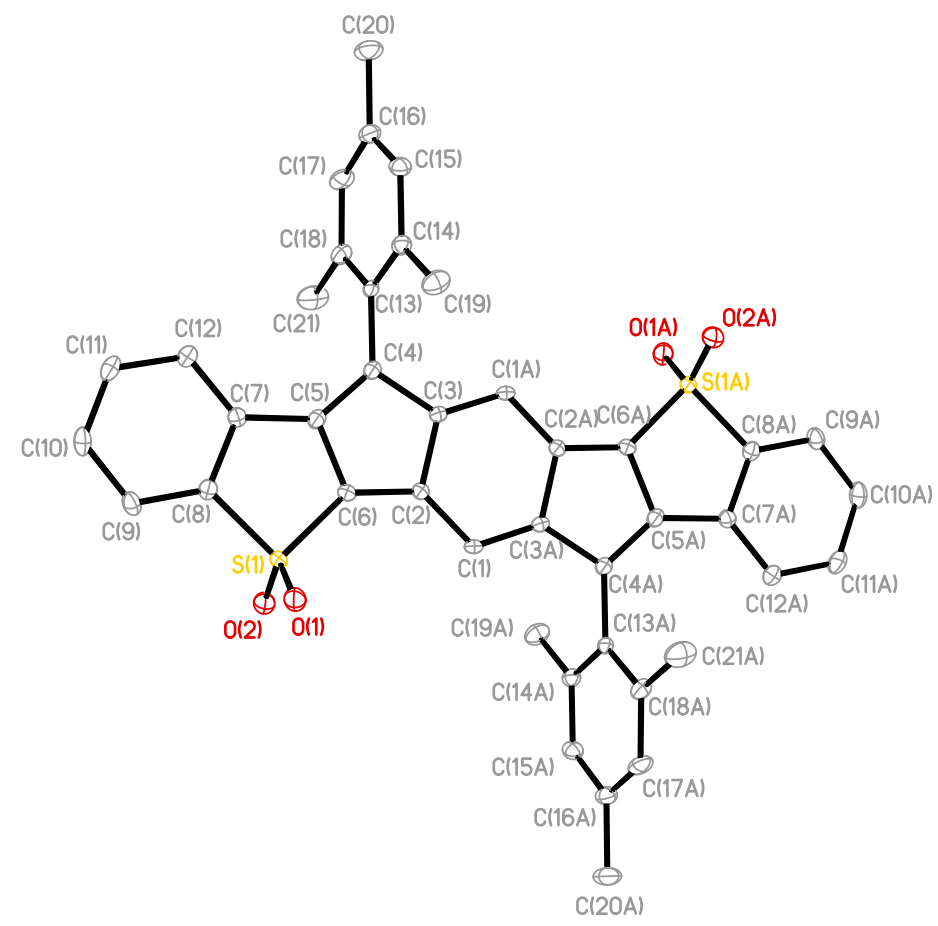

Figure S13. Molecular structure of IDBT-S 5-Mes.

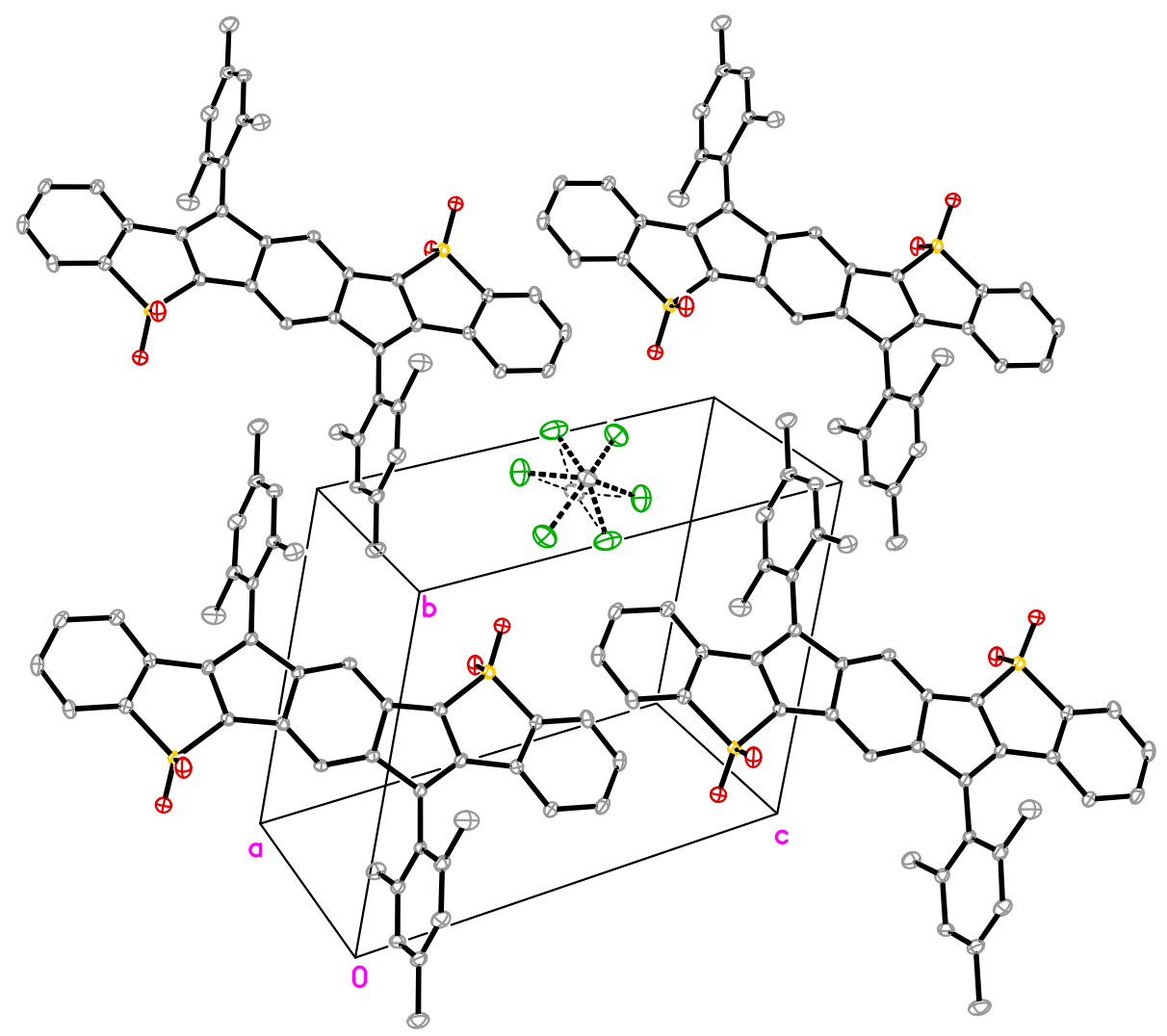

Figure S14. Molecular packing of IDBT-S 5-Mes with disordered solvent molecule. 


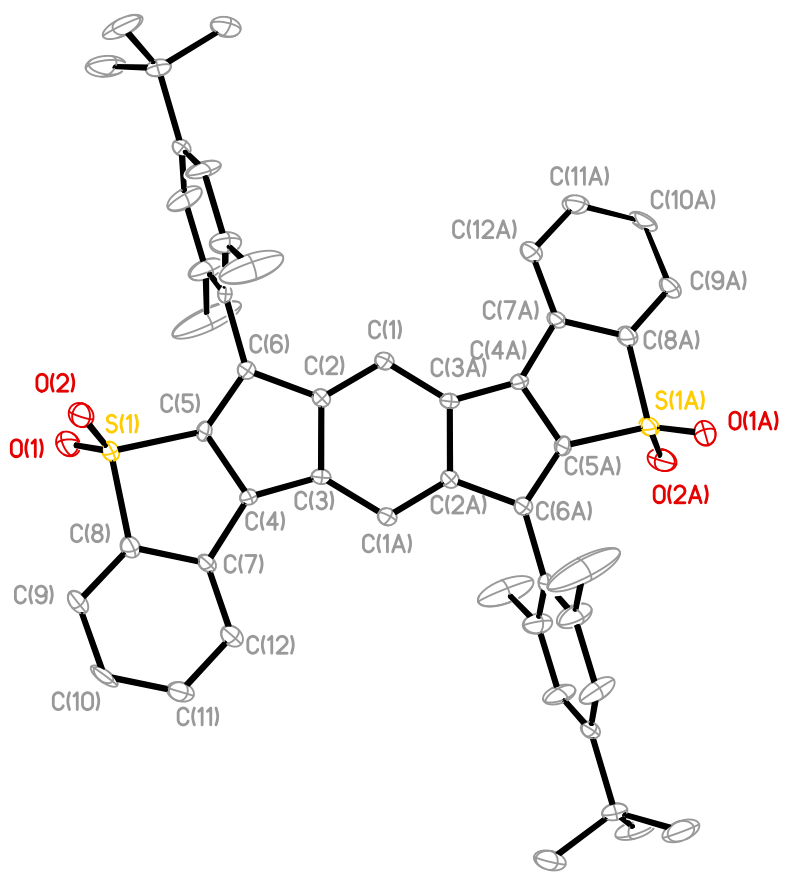

Figure S15. Molecular structure of IDBT-S 6.

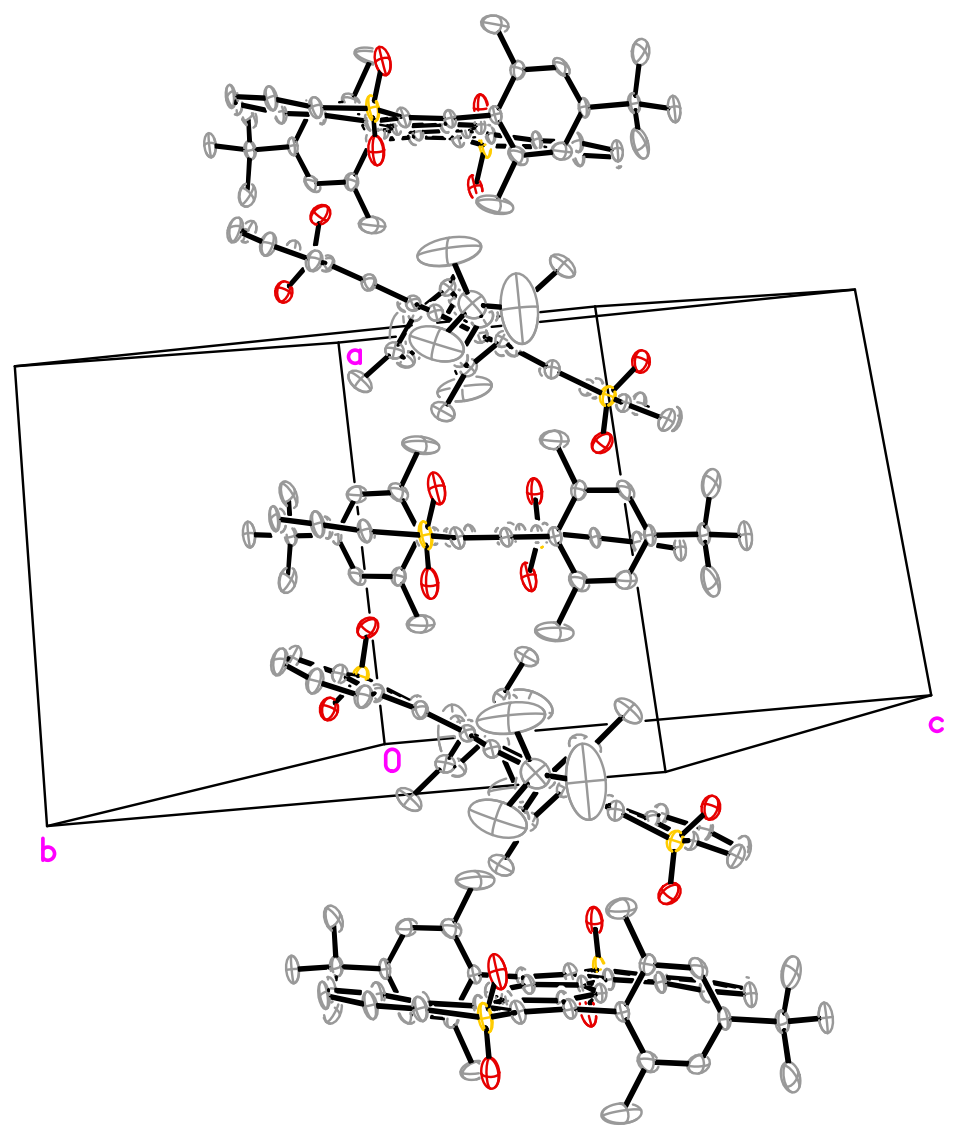

Figure S16. Molecular packing of IDBT-S 6. 


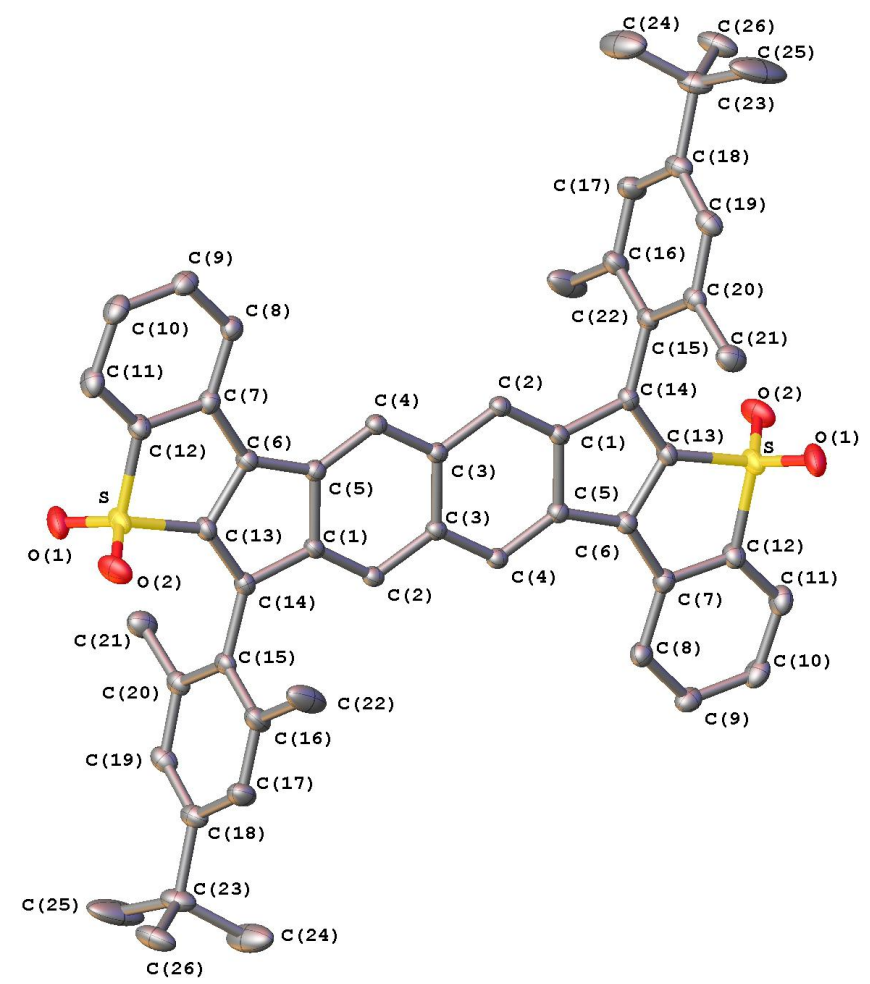

Figure S17. Molecular structure of IDBT-S 8.
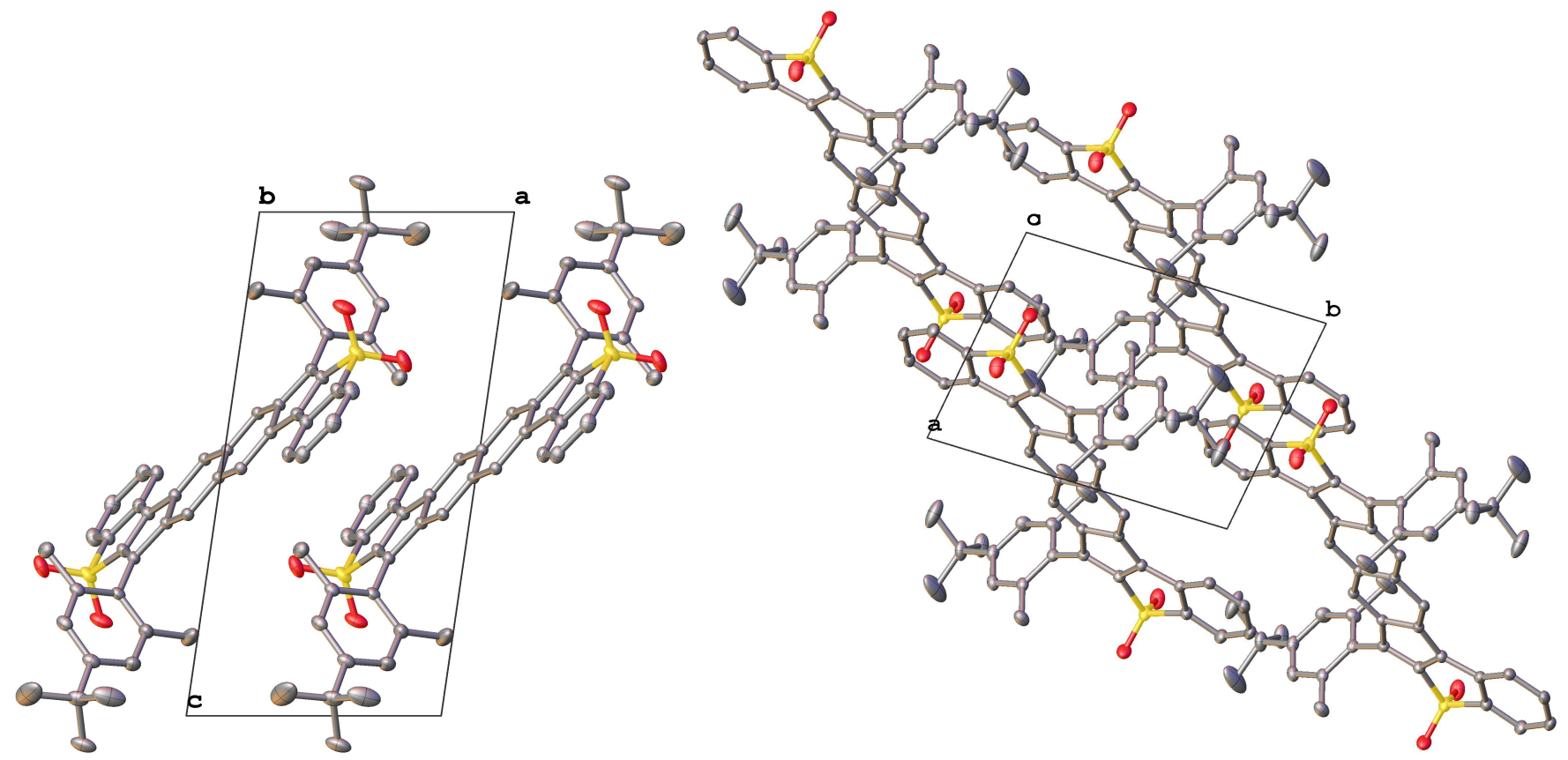

Figure S18. Molecular packing of IDBT-S 8. 


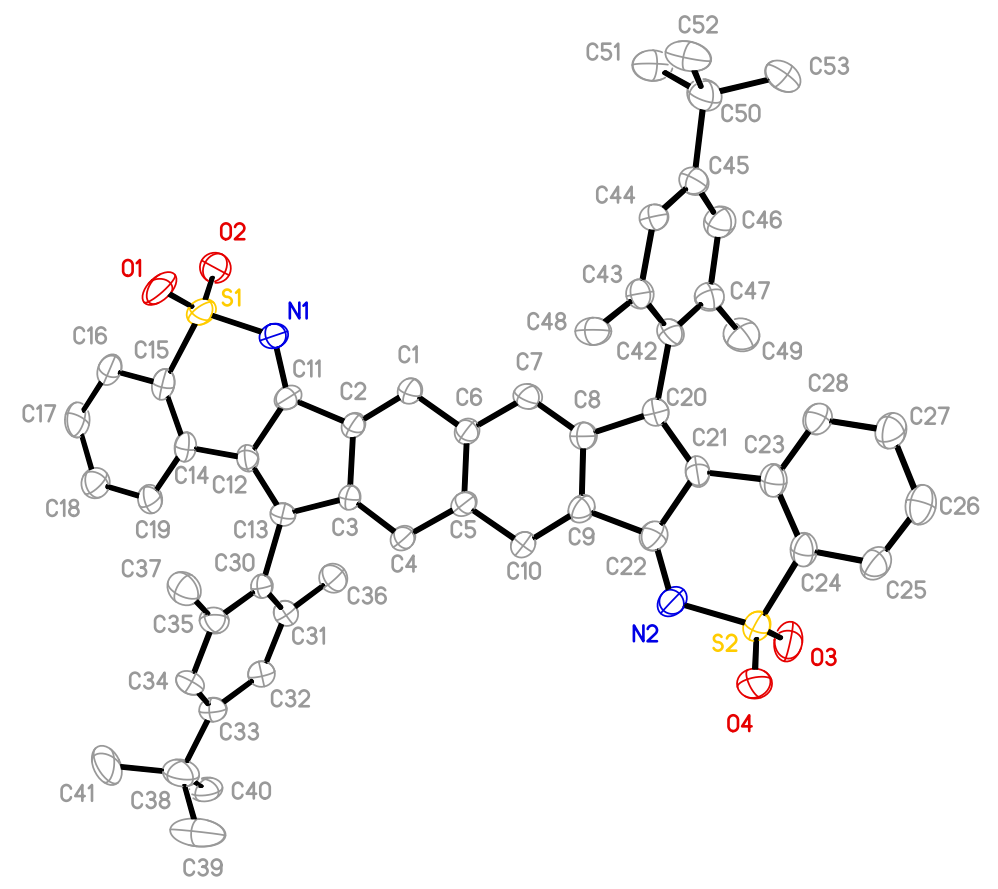

Figure S19. Molecular structure of decomposition product 9.

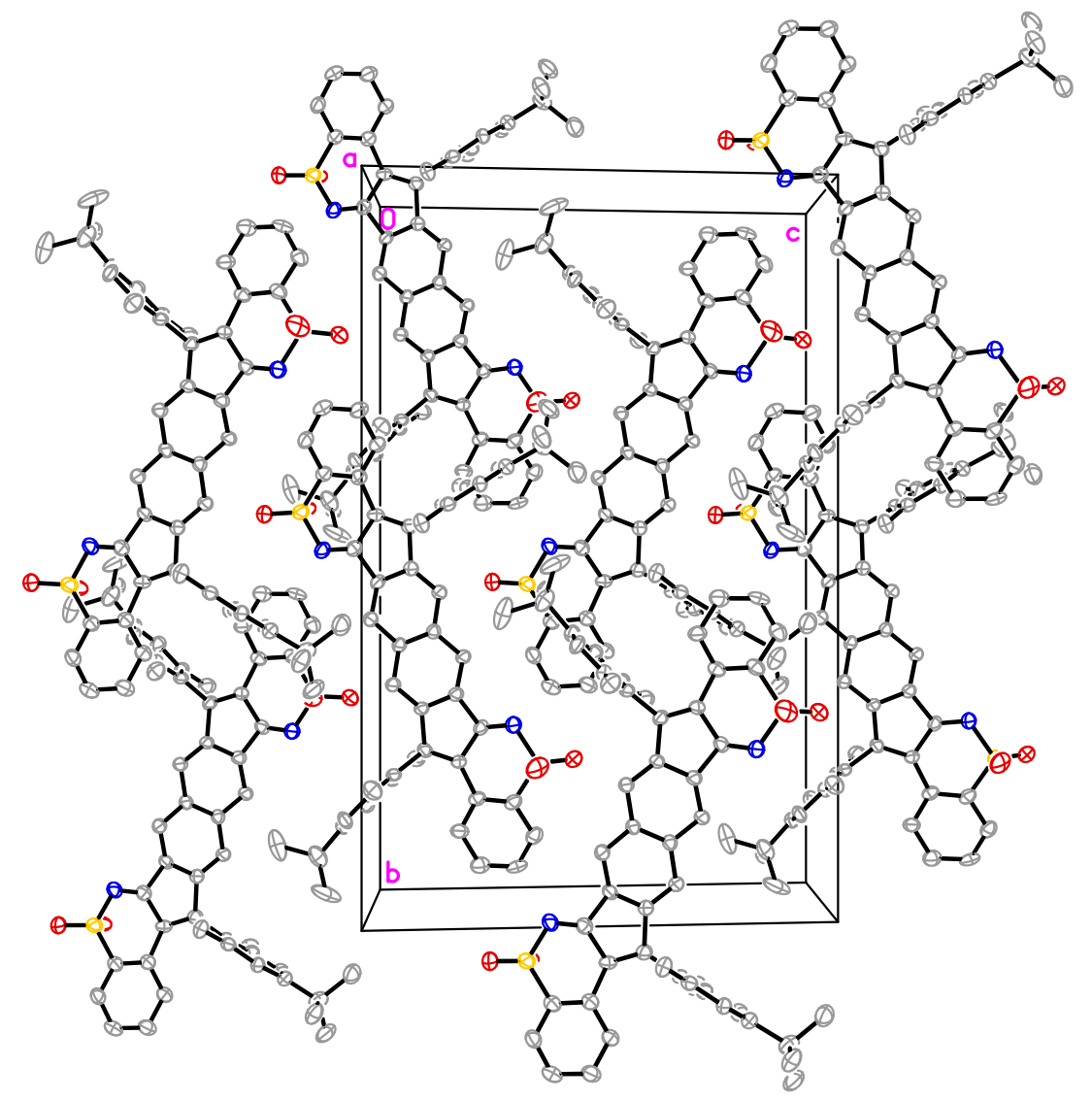

Figure S20. Molecular packing of decomposition product 9. 


\section{Computational Details}

\subsection{NICS-XY scan details}

All calculations related to the NICS-XY scans were performed using Gaussian 09. ${ }^{7}$ Geometries were optimized using B3LYP/6-311++ $\mathrm{G}^{* *}$ level of theory and the structures were verified to be minima by frequency calculation. NICS-XY scans were carried out with the Aroma package $^{8}$ at the B3LYP/6-311++G** level of theory using the sigma-only model. ${ }^{9}$ For the sake of computational efficiency calculations were performed on the hydrocarbon parent molecules for each compound. Previous computational studies in our group have shown that due to their nearly orthogonal orientation relative to the plane of the indacene core, removal of the pendant aryl groups on the apical carbons has little to no effect on NICS-XY scan calculations.
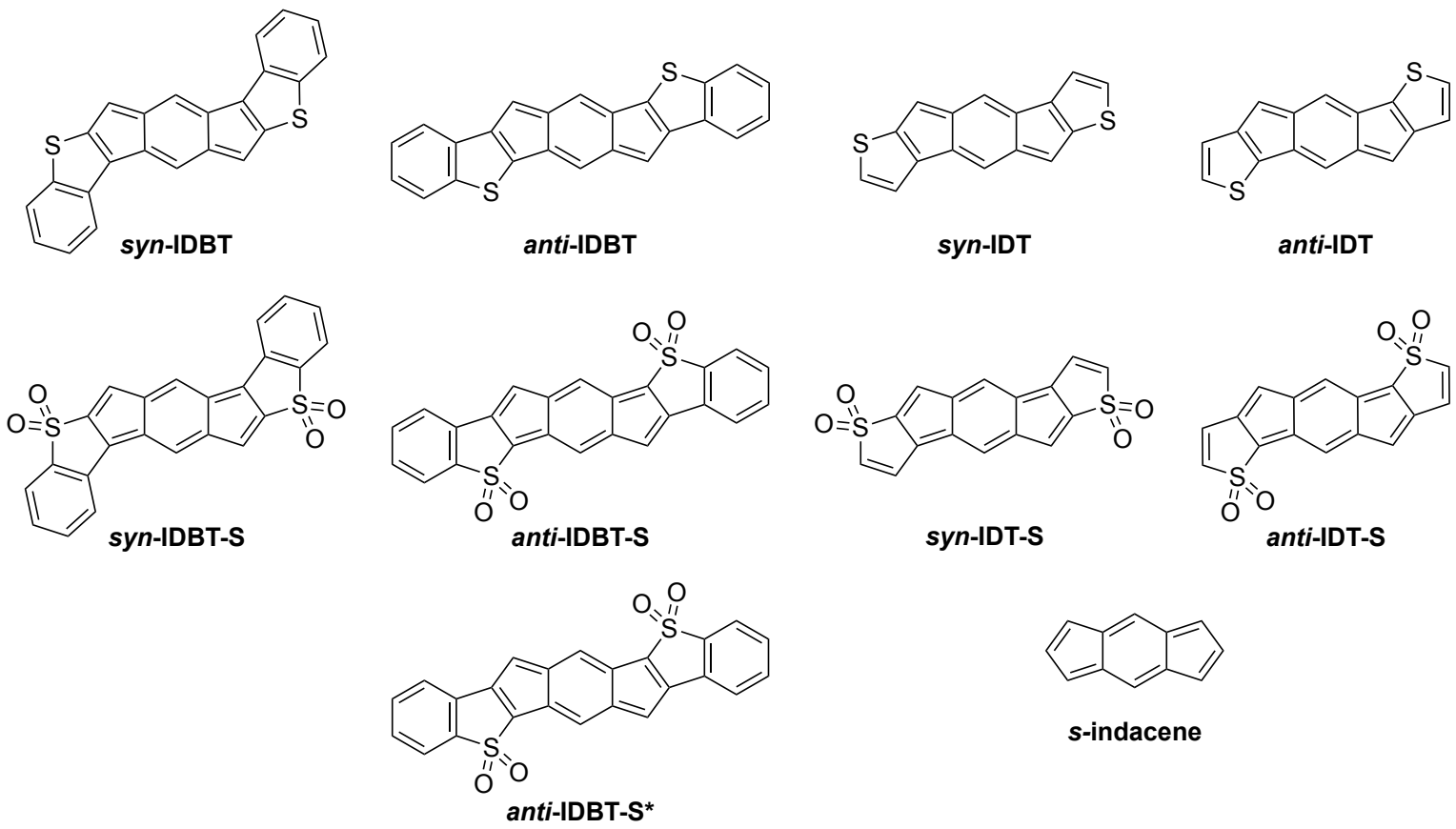

s-indacene

Plunkett's Pentaleno[1,2-a:4,5-a']diacenaphthylene (PDA)
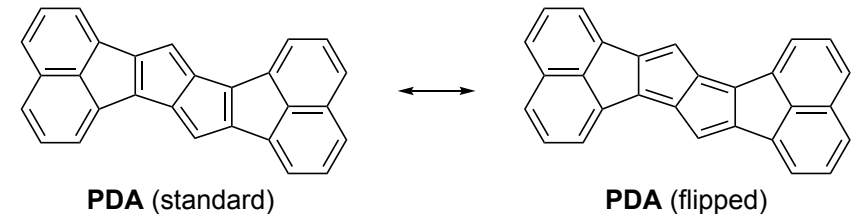

Figure S21. Simplified structures used for the NICS-XY scan calculations. 


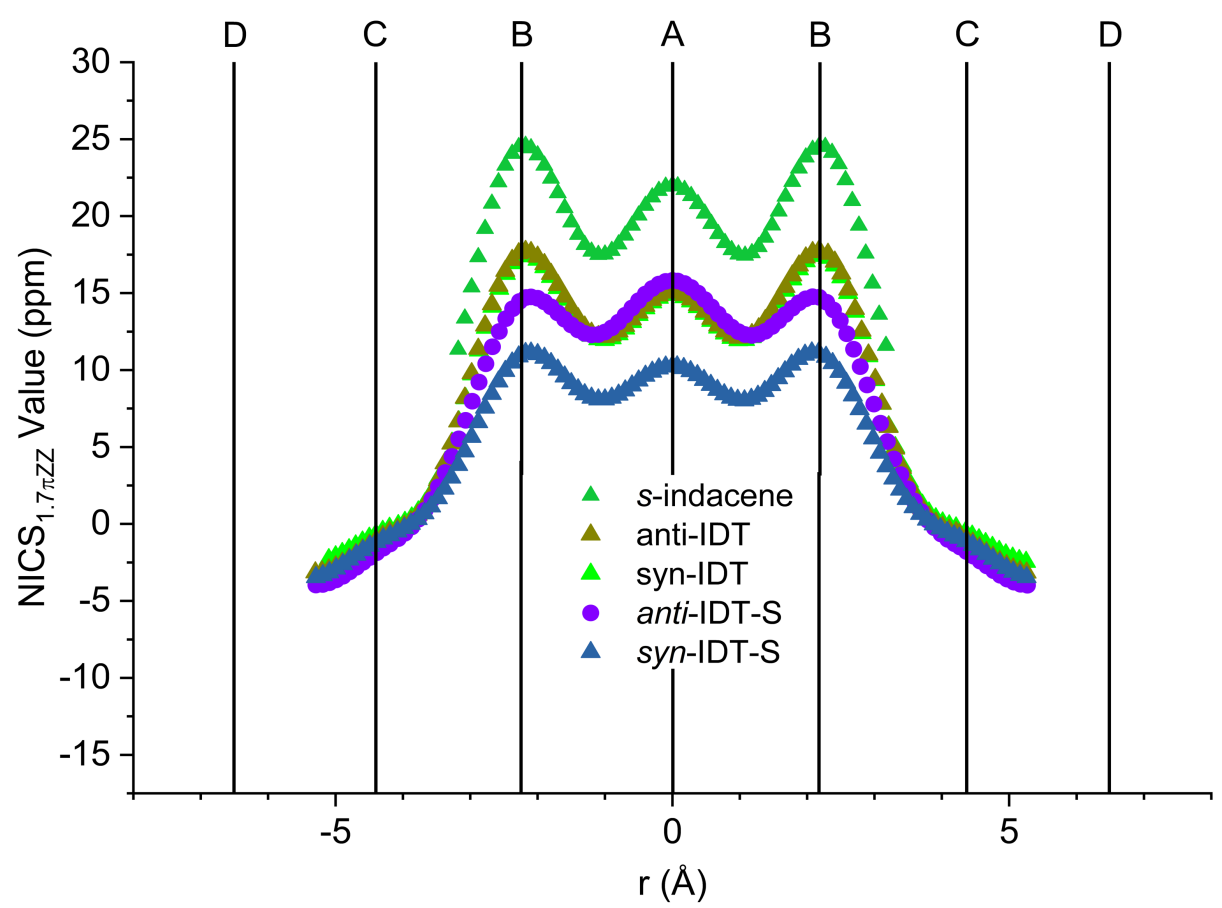

Figure S22. NICS-XY scans of IDTs and IDT-sulfones (IDT-S) from most to least paratropic.

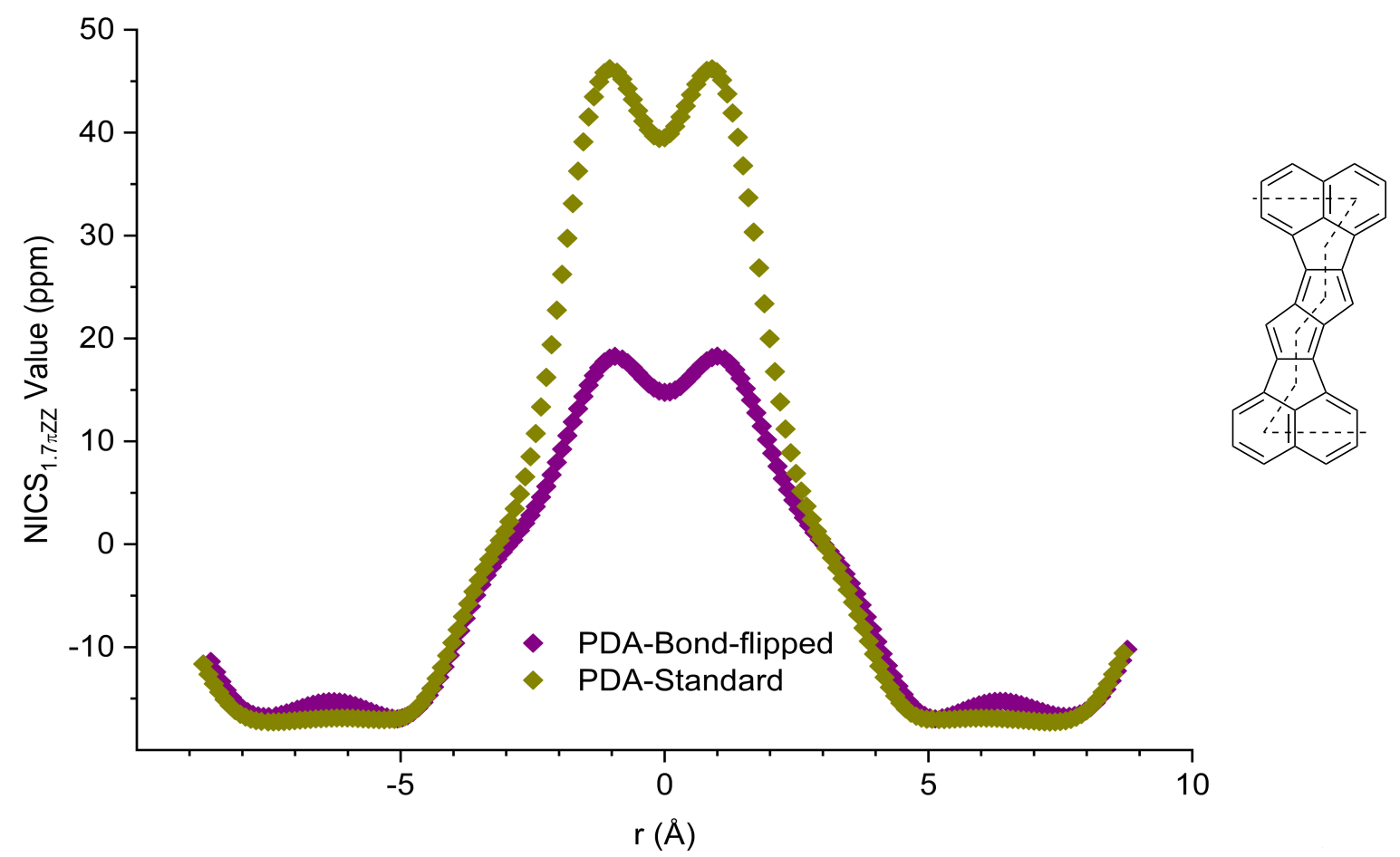

Figure S23. NICS-XY scans (left) of PDA with standard bond alternation vs. PDA with flipped bond alternation along with a depiction of the scan pathway (right). Like the sulfones, the observed bonding pattern is due to the minimization of paratropicity in the pentalene core of the PDAs. It is worth noting that the "standard" geometry is $10.8 \mathrm{kcal} \mathrm{mol}^{-1}$ higher in energy than the "flipped" geometry. 
Calculated Geometries for NICS-XY Scan Structures

\section{$s$-indacene}

Zero-point correction=

0.155426 (Hartree/Particle)

Thermal correction to Energy=

0.163727

Thermal correction to Enthalpy=

0.164671

Thermal correction to Gibbs Free Energy=

Sum of electronic and zero-point Energies=

0.122995

$-461.984810$

$\begin{array}{rrrr}\mathrm{H} & -2.5102810000 & 0.3004220000 & 0.0000000000 \\ \mathrm{H} & -2.4745840000 & -2.6117090000 & 0.0000000000 \\ \mathrm{H} & -1.8282260000 & 3.1575890000 & 0.0000000000 \\ \mathrm{C} & -1.4300430000 & 0.1833990000 & 0.0000000000 \\ \mathrm{C} & -1.4152820000 & -2.3879420000 & 0.0000000000 \\ \mathrm{C} & -0.8486790000 & -1.1094930000 & 0.0000000000 \\ \mathrm{C} & -0.8486790000 & 2.6984970000 & 0.0000000000 \\ \mathrm{C} & -0.5912530000 & 1.2773850000 & 0.0000000000 \\ \mathrm{H} & -0.5068920000 & -4.4309980000 & 0.0000000000 \\ \mathrm{C} & -0.3648030000 & -3.3598230000 & 0.0000000000 \\ \mathrm{C} & 0.3648030000 & 3.3598230000 & 0.0000000000 \\ \mathrm{H} & 0.5068920000 & 4.4309980000 & 0.0000000000 \\ \mathrm{C} & 0.5912530000 & -1.2773850000 & 0.0000000000 \\ \mathrm{C} & 0.8486790000 & 1.1094930000 & 0.0000000000 \\ \mathrm{C} & 0.8486790000 & -2.6984970000 & 0.0000000000 \\ \mathrm{C} & 1.4152820000 & 2.3879420000 & 0.0000000000 \\ \mathrm{C} & 1.4300430000 & -0.1833990000 & 0.0000000000 \\ \mathrm{H} & 1.8282260000 & -3.1575890000 & 0.0000000000 \\ \mathrm{H} & 2.4745840000 & 2.6117090000 & 0.0000000000 \\ \mathrm{H} & 2.5102810000 & -0.3004220000 & 0.0000000000\end{array}$

\section{anti-IDBT (1)}

Zero-point correction=

Thermal correction to Energy=

0.276505 (Hartree/Particle)

0.295053

Thermal correction to Enthalpy=

0.295997

Thermal correction to Gibbs Free Energy $=\quad 0.230158$

Sum of electronic and zero-point Energies $=\quad-1718.131387$

$\begin{array}{llll}\text { H } & -6.1286300000 & -3.8515800000 & 0.0000000000 \\ \text { H } & -5.8051950000 & -6.3091470000 & 0.0000000000 \\ \text { C } & -5.1300770000 & -4.2730740000 & 0.0000000000 \\ \text { C } & -4.9428860000 & -5.6527350000 & 0.0000000000 \\ \text { S } & -4.0178190000 & -1.6725420000 & 0.0000000000 \\ \text { C } & -4.0062750000 & -3.4519740000 & 0.0000000000\end{array}$




$\begin{array}{lrrr}\mathrm{C} & -3.6510580000 & -6.1963720000 & 0.0000000000 \\ \mathrm{H} & -3.5247130000 & -7.2730020000 & 0.0000000000 \\ \mathrm{C} & -2.6900680000 & -3.9801160000 & 0.0000000000 \\ \mathrm{C} & -2.5316060000 & -5.3738130000 & 0.0000000000 \\ \mathrm{C} & -2.2858450000 & -1.6429150000 & 0.0000000000 \\ \mathrm{H} & -2.1736340000 & 1.2944130000 & 0.0000000000 \\ \mathrm{C} & -1.7111180000 & -2.9239560000 & 0.0000000000 \\ \mathrm{H} & -1.5366610000 & -5.8048880000 & 0.0000000000 \\ \mathrm{C} & -1.2466520000 & 0.7287100000 & 0.0000000000 \\ \mathrm{C} & -1.2466520000 & -0.6490180000 & 0.0000000000 \\ \mathrm{H} & -0.4313600000 & 3.5747470000 & 0.0000000000 \\ \mathrm{C} & -0.2953890000 & -2.7729320000 & 0.0000000000 \\ \mathrm{C} & -0.0044970000 & 1.4034920000 & 0.0000000000 \\ \mathrm{C} & 0.0044970000 & -1.4034920000 & 0.0000000000 \\ \mathrm{C} & 0.2953890000 & 2.7729320000 & 0.0000000000 \\ \mathrm{H} & 0.4313600000 & -3.5747470000 & 0.0000000000 \\ \mathrm{C} & 1.2466520000 & -0.7287100000 & 0.0000000000 \\ \mathrm{C} & 1.2466520000 & 0.6490180000 & 0.0000000000 \\ \mathrm{H} & 1.5366610000 & 5.8048880000 & 0.0000000000 \\ \mathrm{C} & 1.7111180000 & 2.9239560000 & 0.0000000000 \\ \mathrm{H} & 2.1736340000 & -1.2944130000 & 0.0000000000 \\ \mathrm{C} & 2.2858450000 & 1.6429150000 & 0.0000000000 \\ \mathrm{C} & 2.5316060000 & 5.3738130000 & 0.0000000000 \\ \mathrm{C} & 2.6900680000 & 3.9801160000 & 0.0000000000 \\ \mathrm{H} & 3.5247130000 & 7.2730020000 & 0.0000000000 \\ \mathrm{C} & 3.6510580000 & 6.1963720000 & 0.0000000000 \\ \mathrm{C} & 4.0062750000 & 3.4519740000 & 0.0000000000 \\ \mathrm{~S} & 4.0178190000 & 1.6725420000 & 0.0000000000 \\ \mathrm{C} & 4.9428860000 & 5.6527350000 & 0.0000000000 \\ \mathrm{C} & 5.1300770000 & 4.2730740000 & 0.0000000000 \\ \mathrm{H} & 5.8051950000 & 6.3091470000 & 0.0000000000 \\ \mathrm{H} & 6.1286300000 & 3.8515800000 & 0.0000000000\end{array}$

\section{syn-IDBT (2)}

Zero-point correction $=$

Thermal correction to Energy=

Thermal correction to Enthalpy=

Thermal correction to Gibbs Free Energy=

Sum of electronic and zero-point Energies=

0.276857 (Hartree/Particle)

0.295366

0.296310

0.230524

$-1718.129179$

$\begin{array}{llll}\mathrm{H} & -7.5190140000 & -2.6827280000 & 0.00000000000 \\ \mathrm{H} & -6.8201300000 & -0.3069460000 & 0.0000000000 \\ \mathrm{C} & -6.4642940000 & -2.4333600000 & 0.0000000000\end{array}$




$\begin{array}{lrrr}\mathrm{C} & -6.0661100000 & -1.0856680000 & 0.0000000000 \\ \mathrm{H} & -5.8282160000 & -4.4909360000 & 0.0000000000 \\ \mathrm{C} & -5.5193320000 & -3.4520420000 & 0.0000000000 \\ \mathrm{C} & -4.7245740000 & -0.7416500000 & 0.0000000000 \\ \mathrm{H} & -4.4309630000 & 0.3011920000 & 0.0000000000 \\ \mathrm{C} & -4.1676710000 & -3.1098100000 & 0.0000000000 \\ \mathrm{C} & -3.7405750000 & -1.7495270000 & 0.0000000000 \\ \mathrm{~S} & -2.8145630000 & -4.2456770000 & 0.0000000000 \\ \mathrm{C} & -2.3147730000 & -1.6615170000 & 0.0000000000 \\ \mathrm{H} & -2.1777890000 & 1.2834010000 & 0.0000000000 \\ \mathrm{C} & -1.6920510000 & -2.9080290000 & 0.0000000000 \\ \mathrm{C} & -1.2558300000 & 0.7114710000 & 0.0000000000 \\ \mathrm{C} & -1.2558300000 & -0.6576730000 & 0.0000000000 \\ \mathrm{H} & -0.4561280000 & 3.5603190000 & 0.0000000000 \\ \mathrm{C} & -0.2720290000 & -2.7607780000 & 0.0000000000 \\ \mathrm{C} & -0.0051050000 & 1.3954320000 & 0.0000000000 \\ \mathrm{C} & 0.0051050000 & -1.3954320000 & 0.0000000000 \\ \mathrm{C} & 0.2720290000 & 2.7607780000 & 0.0000000000 \\ \mathrm{H} & 0.4561280000 & -3.5603190000 & 0.0000000000 \\ \mathrm{C} & 1.2558300000 & -0.7114710000 & 0.0000000000 \\ \mathrm{C} & 1.2558300000 & 0.6576730000 & 0.0000000000 \\ \mathrm{C} & 1.6920510000 & 2.9080290000 & 0.0000000000 \\ \mathrm{H} & 2.1777890000 & -1.2834010000 & 0.0000000000 \\ \mathrm{C} & 2.3147730000 & 1.6615170000 & 0.0000000000 \\ \mathrm{~S} & 2.8145630000 & 4.2456770000 & 0.0000000000 \\ \mathrm{C} & 3.7405750000 & 1.7495270000 & 0.0000000000 \\ \mathrm{C} & 4.1676710000 & 3.1098100000 & 0.0000000000 \\ \mathrm{H} & 4.4309630000 & -0.3011920000 & 0.0000000000 \\ \mathrm{C} & 4.7245740000 & 0.7416500000 & 0.0000000000 \\ \mathrm{C} & 5.5193320000 & 3.4520420000 & 0.0000000000 \\ \mathrm{H} & 5.8282160000 & 4.4909360000 & 0.0000000000 \\ \mathrm{C} & 6.0661100000 & 1.0856680000 & 0.0000000000 \\ \mathrm{H} & 6.4642940000 & 2.4333600000 & 0.0000000000 \\ & 6.8201300000 & 0.3069460000 & 0.0000000000 \\ & 7.5190140000 & 2.6827280000 & 0.0000000000\end{array}$

anti-IDBT-sulfone (bond 'flipped', 5)

Zero-point correction= 0.293661 (Hartree/Particle)

Thermal correction to Energy=

0.316228

Thermal correction to Enthalpy=

0.317172

Thermal correction to Gibbs Free Energy = $\quad 0.241864$

Sum of electronic and zero-point Energies $=\quad-2018.916229$

H $\quad-4.7226432920 \quad-6.5591048234 \quad 0.0000000000$ 


\begin{tabular}{|c|c|c|c|}
\hline & -4.3881243537 & -4.1092432870 & \\
\hline & -3.7166097002 & -6.1557452115 & 0.0000000000 \\
\hline & -3.5330952354 & -4.77521 & .0000000000 \\
\hline & -3.4074056047 & -1.3545659945 & 0.0000000000 \\
\hline & -2.7901258152 & -8.1009186597 & 0.000000000 \\
\hline & -2.6252790079 & -7.0301 & 0.0000000000 \\
\hline & -2.3573134435 & -1.610 & 0.0000000000 \\
\hline & -2.2356373255 & -4.25 & 0000000 \\
\hline & -2.18 & 1.2 & \\
\hline & -1.8020124868 & -2.86 & 000 \\
\hline & -1.3223 & -6.5 & 000 \\
\hline & -1.2647514722 & 0.7 & 000 \\
\hline & -1.2 & -0.6 & 000 \\
\hline & -1.15 & & \\
\hline ) & -1.156 & 4.5 & \\
\hline & -1.1 & -5.1 & 000 \\
\hline & -0.4 & -7.1 & \\
\hline & -0.44 & 4.3 & 0000 \\
\hline & -0.3 & -2.7 & 000 \\
\hline & -0.0 & -1.3 & \\
\hline & 0.00 & 1.3 & 000 \\
\hline & 682 & 2.7 & 000 \\
\hline & 0.44 & -4.3 & 000 \\
\hline & 6224 & 0286 & 000 \\
\hline & 1.15 & -4.5 & 992 \\
\hline 0 & 79502 & -4.5 & \\
\hline & 1.15 & & \\
\hline & 43701 & 0.6 & 000 \\
\hline & 14722 & -0.7 & 000 \\
\hline & 1.32 & & \\
\hline & 1.8020124868 & 2.8 & 0000000 \\
\hline & 2.18 & -1.2 & 0000 \\
\hline & 2.235 & 4.2 & 00000000 \\
\hline & 2.3573134435 & 1.6 & \\
\hline & 90079 & 7.0 & 0000000 \\
\hline & 2.7901258152 & & 0.0000000000 \\
\hline & 3.4074056047 & 1.3 & \\
\hline & 3.5330952354 & 2135921 & 0.0000000000 \\
\hline & 3.7166097002 & 6.1557452115 & 0.0000000000 \\
\hline & 4.3881243537 & 4.1092432870 & 0.000000000 \\
\hline & 4.7226432920 & 6.5591048234 & 0.00000000 \\
\hline
\end{tabular}


anti-IDBT-sulfone (regular geometry, 5*)

Zero-point correction=

Thermal correction to Energy= Thermal correction to Enthalpy= Thermal correction to Gibbs Free Energy= Sum of electronic and zero-point Energies=
0.293098 (Hartree/Particle)

0.315801

0.316745

0.240492

$-2018.913837$

$\begin{array}{lccc}\mathrm{H} & -8.5866580000 & 0.6443890000 & 0.0000000000 \\ \mathrm{H} & -7.5395030000 & 2.8816740000 & 0.0000000000 \\ \mathrm{C} & -7.5070990000 & 0.7352990000 & 0.0000000000 \\ \mathrm{H} & -7.1628970000 & -1.4056840000 & 0.0000000000 \\ \mathrm{C} & -6.9134730000 & 1.9968970000 & 0.0000000000 \\ \mathrm{C} & -6.7148040000 & -0.4192040000 & 0.0000000000 \\ \mathrm{C} & -5.5233710000 & 2.1411690000 & 0.0000000000 \\ \mathrm{C} & -5.3444540000 & -0.2569470000 & 0.0000000000 \\ \mathrm{H} & -5.0770810000 & 3.1289790000 & 0.0000000000 \\ \mathrm{C} & -4.7209540000 & 1.0018010000 & 0.0000000000 \\ \mathrm{O} & -4.1499210000 & -2.3415690000 & 1.2601950000 \\ \mathrm{O} & -4.1499210000 & -2.3415690000 & -1.2601950000 \\ \mathrm{~S} & -4.1115690000 & -1.5944110000 & 0.0000000000 \\ \mathrm{C} & -3.2621510000 & 0.8839370000 & 0.0000000000 \\ \mathrm{C} & -2.7817060000 & -0.4086570000 & 0.0000000000 \\ \mathrm{H} & -2.1784640000 & 2.8570720000 & 0.0000000000 \\ \mathrm{C} & -2.1357180000 & 1.7760970000 & 0.0000000000 \\ \mathrm{C} & -1.3452760000 & -0.4036100000 & 0.0000000000 \\ \mathrm{C} & -0.9730070000 & 1.0068630000 & 0.0000000000 \\ \mathrm{H} & -0.6711700000 & -2.4451340000 & 0.0000000000 \\ \mathrm{C} & -0.3957150000 & -1.3957970000 & 0.0000000000 \\ \mathrm{C} & 0.3957150000 & 1.3957970000 & 0.0000000000 \\ \mathrm{H} & 0.6711700000 & 2.4451340000 & 0.0000000000 \\ \mathrm{C} & 0.9730070000 & -1.0068630000 & 0.0000000000 \\ \mathrm{C} & 1.3452760000 & 0.4036100000 & 0.0000000000 \\ \mathrm{C} & 2.1357180000 & -1.7760970000 & 0.0000000000 \\ \mathrm{H} & 2.1784640000 & -2.8570720000 & 0.0000000000 \\ \mathrm{C} & 2.7817060000 & 0.4086570000 & 0.0000000000 \\ \mathrm{C} & 3.2621510000 & -0.8839370000 & 0.0000000000 \\ \mathrm{~S} & 4.1115690000 & 1.5944110000 & 0.0000000000 \\ \mathrm{O} & 4.1499210000 & 2.3415690000 & 1.2601950000 \\ \mathrm{O} & 4.1499210000 & 2.3415690000 & -1.2601950000 \\ \mathrm{C} & 4.7209540000 & -1.0018010000 & 0.0000000000 \\ \mathrm{H} & 5.0770810000 & -3.1289790000 & 0.0000000000 \\ \mathrm{C} & 5.3444540000 & 0.2569470000 & 0.0000000000 \\ \mathrm{C} & 5.5233710000 & -2.1411690000 & 0.0000000000 \\ \mathrm{C} & 6.7148040000 & 0.4192040000 & 0.0000000000\end{array}$




$\begin{array}{lrrr}\text { C } & 6.9134730000 & -1.9968970000 & 0.0000000000 \\ \text { H } & 7.1628970000 & 1.4056840000 & 0.0000000000 \\ \text { C } & 7.5070990000 & -0.7352990000 & 0.0000000000 \\ \text { H } & 7.5395030000 & -2.8816740000 & 0.0000000000 \\ \text { H } & 8.5866580000 & -0.6443890000 & 0.0000000000\end{array}$

\begin{tabular}{|c|c|c|c|}
\hline \multicolumn{4}{|c|}{ syn-IDBT-sulfone (6) } \\
\hline \multicolumn{3}{|c|}{ Zero-point correction $=$} & 293669 (Hartree/Particle) \\
\hline \multicolumn{3}{|c|}{ Thermal correction to Energy= } & 0.316214 \\
\hline \multicolumn{3}{|c|}{ Thermal correction to Enthalpy= } & 0.317158 \\
\hline \multicolumn{3}{|c|}{ Thermal correction to Gibbs Free Energy } & 0.241323 \\
\hline \multicolumn{4}{|c|}{ Sum of electronic and zero-point Energies= } \\
\hline $\mathrm{H}$ & -7.4283550000 & 2.7154620000 & 0.0000060000 \\
\hline $\mathrm{H}$ & -7.3050770000 & 0.2245250000 & 0.0000060000 \\
\hline $\mathrm{C}$ & -6.4643230000 & 2.2208310000 & 0.0000040000 \\
\hline $\mathrm{C}$ & -6.4030190000 & 0.8246870000 & 0.0000040000 \\
\hline $\mathrm{H}$ & -5.3572340000 & 4.0636520000 & 0.0000010000 \\
\hline $\mathrm{O}$ & -5.3108340000 & -2.1501520000 & -1.2606670000 \\
\hline $\mathrm{O}$ & -5.3108280000 & -2.1501530000 & 1.2606690000 \\
\hline $\mathrm{C}$ & -5.2914510000 & 2.9819560000 & 0.0000010000 \\
\hline $\mathrm{C}$ & -5.1552650000 & 0.2324390000 & 0.0000010000 \\
\hline $\mathrm{S}$ & -4.8539310000 & -1.5602150000 & 0.0000000000 \\
\hline $\mathrm{C}$ & -4.0405680000 & 2.3719600000 & -0.0000020000 \\
\hline $\mathrm{C}$ & -3.9554900000 & 0.9729500000 & -0.0000020000 \\
\hline $\mathrm{H}$ & -3.1436250000 & 2.9782500000 & -0.0000050000 \\
\hline $\mathrm{C}$ & -3.0957390000 & -1.2872070000 & -0.0000040000 \\
\hline $\mathrm{C}$ & -2.7848020000 & 0.1170310000 & -0.0000040000 \\
\hline $\mathrm{C}$ & -1.9578650000 & -2.0461040000 & -0.0000100000 \\
\hline $\mathrm{H}$ & -1.8858990000 & -3.1240450000 & -0.0000120000 \\
\hline $\mathrm{C}$ & -1.3845660000 & 0.2369970000 & -0.0000090000 \\
\hline $\mathrm{H}$ & -0.8934970000 & 2.3636770000 & -0.0000140000 \\
\hline $\mathrm{C}$ & -0.8481290000 & -1.1161370000 & 0.0000030000 \\
\hline $\mathrm{C}$ & -0.5107070000 & 1.3492330000 & -0.0000130000 \\
\hline $\mathrm{C}$ & 0.5107070000 & -1.3492330000 & 0.0000120000 \\
\hline $\mathrm{C}$ & 0.8481290000 & 1.1161370000 & -0.0000040000 \\
\hline $\mathrm{H}$ & 0.8934970000 & -2.3636770000 & 0.0000130000 \\
\hline $\mathrm{C}$ & 1.3845660000 & -0.2369970000 & 0.0000080000 \\
\hline $\mathrm{H}$ & 1.8858990000 & 3.1240450000 & 0.0000090000 \\
\hline $\mathrm{C}$ & 1.9578650000 & 2.0461040000 & 0.0000080000 \\
\hline $\mathrm{C}$ & 2.7848020000 & -0.1170310000 & 0.0000030000 \\
\hline $\mathrm{C}$ & 3.0957390000 & 1.2872070000 & 0.0000030000 \\
\hline $\mathrm{H}$ & 3.1436250000 & -2.9782500000 & 0.0000040000 \\
\hline
\end{tabular}




$\begin{array}{llll}\mathrm{C} & 3.9554900000 & -0.9729500000 & 0.0000010000 \\ \mathrm{C} & 4.0405680000 & -2.3719600000 & 0.0000020000 \\ \mathrm{~S} & 4.8539310000 & 1.5602150000 & 0.0000000000 \\ \mathrm{C} & 5.1552650000 & -0.2324390000 & -0.0000010000 \\ \mathrm{C} & 5.2914510000 & -2.9819560000 & 0.0000000000 \\ \mathrm{O} & 5.3108290000 & 2.1501530000 & -1.2606690000 \\ \mathrm{O} & 5.3108330000 & 2.1501520000 & 1.2606680000 \\ \mathrm{H} & 5.3572340000 & -4.0636520000 & 0.0000000000 \\ \mathrm{C} & 6.4030190000 & -0.8246870000 & -0.0000030000 \\ \mathrm{C} & 6.4643230000 & -2.2208310000 & -0.0000030000 \\ \mathrm{H} & 7.3050770000 & -0.2245250000 & -0.0000050000 \\ \mathrm{H} & 7.4283550000 & -2.7154620000 & -0.0000050000\end{array}$

\section{anti-IDT}

Zero-point correction=

Thermal correction to Energy=
0.183298 (Hartree/Particle)

0.196273

0.197217

Thermal correction to Gibbs Free Energy $=\quad 0.143954$

Sum of electronic and zero-point Energies $=\quad-1410.849992$ 


$\begin{array}{rrrr}\mathrm{H} & 2.2393570000 & 5.1295720000 & 0.0000000000 \\ \mathrm{H} & 2.3955180000 & -0.8233690000 & 0.0000000000 \\ \mathrm{H} & 2.9971940000 & 1.9723270000 & 0.0000000000\end{array}$

\begin{tabular}{|c|c|c|c|}
\hline \multicolumn{4}{|c|}{ syn-IDT } \\
\hline \multicolumn{3}{|c|}{ Zero-point correction $=$} & 0.183355 (Hartree/Particle) \\
\hline \multicolumn{3}{|c|}{ Thermal correction to Energy= } & 0.196302 \\
\hline \multicolumn{3}{|c|}{ Thermal correction to Enthalpy= } & 0.197247 \\
\hline \multicolumn{3}{|c|}{ Thermal correction to Gibbs Free Energy } & 0.144083 \\
\hline \multicolumn{4}{|c|}{ Sum of electronic and zero-point Energies $=$} \\
\hline $\mathrm{H}$ & -2.7653050000 & -2.2924930000 & 0.0000000000 \\
\hline $\mathrm{H}$ & -2.6067720000 & 3.6700130000 & 0.0000000000 \\
\hline $\mathrm{H}$ & -2.4680710000 & 0.5582100000 & 0.0000000000 \\
\hline $\mathrm{C}$ & -1.6874200000 & -2.2051470000 & 0.0000000000 \\
\hline $\mathrm{C}$ & -1.5350130000 & 3.8191340000 & 0.0000000000 \\
\hline $\mathrm{H}$ & -1.4568800000 & 6.0256140000 & 0.0000000000 \\
\hline $\mathrm{C}$ & -1.4048570000 & 0.3379260000 & 0.0000000000 \\
\hline $\mathrm{C}$ & -0.9600550000 & 5.0672500000 & 0.0000000000 \\
\hline $\mathrm{C}$ & -0.9600550000 & -1.0176220000 & 0.0000000000 \\
\hline S & -0.7833760000 & -5.0164130000 & 0.0000000000 \\
\hline $\mathrm{C}$ & -0.7480980000 & -3.2818890000 & 0.0000000000 \\
\hline $\mathrm{C}$ & -0.5626310000 & 2.7902320000 & 0.0000000000 \\
\hline $\mathrm{C}$ & -0.4680090000 & 1.3334880000 & 0.0000000000 \\
\hline $\mathrm{C}$ & 0.4680090000 & -1.3334880000 & 0.0000000000 \\
\hline $\mathrm{C}$ & 0.5626310000 & -2.7902320000 & 0.0000000000 \\
\hline $\mathrm{C}$ & 0.7480980000 & 3.2818890000 & 0.0000000000 \\
\hline S & 0.7833760000 & 5.0164130000 & 0.0000000000 \\
\hline $\mathrm{C}$ & 0.9600550000 & -5.0672500000 & 0.0000000000 \\
\hline $\mathrm{C}$ & 0.9600550000 & 1.0176220000 & 0.0000000000 \\
\hline $\mathrm{C}$ & 1.4048570000 & -0.3379260000 & 0.0000000000 \\
\hline $\mathrm{H}$ & 1.4568800000 & -6.0256140000 & 0.0000000000 \\
\hline $\mathrm{C}$ & 1.5350130000 & -3.8191340000 & 0.0000000000 \\
\hline $\mathrm{C}$ & 1.6874200000 & 2.2051470000 & 0.0000000000 \\
\hline $\mathrm{H}$ & 2.4680710000 & -0.5582100000 & 0.0000000000 \\
\hline $\mathrm{H}$ & 2.6067720000 & -3.6700130000 & 0.0000000000 \\
\hline $\mathrm{H}$ & 2.7653050000 & 2.2924930000 & 0.0000000000 \\
\hline
\end{tabular}

\section{anti-IDT-sulfone}

Zero-point correction $=$

Thermal correction to Energy=

0.183300 (Hartree/Particle)

Thermal correction to Enthalpy=

0.196273

0.197217 
$\begin{array}{lc}\text { Thermal correction to Gibbs Free Energy }= & 0.142964 \\ \text { Sum of electronic and zero-point Energies }= & -1410.849990\end{array}$

$\begin{array}{lrrr}\mathrm{H} & -3.2707130000 & 1.6703290000 & 0.0000000000 \\ \mathrm{H} & -2.8320730000 & 4.8257230000 & 0.0000000000 \\ \mathrm{H} & -2.2995320000 & -1.0858680000 & 0.0000000000 \\ \mathrm{C} & -2.2005550000 & 1.8216340000 & 0.0000000000 \\ \mathrm{C} & -1.8220110000 & 4.4335320000 & 0.0000000000 \\ \mathrm{O} & -1.5275260000 & -4.4583190000 & -1.2616400000 \\ \mathrm{O} & -1.5275260000 & -4.4583190000 & 1.2616400000 \\ \mathrm{C} & -1.5275260000 & 3.0112940000 & 0.0000000000 \\ \mathrm{C} & -1.3300870000 & -0.5997800000 & 0.0000000000 \\ \mathrm{C} & -1.2012990000 & 0.7579550000 & 0.0000000000 \\ \mathrm{~S} & -0.8070740000 & -4.2731500000 & 0.0000000000 \\ \mathrm{C} & -0.7322510000 & 5.2189140000 & 0.0000000000 \\ \mathrm{H} & -0.6653900000 & 6.2970170000 & 0.0000000000 \\ \mathrm{C} & -0.1312640000 & -1.3841590000 & 0.0000000000 \\ \mathrm{C} & -0.1004370000 & 2.7330210000 & 0.0000000000 \\ \mathrm{C} & 0.1004370000 & -2.7330210000 & 0.0000000000 \\ \mathrm{C} & 0.1312640000 & 1.3841590000 & 0.0000000000 \\ \mathrm{H} & 0.6653900000 & -6.2970170000 & 0.0000000000 \\ \mathrm{C} & 0.7322510000 & -5.2189140000 & 0.0000000000 \\ \mathrm{~S} & 0.8070740000 & 4.2731500000 & 0.0000000000 \\ \mathrm{C} & 1.2012990000 & -0.7579550000 & 0.0000000000 \\ \mathrm{C} & 1.3300870000 & 0.5997800000 & 0.0000000000 \\ \mathrm{O} & 1.5275260000 & 4.4583190000 & -1.2616400000 \\ \mathrm{O} & 1.5275260000 & 4.4583190000 & 1.2616400000 \\ \mathrm{C} & 1.5275260000 & -3.0112940000 & 0.0000000000 \\ \mathrm{C} & 1.8220110000 & -4.4335320000 & 0.0000000000 \\ \mathrm{H} & 2.2005550000 & -1.8216340000 & 0.0000000000 \\ \mathrm{H} & 2.2995320000 & 1.0858680000 & 0.0000000000 \\ \mathrm{H} & 2.8320730000 & -4.8257230000 & 0.0000000000 \\ & 3.2707130000 & -1.6703290000 & 0.0000000000\end{array}$

syn-IDT-sulfone

Zero-point correction=

Thermal correction to Energy=

0.183352 (Hartree/Particle)

Thermal correction to Enthalpy=

0.196302

0.197246

Thermal correction to Gibbs Free Energy=

0.144074

Sum of electronic and zero-point Energies=

$-1410.852466$
$\mathrm{H} \quad-2.9974210000$
$-3.1920250000$
0.0000000000
$\mathrm{H} \quad-2.5301400000$
2.6514700000
0.0000000000 


$\begin{array}{lrrr}\mathrm{H} & -2.5207810000 & -0.2584490000 & 0.0000000000 \\ \mathrm{H} & -2.2645050000 & -5.7074210000 & 0.0000000000 \\ \mathrm{C} & -1.9691160000 & -3.5331990000 & 0.0000000000 \\ \mathrm{C} & -1.6265860000 & -4.8351550000 & 0.0000000000 \\ \mathrm{C} & -1.4714450000 & 2.4368130000 & 0.0000000000 \\ \mathrm{C} & -1.4425210000 & -0.1365370000 & 0.0000000000 \\ \mathrm{C} & -0.8678590000 & 1.1089430000 & 0.0000000000 \\ \mathrm{C} & -0.8329970000 & -2.6395520000 & 0.0000000000 \\ \mathrm{O} & -0.5831520000 & 5.7011430000 & -1.2622700000 \\ \mathrm{O} & -0.5831520000 & 5.7011430000 & 1.2622700000 \\ \mathrm{C} & -0.5831520000 & -1.2693760000 & 0.0000000000 \\ \mathrm{C} & -0.4403490000 & 3.3237980000 & 0.0000000000 \\ \mathrm{~S} & -0.1681710000 & 5.0869040000 & 0.0000000000 \\ \mathrm{~S} & 0.1681710000 & -5.0869040000 & 0.0000000000 \\ \mathrm{C} & 0.4403490000 & -3.3237980000 & 0.0000000000 \\ \mathrm{O} & 0.5831520000 & -5.7011430000 & -1.2622700000 \\ \mathrm{O} & 0.5831520000 & -5.7011430000 & 1.2622700000 \\ \mathrm{C} & 0.5831520000 & 1.2693760000 & 0.0000000000 \\ \mathrm{C} & 0.8329970000 & 2.6395520000 & 0.0000000000 \\ \mathrm{C} & 0.8678590000 & -1.1089430000 & 0.0000000000 \\ \mathrm{C} & 1.4425210000 & 0.1365370000 & 0.0000000000 \\ \mathrm{C} & 1.4714450000 & -2.4368130000 & 0.0000000000 \\ \mathrm{C} & 1.6265860000 & 4.8351550000 & 0.0000000000 \\ \mathrm{C} & 1.9691160000 & 3.5331990000 & 0.0000000000 \\ \mathrm{H} & 2.2645050000 & 5.7074210000 & 0.0000000000 \\ \mathrm{H} & 2.5207810000 & 0.2584490000 & 0.0000000000 \\ \mathrm{H} & 2.5301400000 & -2.6514700000 & 0.0000000000 \\ \mathrm{H} & 2.9974210000 & 3.1920250000 & 0.0000000000\end{array}$

\section{PDA-Standard}

Zero-point correction $=$

Thermal correction to Energy=

0.320129 (Hartree/Particle)

Thermal correction to Enthalpy=

0.338424

0.339368

Thermal correction to Gibbs Free Energy $=\quad 0.273840$

Sum of electronic and zero-point Energies $=\quad-1075.284484$
$\mathrm{H} \quad-7.2773800000 \quad 0.9066240000 \quad-0.0000050000$
$\mathrm{H} \quad-6.9131660000 \quad-1.7608810000 \quad-0.0000070000$
$\mathrm{H} \quad-6.2075970000 \quad 3.1148090000 \quad-0.0000010000$
C $\quad-6.1954600000 \quad 0.9841280000 \quad-0.0000030000$
C $\quad-5.8492500000 \quad-1.5482230000 \quad-0.0000050000$
C $\quad-5.5883320000 \quad 2.2245160000 \quad-0.0000010000$
C $\quad-5.3945160000 \quad-0.1977700000 \quad-0.0000030000$ 


$\begin{array}{lrrr}\mathrm{H} & -5.3021420000 & -3.6099510000 & -0.0000060000 \\ \mathrm{C} & -4.9371260000 & -2.5888840000 & -0.0000040000 \\ \mathrm{C} & -4.1735680000 & 2.3852410000 & 0.0000010000 \\ \mathrm{C} & -4.0136820000 & -0.0117290000 & -0.0000010000 \\ \mathrm{H} & -3.7544210000 & 3.3857060000 & 0.0000030000 \\ \mathrm{C} & -3.5337090000 & -2.3705570000 & -0.0000020000 \\ \mathrm{C} & -3.3710140000 & 1.2609710000 & 0.0000020000 \\ \mathrm{C} & -3.0599310000 & -1.0715750000 & 0.0000000000 \\ \mathrm{H} & -2.8621710000 & -3.2220680000 & -0.0000020000 \\ \mathrm{C} & -1.9441730000 & 0.9626600000 & 0.0000040000 \\ \mathrm{C} & -1.7602950000 & -0.4165070000 & 0.0000020000 \\ \mathrm{C} & -0.6395170000 & 1.6329880000 & 0.0000070000 \\ \mathrm{H} & -0.4852010000 & 2.7043930000 & 0.0000080000 \\ \mathrm{C} & -0.3222170000 & -0.6612310000 & 0.0000040000 \\ \mathrm{C} & 0.3222170000 & 0.6612310000 & 0.0000040000 \\ \mathrm{H} & 0.4852010000 & -2.7043930000 & 0.0000080000 \\ \mathrm{C} & 0.6395170000 & -1.6329880000 & 0.0000070000 \\ \mathrm{C} & 1.7602950000 & 0.4165070000 & 0.0000020000 \\ \mathrm{C} & 1.9441730000 & -0.9626600000 & 0.0000040000 \\ \mathrm{H} & 2.8621710000 & 3.2220680000 & -0.0000020000 \\ \mathrm{C} & 3.0599310000 & 1.0715750000 & 0.0000000000 \\ \mathrm{C} & 3.3710140000 & -1.2609710000 & 0.0000020000 \\ \mathrm{C} & 3.5337090000 & 2.3705570000 & -0.0000020000 \\ \mathrm{H} & 3.7544210000 & -3.3857060000 & 0.0000030000 \\ \mathrm{C} & 4.0136820000 & 0.0117290000 & -0.0000010000 \\ \mathrm{C} & 4.1735680000 & -2.3852410000 & 0.0000010000 \\ \mathrm{C} & 4.9371260000 & 2.5888840000 & -0.0000040000 \\ \mathrm{H} & 5.3021420000 & 3.6099510000 & -0.0000060000 \\ \mathrm{C} & 5.3945160000 & 0.1977700000 & -0.0000030000 \\ \mathrm{C} & 5.5883320000 & -2.2245160000 & -0.0000010000 \\ \mathrm{C} & 5.8492500000 & 1.5482230000 & -0.0000050000 \\ \mathrm{C} & 6.1954600000 & -0.9841280000 & -0.0000030000 \\ \mathrm{H} & 6.2075970000 & -3.1148090000 & -0.0000010000 \\ \mathrm{H} & 6.9131660000 & 1.7608810000 & -0.0000070000 \\ \mathrm{H} & 7.2773800000 & -0.9066240000 & -0.0000050000\end{array}$

\section{PDA-Bond-flipped}

Zero-point correction $=$

Thermal correction to Energy=

Thermal correction to Enthalpy=

Thermal correction to Gibbs Free Energy=

Sum of electronic and zero-point Energies=

Sum of electronic and thermal Energies=

Sum of electronic and thermal Enthalpies=

Sum of electronic and thermal Free Energies=
0.321003 (Hartree/Particle)

0.339015

0.339959

0.275158

$-1075.300781$

$-1075.282769$

$-1075.281825$

$-1075.346626$ 


$\begin{array}{rrrr}\mathrm{H} & -7.2212060000 & 0.8393980000 & 0.0000010000 \\ \mathrm{H} & -6.8162160000 & -1.8036280000 & 0.0000010000 \\ \mathrm{H} & -6.2055850000 & 3.0700410000 & 0.0000010000 \\ \mathrm{C} & -6.1412190000 & 0.9401090000 & 0.0000010000 \\ \mathrm{C} & -5.7548260000 & -1.5789410000 & 0.0000010000 \\ \mathrm{C} & -5.5628090000 & 2.1964010000 & 0.0000010000 \\ \mathrm{C} & -5.3234120000 & -0.2239320000 & 0.0000000000 \\ \mathrm{H} & -5.1897960000 & -3.6349600000 & 0.0000000000 \\ \mathrm{C} & -4.8323190000 & -2.6110910000 & 0.0000000000 \\ \mathrm{C} & -4.1587590000 & 2.3907150000 & 0.0000000000 \\ \mathrm{C} & -3.9330210000 & -0.0128830000 & 0.0000000000 \\ \mathrm{H} & -3.7561520000 & 3.3970910000 & 0.0000000000 \\ \mathrm{C} & -3.4355170000 & -2.3807090000 & 0.0000000000 \\ \mathrm{C} & -3.3318880000 & 1.2830800000 & 0.0000000000 \\ \mathrm{C} & -2.9778880000 & -1.0753780000 & 0.0000000000 \\ \mathrm{H} & -2.7504660000 & -3.2204310000 & 0.0000000000 \\ \mathrm{C} & -1.8953430000 & 1.0556230000 & -0.0000010000 \\ \mathrm{C} & -1.6733430000 & -0.4395710000 & -0.0000010000 \\ \mathrm{C} & -0.6979220000 & 1.7017140000 & -0.0000010000 \\ \mathrm{H} & -0.5241060000 & 2.7680520000 & 0.0000000000 \\ \mathrm{C} & -0.3298590000 & -0.6616700000 & -0.0000010000 \\ \mathrm{C} & 0.3298590000 & 0.6616700000 & -0.0000010000 \\ \mathrm{H} & 0.5241060000 & -2.7680520000 & 0.0000000000 \\ \mathrm{C} & 0.6979220000 & -1.7017140000 & -0.0000010000 \\ \mathrm{C} & 1.6733430000 & 0.4395710000 & -0.0000010000 \\ \mathrm{C} & 1.8953430000 & -1.0556230000 & -0.0000010000 \\ \mathrm{H} & 2.7504660000 & 3.2204310000 & 0.0000000000 \\ \mathrm{C} & 2.9778880000 & 1.0753780000 & 0.0000000000 \\ \mathrm{C} & 3.3318880000 & -1.2830800000 & 0.0000000000 \\ \mathrm{C} & 3.4355170000 & 2.3807090000 & 0.0000000000 \\ \mathrm{H} & 3.7561520000 & -3.3970910000 & 0.0000000000 \\ \mathrm{C} & 3.9330210000 & 0.0128830000 & 0.0000000000 \\ \mathrm{C} & 4.1587590000 & -2.3907150000 & 0.0000000000 \\ \mathrm{C} & 4.8323190000 & 2.6110910000 & 0.0000000000 \\ \mathrm{H} & 5.1897960000 & 3.6349600000 & 0.0000000000 \\ \mathrm{C} & 5.3234120000 & 0.2239320000 & 0.0000000000 \\ \mathrm{C} & 5.5628090000 & -2.1964010000 & 0.0000010000 \\ \mathrm{H} & 6.7548260000 & 1.5789410000 & 0.0000010000 \\ \mathrm{H} & 6.2055850000 & -3.0700410000 & 0.0000010000 \\ \mathrm{H} & -2212060000 & -0.8393980000 & 0.0000010000\end{array}$




\subsection{Calculated HOMO/LUMO Energy Levels with TD-DFT Calculations for Predicted UV-Vis Spectra}

The same geometries used above for the NICS-XY scans were used to predict UV-vis spectra and calculate optical energy gaps for the IDBTs and IDBT sulfones. Optical values were calculated with TD-B3LYP/6-311++G** for the IDBTs and IDBT-sulfones, and with TD-B3LYP/6-311+G* for the IIDBTs and IIDBT-sulfones. HOMO-LUMO levels were generated using B3LYP/6$311++\mathrm{G}^{* *}$ for the IDBTs and IDBT-sulfones, and using B3LYP/6-311+G* for the IIDBTs and IIDBT-sulfones.

Table S1. Calculated optical data and HOMO-LUMO energy gaps for compounds 1-8.

\begin{tabular}{cccccccc}
\hline & $\begin{array}{c}\lambda_{\max } \\
(\mathrm{nm})\end{array}$ & $\mathrm{f}$ & configuration & $\begin{array}{c}\text { Optical } \\
\text { Gap }(\mathrm{eV})\end{array}$ & $\begin{array}{c}\text { HOMO } \\
(\mathrm{eV})\end{array}$ & $\begin{array}{c}\text { LUMO } \\
(\mathrm{eV})\end{array}$ & $\begin{array}{c}\text { Egap } \\
(\mathrm{eV})\end{array}$ \\
\hline anti-IDBT (1) & 597 & 0.3685 & HOMO-1 $\rightarrow$ LUMO (93\%) & 2.05 & -5.297 & -3.336 & 1.961 \\
anti-IDBT-S (5) & 550 & 0.1528 & HOMO-1 $\rightarrow$ LUMO (75\%) & 2.20 & -6.179 & -4.339 & 1.840 \\
syn-IDBT (2) & 608 & 0.5578 & HOMO-1 $\rightarrow$ LUMO (94\%) & 1.99 & -5.292 & -3.455 & 1.837 \\
syn-IDBT-S (6) & 603 & 0.7178 & HOMO $\rightarrow$ LUMO (99\%) & 2.04 & -6.347 & -4.393 & 1.954 \\
anti-IIDBT (3) & 702 & 0.5576 & HOMO $\rightarrow$ LUMO (95\%) & 1.78 & -4.983 & -3.378 & 1.605 \\
anti-IIDBT-S (7) & 639 & 0.4487 & HOMO $\rightarrow$ LUMO (93\%) & 1.94 & -6.198 & -4.387 & 1.811 \\
syn-IIDBT (4) & 730 & 0.7576 & HOMO $\rightarrow$ LUMO (100\%) & 1.71 & -5.100 & -3.572 & 1.528 \\
syn-IIDBT-S (8) & 716 & 0.8410 & HOMO $\rightarrow$ LUMO (100\%) & 1.75 & -5.888 & -4.368 & 1.520 \\
\hline
\end{tabular}

\section{anti-IIDBT (3)}

Zero-point correction=

Thermal correction to Energy=

Thermal correction to Enthalpy=

Thermal correction to Gibbs Free Energy=

Sum of electronic and zero-point Energies=

\subsection{6 (Hartree/Particle)}

0.344386

0.345330

0.272772

$-1871.733601$

$\begin{array}{rrrc}\mathrm{H} & -9.5125540000 & -1.1169460000 & 1.7421150000 \\ \mathrm{H} & -8.6458070000 & 0.1993580000 & 3.6537040000 \\ \mathrm{C} & -8.5662770000 & -0.5940130000 & 1.6519960000 \\ \mathrm{H} & -8.2326090000 & -1.2479210000 & -0.3761170000 \\ \mathrm{C} & -8.0747920000 & 0.1509030000 & 2.7323870000\end{array}$




\begin{tabular}{|c|c|c|c|}
\hline $\mathrm{C}$ & -7.8511110000 & -0.6708640000 & 000 \\
\hline $\mathrm{C}$ & -6.8650350000 & 0.8275090000 & 270000 \\
\hline $\mathrm{C}$ & -6.6394540000 & 0.0084530000 & 80000 \\
\hline $\mathrm{H}$ & -6.4917300000 & 1.4022090000 & \\
\hline & -6.1243450000 & 0.7673650000 & 1. \\
\hline 5 & 950000 & 5450000 & -1. \\
\hline $\mathrm{C}$ & -4.8606620000 & 1.3715880000 & 56000 \\
\hline $\mathrm{C}$ & -4.4509890000 & 1.0620710000 & -0.2 \\
\hline $\mathrm{H}$ & 390000 & 2.60722 & \\
\hline$C$ & 70000 & 2560000 & 1. \\
\hline $\mathrm{C}$ & 40000 & 1.692271 & \\
\hline 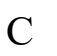 & 520000 & 2.41221 & 0. \\
\hline $\mathrm{H}$ & 50000 & 1.2 & \\
\hline $\mathrm{C}$ & 000 & 1.7 & \\
\hline $\mathrm{C}$ & 0000 & 3.1 & 0.8 \\
\hline $\mathrm{H}$ & -1.4 & 3.6 & 1.7 \\
\hline $\mathrm{C}$ & 370000 & $2.4^{\prime}$ & -1 \\
\hline $\mathrm{C}$ & -0.8 & & \\
\hline $\mathrm{H}$ & 0000 & 000 & -3 \\
\hline $\mathrm{C}$ & -0.2 & 2.5 & -2 \\
\hline $\mathrm{C}$ & 0.4 & 3.9 & -0 \\
\hline $\mathrm{H}$ & 000 & 4.4 & 0.6 \\
\hline $\mathrm{C}$ & 0000 & $3.25^{\circ}$ & -2.5 \\
\hline $\mathrm{C}$ & 00000 & $3.9^{\prime}$ & -1.3 \\
\hline $\mathrm{C}$ & 1.9 & & \\
\hline $\mathrm{H}$ & 60000 & 3.0 & -4 \\
\hline $\mathrm{C}$ & 0000 & 4.6 & -1 \\
\hline $\mathrm{C}$ & 90000 & 4.25 & -2. \\
\hline S & 810000 & 5.6279680 & -0.7 \\
\hline $\mathrm{C}$ & 000 & 4.9 & 49000 \\
\hline $\mathrm{H}$ & 0000 & 4.26 & 376000 \\
\hline $\mathrm{C}$ & 400000 & & \\
\hline $\mathrm{C}$ & 4.91 & 0030000 & -4 \\
\hline $\mathrm{C}$ & 0000 & 6.3 & -2.2 \\
\hline $\mathrm{C}$ & 780000 & 5.51 & -4.533701000 \\
\hline $\mathrm{H}$ & 6.2826950000 & 6.9174340000 & -1.42519700 \\
\hline $\mathrm{C}$ & 6.6163630000 & 6.2635260000 & -3.453310000 \\
\hline $\mathrm{H}$ & 6.6958940000 & 5.4701550000 & -5.45501900 \\
\hline $\mathrm{H}$ & 7.5626400000 & 6.7864590000 & -3.54342900 \\
\hline
\end{tabular}

anti-IIDBT-sulfone (7)

Zero-point correction=

Thermal correction to Energy=

0.339841 (Hartree/Particle)

0.365195 


$$
\begin{array}{lc}
\text { Thermal correction to Enthalpy= } & 0.366139 \\
\text { Thermal correction to Gibbs Free Energy }= & 0.283946 \\
\text { Sum of electronic and zero-point Energies }= & -2172.523676
\end{array}
$$

\begin{tabular}{|c|c|c|c|}
\hline & 0.7904070000 & -2.5882160000 & 000 \\
\hline & 1.2079160000 & -0.3307170000 & 162780000 \\
\hline & 1.4921500000 & -1.9274980000 & -4.7366780000 \\
\hline & 1.7247320000 & -0.6553950000 & 120000 \\
\hline & 1.9533120000 & -3.3560940000 & -6.28 \\
\hline & 2.0640750000 & 2.3740730000 & 0000 \\
\hline & 2.1476690000 & -2.3635350000 & -5.8902900000 \\
\hline & 2.6276180000 & 0.1939 & -4 \\
\hline & $2.7482^{\prime}$ & 2.4912 & -3.5 \\
\hline & 0000 & 000 & -4.5 \\
\hline & 3.0549270000 & -1.526 & 000 \\
\hline & 3.0661390000 & 5.0778 & -2.2 \\
\hline C & 3.2732890000 & -0.26 & -6.01 \\
\hline & 3.5315670 & 3.6 & -3.7 \\
\hline H & 3.5710 & -1.8 & -7 \\
\hline & 3.6423540000 & 4.87 & -3.13 \\
\hline D & 3.92 & & -7.5 \\
\hline & & & -5.3 \\
\hline $\mathrm{O}$ & 4.0125570000 & 10.969 & -1.88 \\
\hline $\mathrm{H}$ & 4.04 & 000 & -2.08 \\
\hline $\mathrm{C}$ & 4.31 & 3.4 & -4.9 \\
\hline & & & \\
\hline$C$ & 4.51 & 5.8 & -3. \\
\hline $\mathrm{C}$ & 4.62 & 7.1 & -2.9 \\
\hline $\mathrm{C}$ & 5.1798270000 & 4.39 & -5.49 \\
\hline & 5.29 & 5.62 & -4.83 \\
\hline $\mathrm{S}$ & 5.406 & 10.5 & -1.7 \\
\hline $\mathrm{C}$ & 5.49 & 8.0 & -3.48 \\
\hline $\mathrm{H}$ & 5.76 & 4.2 & -6.3 \\
\hline O & 5.7970750000 & 000 & -6.58 \\
\hline $\mathrm{C}$ & 5.825 & 9.38 & -3.078 \\
\hline $\mathrm{O}$ & 5.8830250000 & 10.0459780000 & -0.4777660000 \\
\hline $\mathrm{C}$ & 6.1672780000 & 6.632 & -5.3335970000 \\
\hline $\mathrm{H}$ & 6.2386160000 & 13.3 & -1.031335000 \\
\hline $\mathrm{C}$ & 6.2780650000 & 7.84 & 520000 \\
\hline $\mathrm{C}$ & 6.5363430000 & 11.7737010000 & -2.4605220000 \\
\hline I & 6.7434930000 & 6.4266370000 & -6.231686000 \\
\hline $\mathrm{C}$ & 6.7547040000 & 13.0310590000 & -1.927899000 \\
\hline S & 6.7763630000 & 9.9499000000 & -3.970905000 \\
\hline
\end{tabular}




$\begin{array}{llcl}\text { C } & 7.0613590000 & 9.0133090000 & -4.9609740000 \\ \text { C } & 7.1820140000 & 11.3105470000 & -3.6187950000 \\ \text { C } & 7.6619630000 & 13.8680470000 & -2.5829490000 \\ \text { H } & 7.7455570000 & 9.1304380000 & -5.7909850000 \\ \text { H } & 7.8563190000 & 14.8606060000 & -2.1916680000 \\ \text { C } & 8.0849000000 & 12.1599070000 & -4.2599270000 \\ \text { C } & 8.3174820000 & 13.4320100000 & -3.7365610000 \\ \text { H } & 8.6017160000 & 11.8352280000 & -5.1569610000 \\ \text { H } & 9.0192240000 & 14.0927270000 & -4.2345380000\end{array}$

syn-IIDBT (4)

Zero-point correction=

Thermal correction to Energy=

Thermal correction to Enthalpy=

0.323198 (Hartree/Particle)

Thermal correction to Gibbs Free Energy=

Sum of electronic and zero-point Energies=

0.344397

0.345341

0.272835

$-1871.734022$

$\begin{array}{lrrr}\mathrm{H} & -8.5812330000 & -1.4340420000 & 2.1361090000 \\ \mathrm{~S} & -7.6803850000 & 0.5772850000 & 0.0681060000 \\ \mathrm{C} & -7.5261770000 & -1.2063080000 & 2.2463990000 \\ \mathrm{H} & -7.2470360000 & -2.5240180000 & 3.9154730000 \\ \mathrm{C} & -6.8997280000 & -0.3000220000 & 1.3942560000 \\ \mathrm{C} & -6.7725680000 & -1.8159420000 & 3.2442980000 \\ \mathrm{H} & -6.3105990000 & 2.7649410000 & -2.0394960000 \\ \mathrm{C} & -6.1615990000 & 1.3588980000 & -0.3128840000 \\ \mathrm{C} & -5.7180480000 & 2.2948280000 & -1.2660310000 \\ \mathrm{C} & -5.5156500000 & 0.0121130000 & 1.5230940000 \\ \mathrm{C} & -5.4072020000 & -1.5235200000 & 3.3897660000 \\ \mathrm{C} & -5.1090210000 & 0.9640190000 & 0.5374080000 \\ \mathrm{H} & -4.8365800000 & -2.0094480000 & 4.1742710000 \\ \mathrm{C} & -4.7812320000 & -0.6220660000 & 2.5432110000 \\ \mathrm{C} & -4.3434290000 & 2.5082910000 & -1.0230830000 \\ \mathrm{C} & -3.9327220000 & 1.6803820000 & 0.1103350000 \\ \mathrm{H} & -3.7258030000 & -0.4051500000 & 2.6665600000 \\ \mathrm{H} & -3.7146970000 & 3.9443570000 & -2.5041520000 \\ \mathrm{C} & -3.4110560000 & 3.3282000000 & -1.6613630000 \\ \mathrm{C} & -2.6300110000 & 1.7147090000 & 0.5404190000 \\ \mathrm{H} & -2.2993730000 & 1.1096140000 & 1.3798480000 \\ \mathrm{C} & -2.0799730000 & 3.3689980000 & -1.2286890000 \\ \mathrm{C} & -1.6700740000 & 2.5494090000 & -0.1055150000 \\ \mathrm{H} & -1.4506740000 & 4.8087940000 & -2.7140520000 \\ \mathrm{C} & -1.1200360000 & 4.2036980000 & -1.8746230000 \\ \mathrm{C} & -0.3389920000 & 2.5902090000 & 0.3271600000\end{array}$




$\begin{array}{lrrr}\mathrm{H} & -0.0353500000 & 1.9740510000 & 1.1699480000 \\ \mathrm{H} & -0.0242460000 & 6.3235640000 & -4.0007590000 \\ \mathrm{C} & 0.1826740000 & 4.2380260000 & -1.4445390000 \\ \mathrm{C} & 0.5933820000 & 3.4101170000 & -0.3111210000 \\ \mathrm{C} & 1.0311830000 & 6.5404800000 & -3.8774100000 \\ \mathrm{H} & 1.0865300000 & 7.9278660000 & -5.5084670000 \\ \mathrm{C} & 1.3589740000 & 4.9543910000 & -1.8716110000 \\ \mathrm{C} & 1.6571520000 & 7.4419360000 & -4.7239630000 \\ \mathrm{C} & 1.7656010000 & 5.9062990000 & -2.8572940000 \\ \mathrm{C} & 1.9680020000 & 3.6235760000 & -0.0681750000 \\ \mathrm{C} & 2.4115520000 & 4.5595100000 & -1.0213200000 \\ \mathrm{H} & 2.5605530000 & 3.1534620000 & 0.7052880000 \\ \mathrm{C} & 3.0225190000 & 7.7343580000 & -4.5784950000 \\ \mathrm{C} & 3.1496790000 & 6.2184350000 & -2.7284570000 \\ \mathrm{H} & 3.4969860000 & 8.4424350000 & -5.2496690000 \\ \mathrm{C} & 3.7761280000 & 7.1247220000 & -3.5805980000 \\ \mathrm{~S} & 3.9303370000 & 5.3411250000 & -1.4023080000 \\ \mathrm{H} & 4.8311840000 & 7.3524560000 & -3.4703070000\end{array}$

syn-IIDBT-sulfone (8)

Zero-point correction=

Thermal correction to Energy=

0.340273 (Hartree/Particle)

Thermal correction to Enthalpy=

0.365517

0.366461

Thermal correction to Gibbs Free Energy $=\quad 0.284546$

Sum of electronic and zero-point Energies $=\quad-2172.526341$

$\begin{array}{lrrr}\mathrm{O} & 0.9799490000 & 1.4332070000 & -0.5345300000 \\ \mathrm{H} & 1.4923090000 & -1.5697660000 & 0.8772780000 \\ \mathrm{H} & 2.1228980000 & -3.8675570000 & 0.1340150000 \\ \mathrm{C} & 2.1711300000 & -1.7169130000 & 0.0444140000 \\ \mathrm{~S} & 2.3794920000 & 1.1049740000 & -0.2518740000 \\ \mathrm{C} & 2.5286140000 & -3.0015470000 & -0.3772050000 \\ \mathrm{C} & 2.7091060000 & -0.6407170000 & -0.6324510000 \\ \mathrm{O} & 2.9365410000 & 1.4557930000 & 1.0569280000 \\ \mathrm{C} & 3.4046530000 & -3.1728380000 & -1.4522670000 \\ \mathrm{C} & 3.4527560000 & 1.6228770000 & -1.5789430000 \\ \mathrm{C} & 3.5951490000 & -0.7813070000 & -1.7203660000 \\ \mathrm{H} & 3.6125480000 & 3.8134120000 & -1.8063720000 \\ \mathrm{H} & 3.6730830000 & -4.1752680000 & -1.7682560000 \\ \mathrm{C} & 3.8804910000 & 2.8147520000 & -2.1220160000 \\ \mathrm{C} & 3.9397570000 & -2.0772380000 & -2.1258850000 \\ \mathrm{C} & 4.0133930000 & 0.5012670000 & -2.2530640000 \\ \mathrm{H} & 4.6175470000 & -2.2333860000 & -2.9572310000\end{array}$




$\begin{array}{llll}\mathrm{C} & 4.7616410000 & 2.4684790000 & -3.2014420000 \\ \mathrm{C} & 4.8428330000 & 1.0111900000 & -3.2810240000 \\ \mathrm{H} & 5.4126830000 & 4.3369860000 & -4.0286630000 \\ \mathrm{C} & 5.4697020000 & 3.2531440000 & -4.0838200000 \\ \mathrm{C} & 5.6421770000 & 0.4198850000 & -4.2569300000 \\ \mathrm{H} & 5.7177770000 & -0.6603470000 & -4.3352690000 \\ \mathrm{C} & 6.2865510000 & 2.6569430000 & -5.0810550000 \\ \mathrm{C} & 6.3682820000 & 1.2095630000 & -5.1616850000 \\ \mathrm{H} & 6.9370560000 & 4.5268530000 & -5.9074700000 \\ \mathrm{C} & 7.0126560000 & 3.4466210000 & -5.9858100000 \\ \mathrm{C} & 7.1851310000 & 0.6133620000 & -6.1589200000 \\ \mathrm{H} & 7.2421500000 & -0.4704800000 & -6.2140770000 \\ \mathrm{C} & 7.8120010000 & 2.8553160000 & -6.9617150000 \\ \mathrm{C} & 7.8931920000 & 1.3980270000 & -7.0412980000 \\ \mathrm{H} & 8.0372850000 & 6.0998920000 & -7.2855100000 \\ \mathrm{C} & 8.6414400000 & 3.3652390000 & -7.9896760000 \\ \mathrm{C} & 8.7150760000 & 5.9437440000 & -8.1168550000 \\ \mathrm{C} & 8.7743430000 & 1.0517540000 & -8.1207240000 \\ \mathrm{H} & 8.9817500000 & 8.0417740000 & -8.4744840000 \\ \mathrm{H} & 9.0422850000 & 0.0530940000 & -8.4363680000 \\ \mathrm{C} & 9.0596840000 & 4.6478130000 & -8.5223740000 \\ \mathrm{C} & 9.2020780000 & 2.2436290000 & -8.6637970000 \\ \mathrm{C} & 9.2501800000 & 7.0393440000 & -8.7904730000 \\ \mathrm{O} & 9.7182940000 & 2.4107130000 & -11.2996670000 \\ \mathrm{C} & 9.9457270000 & 4.5072230000 & -9.6102890000 \\ \mathrm{C} & 10.1262190000 & 6.8680530000 & -9.8655350000 \\ \mathrm{~S} & 10.2753420000 & 2.7615320000 & -9.9908650000 \\ \mathrm{C} & 10.4837030000 & 5.5834190000 & -10.2871540000 \\ \mathrm{H} & 10.5319350000 & 7.7340630000 & -10.3767550000 \\ \mathrm{H} & 11.1625240000 & 5.4362720000 & -11.1200170000 \\ \mathrm{O} & 11.6748850000 & 2.4333000000 & -9.7082090000\end{array}$




\subsection{Clar Sextet-Effects in syn- and anti-IDBT Fused $s$-Indacenes}

Geometries for the dianion hydrocarbon reference compounds that are isoelectronic with the benzothiophene-fused $s$-indacenes (i.e., $s y n$-IDBT 2 and anti-IDBT 1) were optimized at B3LYP/6-311++G(d,p) using the Gaussian16 program. ${ }^{10}$ Vibrational frequency analyses verified the nature of the stationary points. Natural Lewis structure (NLS) analyses were performed at the same level of theory using the NBO 7.0 program $^{11}$ to identify the major Lewis structures of the dianion hydrocarbon references and illustrate the "Clar sextet-effect". The NLS procedure finds and prints out a representation of the best possible Lewis structure for a given molecular wavefunction. According to the recognized Lewis structure, atom numbers specified in the "LONE" string indicate the locations of lone pairs, and the pairs of atom numbers specified in the "BOND" string indicate the locations of single (S) and double (D) bonds. The NLS-recognized Lewis structures for the syn-IDBT dianion reference (Figure S24, note absence of Clar sextets in the suggested Lewis structure) and the anti-IDBT dianion reference (Figure S25, note two Clar sextets in the suggested Lewis structure) are shown below.
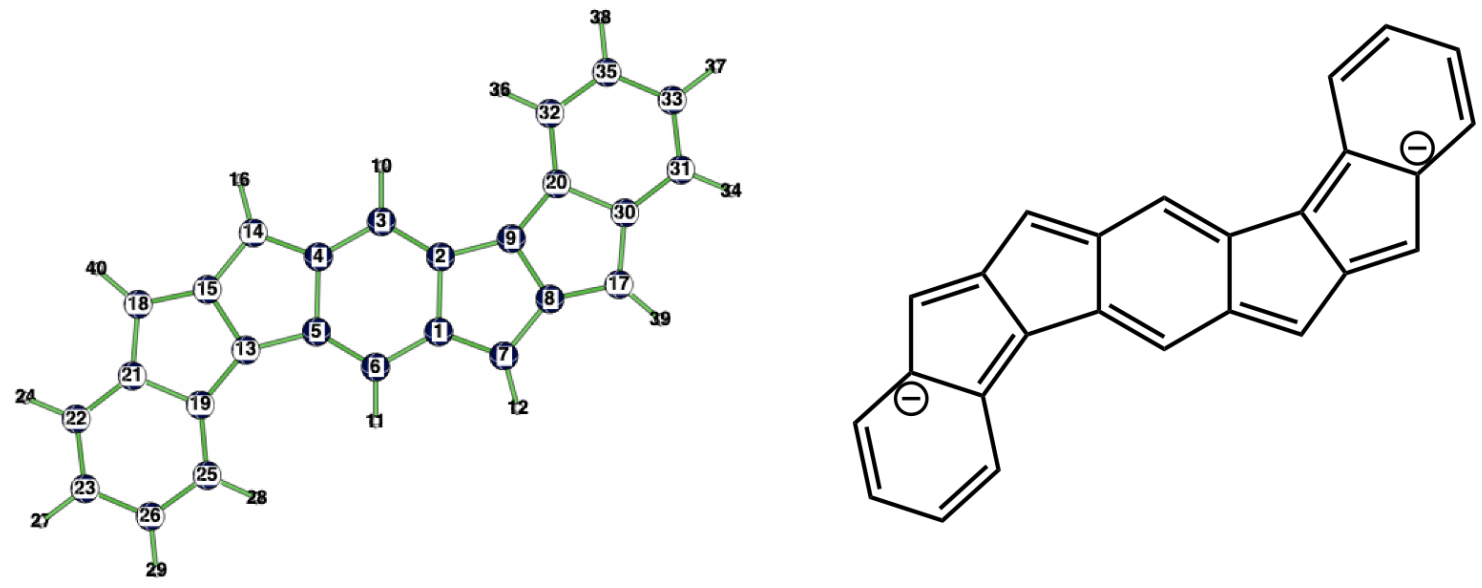

\section{LONE 211301 END}

BOND S 12 S 16 D 17 D 23 S 29 S 34 S 310 S 45 D 414 D 56 S 513 S 611 S 78 S 712 S $89 \mathrm{D} 817$ D $920 \mathrm{~S} 1315 \mathrm{D} 1319 \mathrm{~S} 1415 \mathrm{~S} 1416 \mathrm{D} 1518 \mathrm{~S} 1730$ S 1739 S 1821 S 1840 S 1921 S 1925 S 2030 S 2032 S 2122 D 2223 S 2224 S 2326 S 2327 D 2526 S 2528 S 2629 S 3031 D 3133 S 3134 D 3235 S 3236 S 3335 S 3337 S 3538 END

Figure S24. NLS output string, atom numbering, and Lewis structure for the isoelectronic dianion hydrocarbon reference structure of syn-IDBT 2. Note the absence of formal Clar sextets in the NLS-recognized Lewis structure. 

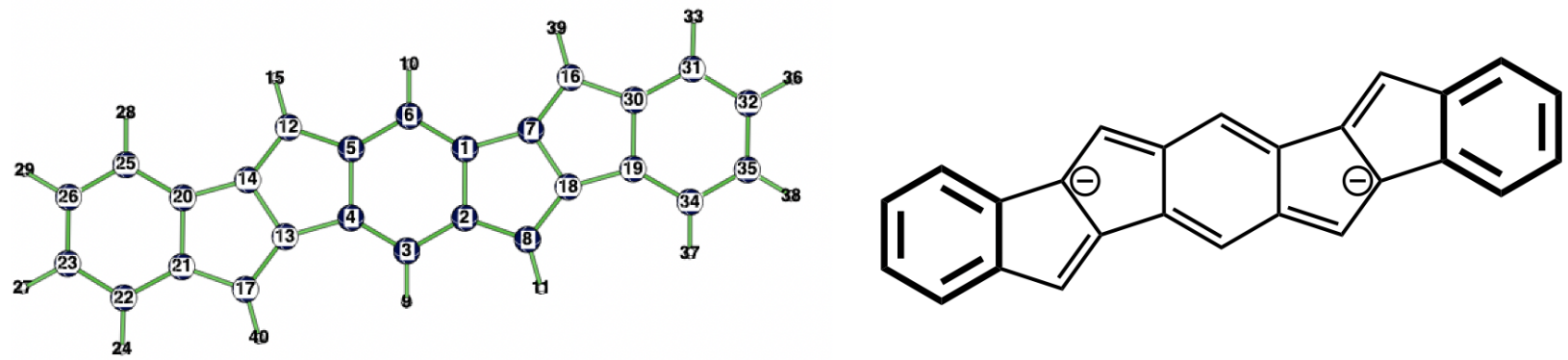

LONE 141181 END

BOND S 12 D $16 \mathrm{~S} 17 \mathrm{~S} 23 \mathrm{D} 28 \mathrm{D} 34 \mathrm{~S} 39 \mathrm{~S} 45 \mathrm{~S} 413 \mathrm{~S} 56 \mathrm{D} 512 \mathrm{~S} 610$

D $716 \mathrm{~S} 718 \mathrm{~S} 811 \mathrm{~S} 818 \mathrm{~S} 1214 \mathrm{~S} 1215 \mathrm{~S} 1314 \mathrm{D} 1317 \mathrm{~S} 1420 \mathrm{~S} 1630$

S 1639 S 1721 S 1740 S 1819 S 1930 D 1934 S 2021 D 2025 D 2122 S 2223

S $2224 \mathrm{D} 2326 \mathrm{~S} 2327 \mathrm{~S} 2526 \mathrm{~S} 2528 \mathrm{~S} 2629 \mathrm{D} 3031 \mathrm{~S} 3132 \mathrm{~S} 3133 \mathrm{D} 3235$

S 3236 S 3435 S 3437 S 3538 END

Figure S25. NLS output string, atom numbering, and Lewis structure for the isolectronic dianion hydrocarbon reference of anti-IDBT 1. Note two Clar sextets in the NLS-recognized Lewis structure.

\section{Dianion hydrocarbon reference for syn-IDBT}

Zero-point correction $=$

Thermal correction to Energy=

Thermal correction to Enthalpy=

Thermal correction to Gibbs Free Energy=

Sum of electronic and zero-point Energies $=$

Sum of electronic and thermal Energies=

Sum of electronic and thermal Enthalpies=

Sum of electronic and thermal Free Energies=
0.300441 (Hartree/Particle)

0.318324

0.319268

0.255528

$-999.106521$

$-999.088638$

$-999.087694$

$-999.151434$

$\begin{array}{llll}\mathrm{C} & -1.21563 & -0.71236 & 0.00000 \\ \mathrm{C} & 0.08738 & -1.44016 & 0.00000 \\ \mathrm{C} & 1.25475 & -0.72300 & 0.00000 \\ \mathrm{C} & 1.21563 & 0.71236 & 0.00000 \\ \mathrm{C} & -0.08738 & 1.44016 & 0.00000 \\ \mathrm{C} & -1.25475 & 0.72300 & 0.00000 \\ \mathrm{C} & -2.24077 & -1.63873 & 0.00000 \\ \mathrm{C} & -1.66505 & -2.97157 & 0.00000 \\ \mathrm{C} & -0.24335 & -2.85115 & 0.00000 \\ \mathrm{H} & 2.21961 & -1.22511 & 0.00000 \\ \mathrm{H} & -2.21961 & 1.22511 & 0.00000\end{array}$




$\begin{array}{llll}\mathrm{H} & -3.29874 & -1.39651 & 0.00000 \\ \mathrm{C} & 0.24335 & 2.85115 & 0.00000 \\ \mathrm{C} & 2.24077 & 1.63873 & 0.00000 \\ \mathrm{C} & 1.66505 & 2.97157 & 0.00000 \\ \mathrm{H} & 3.29874 & 1.39651 & 0.00000 \\ \mathrm{C} & -2.04406 & -4.32378 & 0.00000 \\ \mathrm{C} & 2.04406 & 4.32378 & 0.00000 \\ \mathrm{C} & -0.30257 & 4.15775 & 0.00000 \\ \mathrm{C} & 0.30257 & -4.15775 & 0.00000 \\ \mathrm{C} & 0.83646 & 5.09040 & 0.00000 \\ \mathrm{C} & 0.59496 & 6.47253 & 0.00000 \\ \mathrm{C} & -0.71222 & 6.96357 & 0.00000 \\ \mathrm{H} & 1.43374 & 7.16788 & 0.00000 \\ \mathrm{C} & -1.60801 & 4.68994 & 0.00000 \\ \mathrm{C} & -1.80641 & 6.06930 & 0.00000 \\ \mathrm{H} & -0.89115 & 8.03619 & 0.00000 \\ \mathrm{H} & -2.46615 & 4.02267 & 0.00000 \\ \mathrm{H} & -2.81977 & 6.46520 & 0.00000 \\ \mathrm{C} & -0.83646 & -5.09040 & 0.00000 \\ \mathrm{C} & -0.59496 & -6.47253 & 0.00000 \\ \mathrm{C} & 1.60801 & -4.68994 & 0.00000 \\ \mathrm{C} & 0.71222 & -6.96357 & 0.00000 \\ \mathrm{H} & -1.43374 & -7.16788 & 0.00000 \\ \mathrm{C} & 1.80641 & -6.06930 & 0.00000 \\ \mathrm{H} & 2.46615 & -4.02267 & 0.00000 \\ \mathrm{H} & 0.89115 & -8.03619 & 0.00000 \\ \mathrm{H} & 2.81977 & -6.46520 & 0.00000 \\ \mathrm{H} & -3.04957 & -4.72936 & 0.00000 \\ \mathrm{H} & 3.04957 & 4.72936 & 0.00000\end{array}$

Dianion hydrocarbon reference for anti-IDBT

Zero-point correction $=$

Thermal correction to Energy=

Thermal correction to Enthalpy=

Thermal correction to Gibbs Free Energy=

Sum of electronic and zero-point Energies $=$

Sum of electronic and thermal Energies=

Sum of electronic and thermal Enthalpies=

Sum of electronic and thermal Free Energies=
0.300573 (Hartree/Particle)

0.318394

0.319338

0.255827

$-999.116178$

$-999.098357$

$-999.097413$

$-999.160924$ 


\begin{tabular}{|c|c|c|c|}
\hline $\mathrm{C}$ & 1.32434 & -0.50996 & 0.00000 \\
\hline $\mathrm{C}$ & 1.05838 & 0.93491 & 0.00000 \\
\hline $\mathrm{C}$ & -0.27567 & 1.40840 & 0.00000 \\
\hline $\mathrm{C}$ & -1.32434 & 0.50996 & 0.00000 \\
\hline $\mathrm{C}$ & -1.05838 & -0.93491 & 0.00000 \\
\hline $\mathrm{C}$ & 0.27567 & -1.40840 & 0.00000 \\
\hline $\mathrm{C}$ & 2.77145 & -0.65617 & 0.00000 \\
\hline $\mathrm{C}$ & 2.29366 & 1.62068 & 0.00000 \\
\hline $\mathrm{H}$ & -0.46731 & 2.48072 & 0.00000 \\
\hline $\mathrm{H}$ & 0.46731 & -2.48072 & 0.00000 \\
\hline $\mathrm{H}$ & 2.39749 & 2.70122 & 0.00000 \\
\hline $\mathrm{C}$ & -2.29366 & -1.62068 & 0.00000 \\
\hline $\mathrm{C}$ & -2.77145 & 0.65617 & 0.00000 \\
\hline $\mathrm{C}$ & -3.34159 & -0.67087 & 0.00000 \\
\hline $\mathrm{H}$ & -2.39749 & -2.70122 & 0.00000 \\
\hline $\mathrm{C}$ & 3.79684 & -1.60948 & 0.00000 \\
\hline $\mathrm{C}$ & -3.79684 & 1.60948 & 0.00000 \\
\hline $\mathrm{C}$ & 3.34159 & 0.67087 & 0.00000 \\
\hline $\mathrm{C}$ & 4.76641 & 0.53792 & 0.00000 \\
\hline $\mathrm{C}$ & -4.76641 & -0.53792 & 0.00000 \\
\hline $\mathrm{C}$ & -5.04347 & 0.90003 & 0.00000 \\
\hline $\mathrm{C}$ & -6.37908 & 1.33356 & 0.00000 \\
\hline $\mathrm{C}$ & -7.42410 & 0.40615 & 0.00000 \\
\hline $\mathrm{H}$ & -6.60373 & 2.39914 & 0.00000 \\
\hline $\mathrm{C}$ & -5.83561 & -1.44203 & 0.00000 \\
\hline $\mathrm{C}$ & -7.15511 & -0.97809 & 0.00000 \\
\hline $\mathrm{H}$ & -8.45473 & 0.75410 & 0.00000 \\
\hline $\mathrm{H}$ & -5.64163 & -2.51281 & 0.00000 \\
\hline $\mathrm{H}$ & -7.97879 & -1.68805 & 0.00000 \\
\hline $\mathrm{C}$ & 5.04347 & -0.90003 & 0.00000 \\
\hline $\mathrm{C}$ & 6.37908 & -1.33356 & 0.00000 \\
\hline $\mathrm{C}$ & 7.42410 & -0.40615 & 0.00000 \\
\hline $\mathrm{H}$ & 6.60373 & -2.39914 & 0.00000 \\
\hline $\mathrm{C}$ & 5.83561 & 1.44203 & 0.00000 \\
\hline $\mathrm{C}$ & 7.15511 & 0.97809 & 0.00000 \\
\hline $\mathrm{H}$ & 8.45473 & -0.75410 & 0.00000 \\
\hline $\mathrm{H}$ & 5.64163 & 2.51281 & 0.00000 \\
\hline $\mathrm{H}$ & 7.97879 & 1.68805 & 0.00000 \\
\hline $\mathrm{H}$ & 3.69514 & -2.68852 & 0.00000 \\
\hline $\mathrm{H}$ & -3.69514 & 2.68852 & 0.00000 \\
\hline
\end{tabular}




\subsection{Charge-Effects in IDT and IDT-S Fused $s$-Indacenes}

When the two terminal benzene rings of IDBT and IDBT-S are removed, both thiophene (IDT) and thiophene-dioxide (IDT-S) fused $s$-indacenes exhibit a more paratropic anti-isomer. In these systems, the "Clar sextet-effect," arising from differences in the dominant resonance forms of the outer benzenoid rings is absent, and a "charge-effect" (i.e., topological charge stabilization) dominates. Computed NPA charges for the $s$-indacene core show that carbons 2 and 6 display the most negative charges (see Figure 4 in the main text). In the syn-forms, the $\mathrm{S}$ and $\mathrm{SO}_{2}$ groups stabilize negative charges at carbons 2 and 6, resulting in larger HOMO-LUMO gaps (S: 2.29 eV, $\mathrm{SO}_{2}: 2.27 \mathrm{eV}$ ) and decreased paratropicity. In the anti-forms, the $\mathrm{S}$ and $\mathrm{SO}_{2}$ groups destabilize charges at carbons 2 and 6, resulting in smaller HOMO-LUMO gaps ( $\left.\mathrm{S}: 1.35 \mathrm{eV}, \mathrm{SO}_{2}: 1.28 \mathrm{eV}\right)$ and increased paratropicity.

Geometries for IDT and IDT-S were optimized at B3LYP/6-311++G(d,p) using the Gaussian16 program. ${ }^{10}$ Optimized Cartesian coordinates are included in Section 7.1 of the SI. Natural Population Analyses (NPA) analyses were performed at the same level of theory using the NBO 7.0 program $^{11}$ as implemented in Gaussian16. HOMO-LUMO gaps were computed as vertical excitation energies from the HOMO to the LUMO orbitals at TD-B3LYP/6-311++G(d,p).
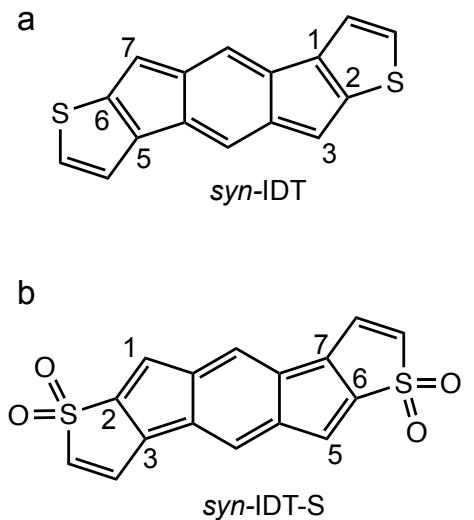
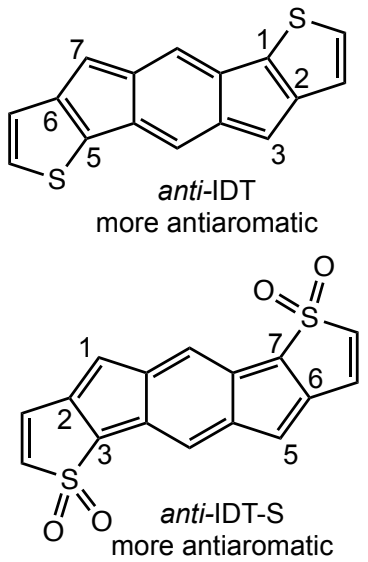

Figure S26. Structures for (a) IDT (shown in "normal" bonding pattern) and (b) IDT-S (shown in "flipped" bonding pattern). Both systems show a more paratropic anti- form, as expected by a "charge-effect" (i.e., topological charge stabilization; see Figure S25). Electronegative groups stabilize the $s$-indacene core when attached to positions 2 and $6(s y n-)$, and destabilize the $s$ indacene core when attached to positions 1 and 5 or 3 and 7 (anti-). 


\subsection{Optimized Geometries and Calculated $y$ and $\Delta \mathbf{E}_{S T}$ Values}

Geometry optimization and frequency analysis calculations for the singlet and triplet states were performed at the RB3LYP and UB3LYP levels, respectively, using the $6-311 \mathrm{G}^{*}$ basis set. Vertical and adiabatic $\Delta E_{\mathrm{ST}}$ values were evaluated at the SF-NC-TDDFT PBE50/6-311G* level, ${ }^{12}$ where zero-point vibrational energy (ZPVE) corrections for the singlet and triplet states were estimated from the results of the frequency analysis calculations at the RB3LYP and UB3LYP levels, respectively. Diradical character $y$ for the singlet state was evaluated at the PUHF/6-311G* level. Quantum chemical calculations were performed using Gaussian 09 ${ }^{13}$ and Q-Chem $5^{14}$ program packages. Calculation results were visualized using DrawMol package. ${ }^{15}$

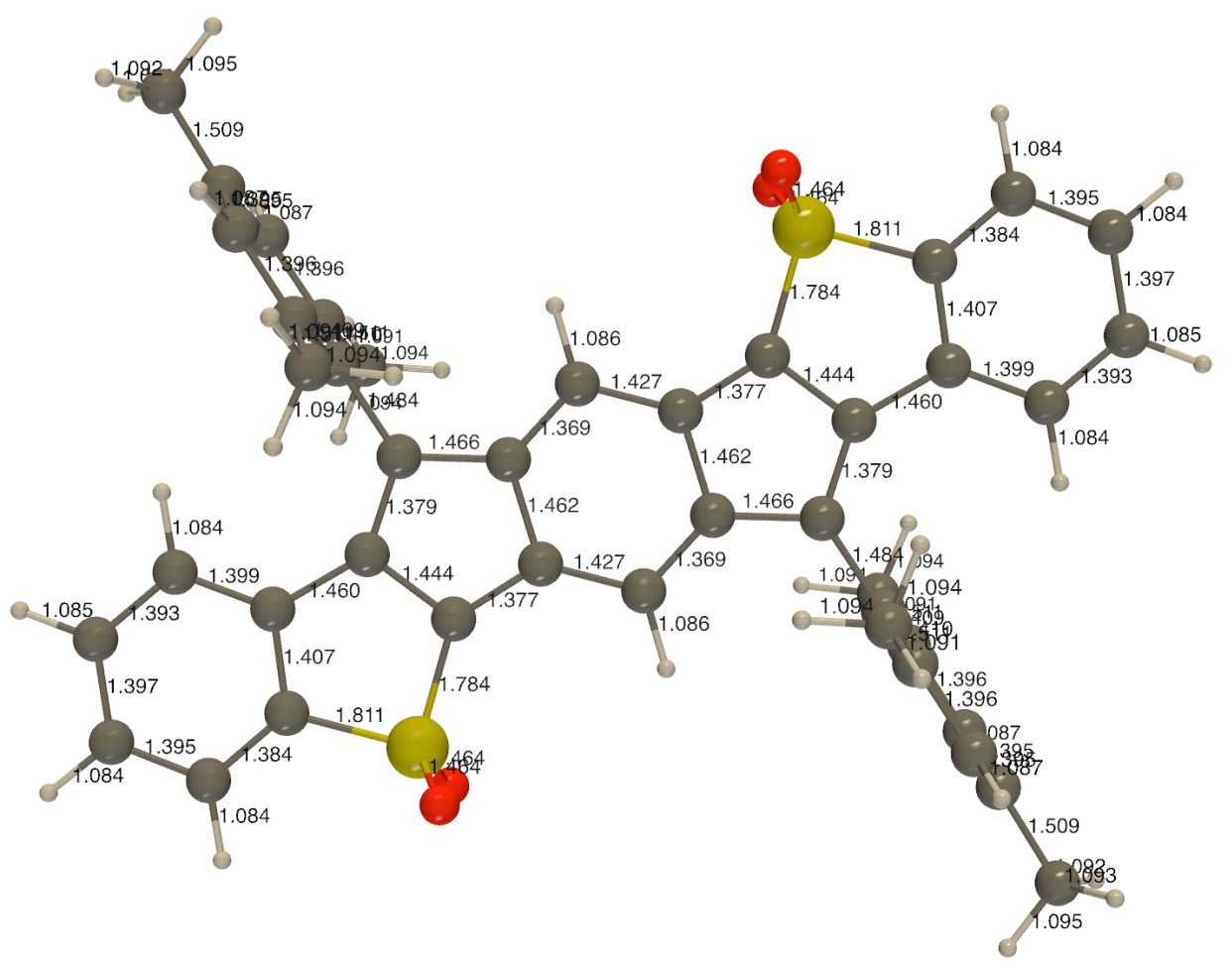

Figure S27. Optimized geometry of anti-IDBT-sulfone 5. 


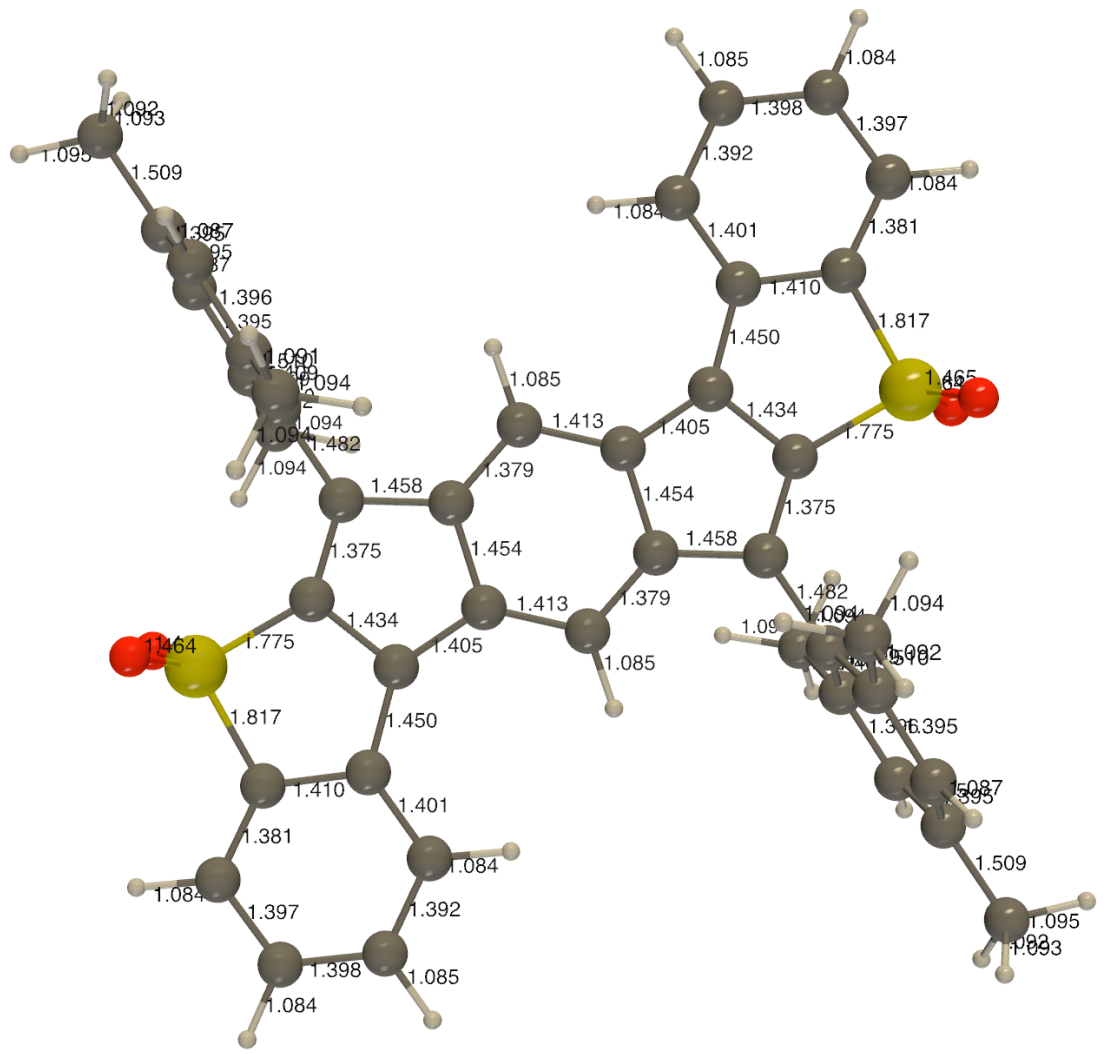

Figure S28. Optimized geometry of syn-IDBT-sulfone $\mathbf{6}$.

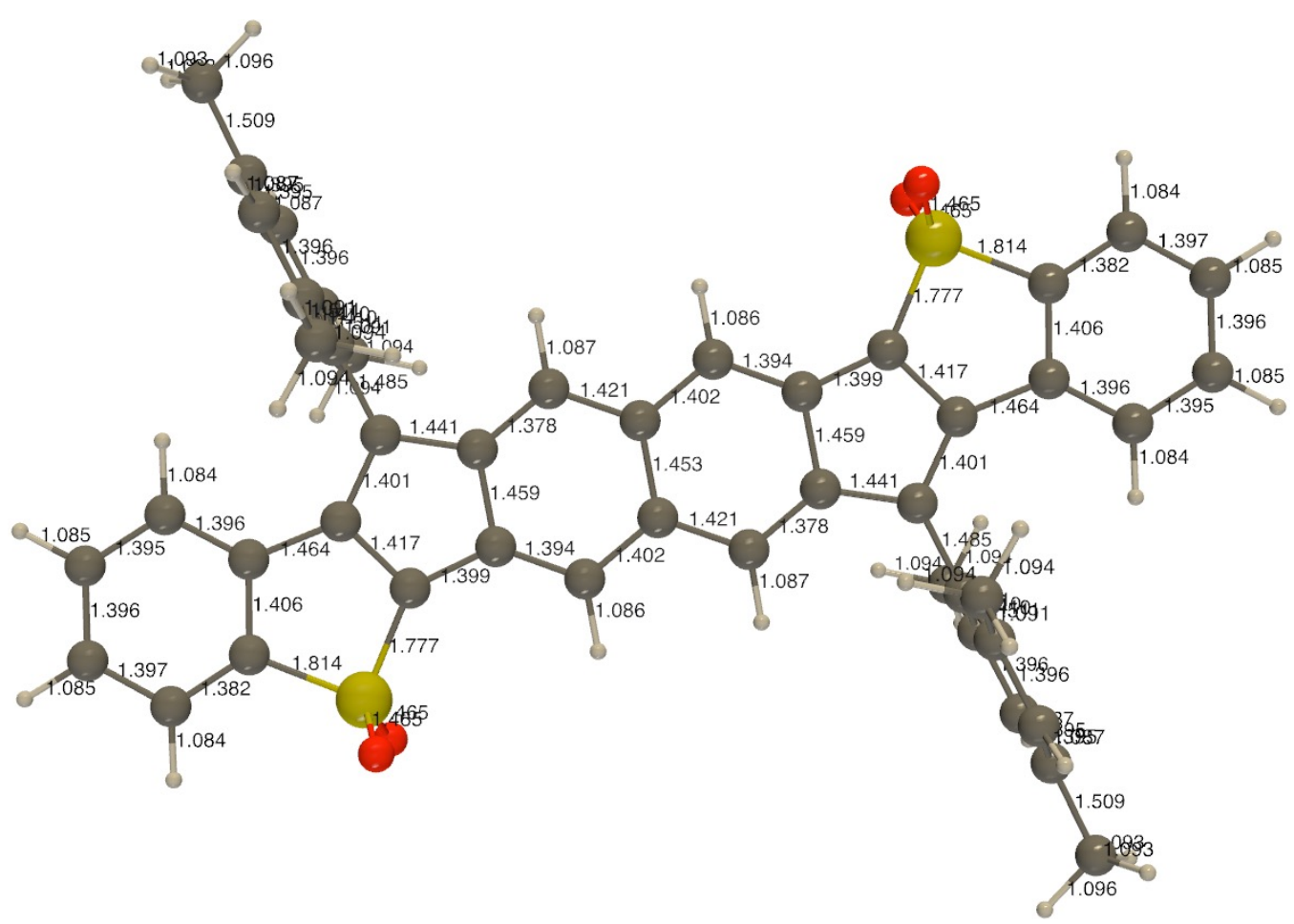

Figure S29. Optimized geometry of anti-IIDBT-sulfone 7. 


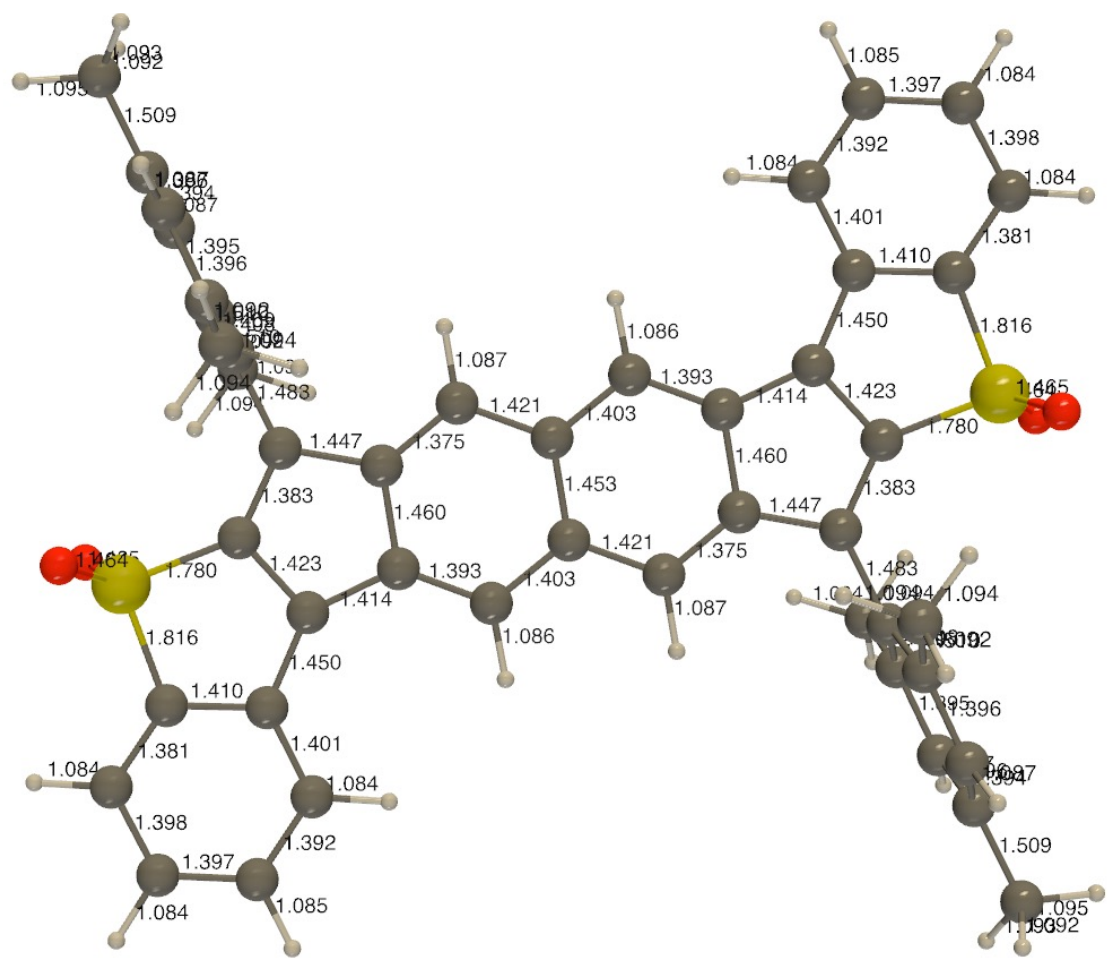

Figure S30. Optimized geometry of syn-IIDBT-sulfone 8.

Table S2. Summary of calculation results.

\begin{tabular}{lcccc}
\hline & \multicolumn{2}{c}{ IIDBT } & \multicolumn{2}{c}{ IIDBT-sulfone } \\
\cline { 2 - 5 } & anti (3) & syn (4) & anti (7) & syn $(\mathbf{8})$ \\
\hline$y$ (PUHF) $[-]$ & 0.613 & 0.658 & 0.601 & 0.652 \\
Vert. $\Delta E_{\text {ST }}\left(\mathrm{kcal} \mathrm{mol}^{-1}\right)$ & -11.65 & -11.09 & -13.59 & -11.26 \\
Adia. $\Delta E_{\text {ST }}\left(\mathrm{kcal} \mathrm{mol}^{-1}\right)$ & -9.37 & -8.84 & -10.72 & -9.33 \\
Adia. $\Delta E_{\text {ST }}\left(+\mathrm{ZPVE}\left(\mathrm{kcal} \mathrm{mol}^{-1}\right)\right.$ & -8.77 & -8.06 & -9.65 & -8.29 \\
$\Delta E_{\text {ST }}(\exp )\left(\mathrm{kcal} \mathrm{mol}^{-1}\right)$ & -8.3 & -7.1 & n.a. & -6.5 \\
\hline
\end{tabular}




\section{SQUID Details and TGA Analysis}

Magnetic susceptibility measurements were performed with a Quantum Design MPMS-XL5 SQUID susceptometer in the 300-580 K temperature range with an applied field of $0.5 \mathrm{~T}$ using a sample space oven with the sample $(\mathrm{m}=4.528 \mathrm{mg})$ inserted in a $1.5 \mathrm{~mm}$ diameter aluminum foil cylinder. The susceptibility data were corrected for the same sample holder previously measured using the same conditions and for the diamagnetic contributions of the compound as deduced by using Pascal's constant tables. ${ }^{16}$ The magnetic measurements were fitted using the classical Bleaney-Bowers model for an antiferromagentic $S=1 / 2$ dimer plus a monomeric $S=1 / 2$ impurity. ${ }^{17}$

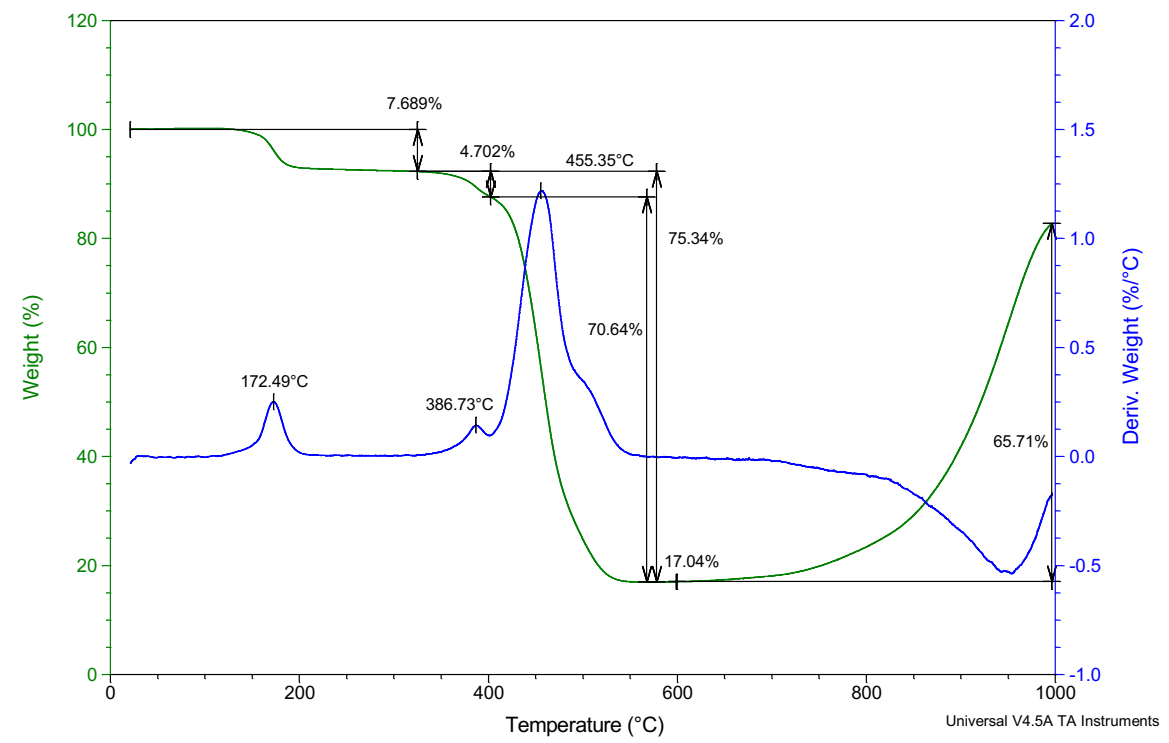

Figure S31. TGA data for syn-IIDBT-S 8. Weight loss at $\sim 170{ }^{\circ} \mathrm{C}$ corresponds to loss of residual 1,2-dichlorobenzene solvent (or possibly loss of a $t$-butyl group). 


\section{References}

1. Sheldrick, G. M. Bruker/Siemens Area Detector Absorption Correction Program, Bruker AXS, Madison, WI, 1998.

2. van der Sluis, P.; Spek, A. L. Acta Cryst., Sect. A 1990, A46, 194-201.

3. Sheldrick, G. M. Acta Cryst. 2015, C71, 3-8.

4. CrysAlisPro; Rigaku OD, The Woodlands, TX, 2015.

5. Sheldrick, G. M. Acta Cryst. 2008, A64, 112-122.

6. Müller, P. Crystallogr. Rev. 2009, 15, 57-83.

7. Frisch, M. J.; Trucks, G. W.; Schlegel, H. B.; Scuseria, G. E.; Robb, M. A.; Cheeseman, J. R.; Scalmani, G.; Barone, V.; Mennucci, B.; Petersson, G. A.; Nakatsuji, H.; Caricato, M.; Li, X.; Hratchian, H. P.; Izmaylov, A. F.; Bloino, J.; Zheng, G.; Sonnenberg, J. L.; Hada, M.; Ehara, M.; Toyota, K.; Fukuda, R.; Hasegawa, J.; Ishida, M.; Nakajima, T.; Honda, Y.; Kitao, O.; Nakai, H.; Vreven, T.; Montgomery, Jr., J. A.; Peralta, J. E.; Ogliaro, F.; Bearpark, M.; Heyd, J. J.; Brothers, E.; Kudin, K. N.; Staroverov, V. N.; Keith, T.; Kobayashi, R.; Normand, J.; Raghavachari, K.; Rendell, A.; Burant, J. C.; Iyengar, S. S.; Tomasi, J.; Cossi, M.; Rega, N.; Millam, J. M.; Klene, M.; Knox, J. E.; Cross, J. B.; Bakken, V.; Adamo, C.; Jaramillo, J.; Gomperts, R.; Stratmann, R. E.; Yazyev, O.; Austin, A. J.; Cammi, R.; Pomelli, C.; Ochterski, J. W.; Martin, R. L.; Morokuma, K.; Zakrzewski, V. G.; Voth, G. A.; Salvador, P.; Dannenberg, J. J.; Dapprich, S.; Daniels, A. D.; Farkas, O.; Foresman, J. B.; Ortiz, J. V.; Cioslowski, J.; and Fox, D. J. Gaussian 09, Revision E.01; Gaussian, Inc.: Wallingford CT, 2013.

\section{Rahalkar, A.; Stanger, A. http://schulich.technion.ac.il/Amnon_Stanger.htm}

9. (a) Stanger, A. J. Org. Chem. 2006, 71, 883-893; (b) Stanger, A. J. Org. Chem. 2010, 75, 22812288; (c) Gershoni-Poranne, R.; Stanger, A. Chem.-Eur. J. 2014, 20, 5673-5688.

10. Frisch, M. J., Trucks, G. W., Schlegel, H. B., Scuseria, G. E., Robb, M. A., Cheeseman, J. R., Scalmani, G., Barone, V., Petersson, G. A., Nakatsuji, H., Li, X., Caricato, M., Marenich, A. V., Bloino, J., Janesko, B. G., Gomperts, R., Mennucci, B., Hratchian, H. P., Ortiz, J. V., Izmaylov, A. F., Sonnenberg, J. L., Williams-Young, D., Ding, F., Lipparini, F., Egidi, F., Goings, J., Peng, B., Petrone, A., Henderson, T., Ranasinghe, D., Zakrzewski, V. G., Gao, J., Rega, N., Zheng, G., Liang, W., Hada, M., Ehara, M., Toyota, K., Fukuda, R., Hasegawa, J., Ishida, M., Nakajima, T., Honda, Y., Kitao, O., Nakai, H., Vreven, T., Throssell, K., Montgomery, J. A., Jr., Peralta, J. E., Ogliaro, F., Bearpark, M. J., Heyd, J. J., Brothers, E. N., Kudin, K. N., Staroverov, V. N., Keith, T. A., Kobayashi, R., Normand, J., Raghavachari, K., Rendell, A. P., Burant, J. C., Iyengar, S. S., Tomasi, J., Cossi, M., Millam, J. M., Klene, M., Adamo, C., Cammi, R., Ochterski, J. W., Martin, R. L., Morokuma, K., Farkas, O., Foresman, J. B., Fox, D. J. Gaussian 16, Revision C.01; Gaussian, Inc.: Wallingford CT, 2016. 
11. Glendening, E. D.; Badenhoop, J. K.; Reed, A. E.; Carpenter, J. E.; Bohmann, J. A.; Morales, C. M.; Karafiloglou, P.; Landis, C. R.; Weinhold, F. NBO 7.0, Theoretical Chemistry Institute, University of Wisconsin, Madison, 2018.

12. (a) Shao, Y.; Head-Gordon, M.; Krylov, A. I. J. Chem. Phys. 2003, 118, 4807-4818. (b) Wang, F.; Ziegler, T. J. Chem. Phys. 2004, 121, 12191-12196. (c) Wang, F.; Ziegler, T. J. Chem. Phys. 2005, 122, 074109-1-9. c) Wang, F.; Ziegler, T. Int. J. Quantum Chem. 2006, 106, 2545-2550.

13. Frisch, M. J.; Trucks, G. W.; Schlegel, H. B.; Scuseria, G. E.; Robb, M. A.; Cheeseman, J. R.; Scalmani, G.; Barone, V.; Mennucci, B.; Petersson, G. A.; Nakatsuji, H.; Caricato, M.; Li, X.; Hratchian, H. P.; Izmaylov, A. F.; Bloino, J.; Zheng, G.; Sonnenberg, J. L.; Hada, M.; Ehara, M.; Toyota, K.; Fukuda, R.; Hasegawa, J.; Ishida, M.; Nakajima, T.; Honda, Y.; Kitao, O.; Nakai, H.; Vreven, T.; Montgomery, J. A., J.; Peralta, J. E.; Ogliaro, F.; Bearpark, M.; Heyd, J. J.; Brothers, E.; Kudin, K. N.; Staroverov, V. N.; Kobayashi, R.; Normand, J.; Raghavachari, K.; Rendell, A.; Burant, J. C.; Iyengar, S. S.; Tomasi, J.; Cossi, M.; Rega, N.; Millam, J. M.; Klene, M.; Knox, J. E.; Cross, J. B.; Bakken, V.; Adamo, C.; Jaramillo, J.; Gomperts, R.; Stratmann, R. E.; Yazyev, O.; Austin, A. J.; Cammi, R.; Pomelli, C.; Ochterski, J. W.; Martin, R. L.; Morokuma, K.; Zakrzewski, V. G.; Voth, G. A.; Salvador, P.; Dannenberg, J. J.; Dapprich, S.; Daniels, A. D.; Farkas, Ö.; Foresman, J. B.; Ortiz, J. V.; Cioslowski, J.; Fox, D. J. Gaussian 09, Revision D.01; Gaussian, Inc.: Wallingford CT, 2009.

14. Shao, Y.; Gan, Z.; Epifanovsky, E.; Gilbert, A. T. B.; Wormit, M.; Kussmann, J.; Lange, A. W.; Behn, A.; Deng, J.; Feng, X.; Ghosh, D.; Goldey, M.; Horn, P. R.; Jacobson, L. D.; Kaliman, I.; Khaliullin, R. Z.; Kuś, T.; Landau, A.; Liu, J.; Proynov, E. I.; Rhee, Y. M.; Richard, R. M.; Rohrdanz, M. A.; Steele, R. P.; Sundstrom, E. J.; Woodcock, H. L.; Zimmerman, P. M.; Zuev, D.; Albrecht, B.; Alguire, E.; Austin, B.; Beran, G. J. O.; Bernard, Y. A.; Berquist, E.; Brandhorst, K.; Bravaya, K. B.; Brown, S. T.; Casanova, D.; Chang, C.-M.; Chen, Y.; Chien, S. H.; Closser, K. D.; Crittenden, D. L.; Diedenhofen, M.; DiStasio, R. A.; Do, H.; Dutoi, A. D.; Edgar, R. G.; Fatehi, S.; Fusti-Molnar, L.; Ghysels, A.; Golubeva-Zadorozhnaya, A.; Gomes, J.; Hanson-Heine, M. W. D.; Harbach, P.H.P.; Hauser, A. W. ; Hohenstein, E. G.; Holden, Z. C.; Jagau, T.-C.; Ji, H.; Kaduk, B.n; Khistyaev, K.; Kim, J.; Kim, J.; King, R. A.; Klunzinger, P.; Kosenkov, D.; Kowalczyk, T.; Krauter, C. M.; Lao, K. U.; Laurent, A. D.; Lawler, K. V.; Levchenko, S. V.; Lin, C. Y.; Liu, F.; Livshits, E.; Lochan, R. C.; Luenser, A.; Manohar, P.; Manzer, S. F.; Mao, S.-P.; Mardirossian, N.; Marenich, A. V.; Maurer, S. A.; Mayhall, N. J.; Neuscamman, E.; Oana, C. M.; OlivaresAmaya, R.; O’Neill, D. P.; Parkhill, J. A.; Perrine, T. M.; Peverati, R.; Prociuk, A.; Rehn, D. R.; Rosta, E.; Russ, N. J.; Sharada, S. M.; Sharma, S.; Small, D. W.; Sodt, A.; Stein, T.; Stück, D.; Su, Y.-C.; Thom, A. J. W.; Tsuchimochi, T.; Vanovschi, V.; Vogt, L.; Vydrov, O.; Wang, T.; Watson, M. A.; Wenzel, J.; White, A.; Williams, C. F.; Yang, J.; Yeganeh, S.; Yost, S. R.; You, Z.-Q.; Zhang, I. Y.; Zhang, X.; Zhao, Y.; Brooks, B. R.; Chan, G. K. L.; Chipman, D. M.; Cramer, C. J.; Goddard, W. A.; Gordon, M. S.; Hehre, W. J.; Klamt, A.; Schaefer, H. F.; Schmidt, M. W.; Sherrill, C. D.; Truhlar, D. G.; Warshel, A.; Xu, X.; Aspuru-Guzik, A.; Baer, R.; Bell, A. T.; Besley, N. A.; Chai, J.-D.; Dreuw, A.; Dunietz, B. D.; Furlani, T. R.; Gwaltney, S. R.; Hsu, C.-P.; Jung, Y.; Kong, J.; Lambrecht, D. S.; Liang, W.; Ochsenfeld, C.; Rassolov, V. A.; Slipchenko, L.V.; Subotnik, J. E.; Voorhis, T. V.; Herbert, J. M.; Krylov, A. I.; Gill, P. M.W.; Head-Gordon, M. Mol. Phys. 2015, $113,184-215$. 
15. Liegeois, V. DrawMol ver. 1.5, UNamur, www.unamur.be/drawmol.

16. Bain, G. A.; Berry, J. F. J. Chem. Educ. 2008, 85, 532-536.

17. Bleaney, B.; Bowers, K. D. Proc. R. Soc. Lond. A. 1952, 214, 451-465. 